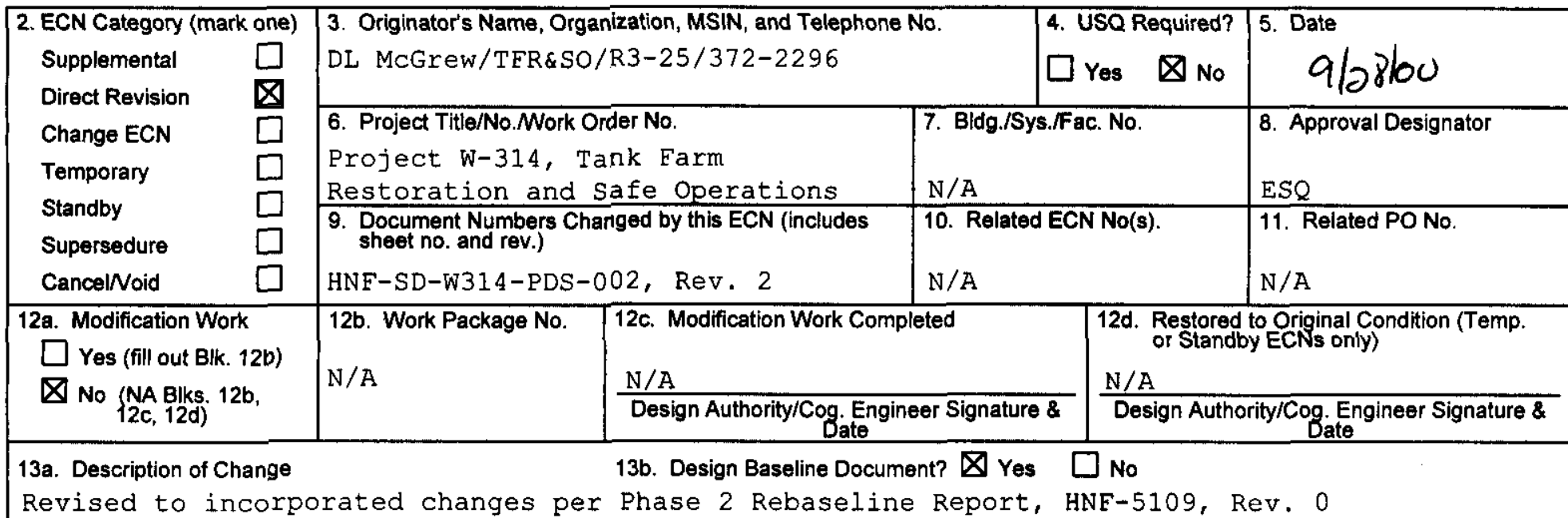

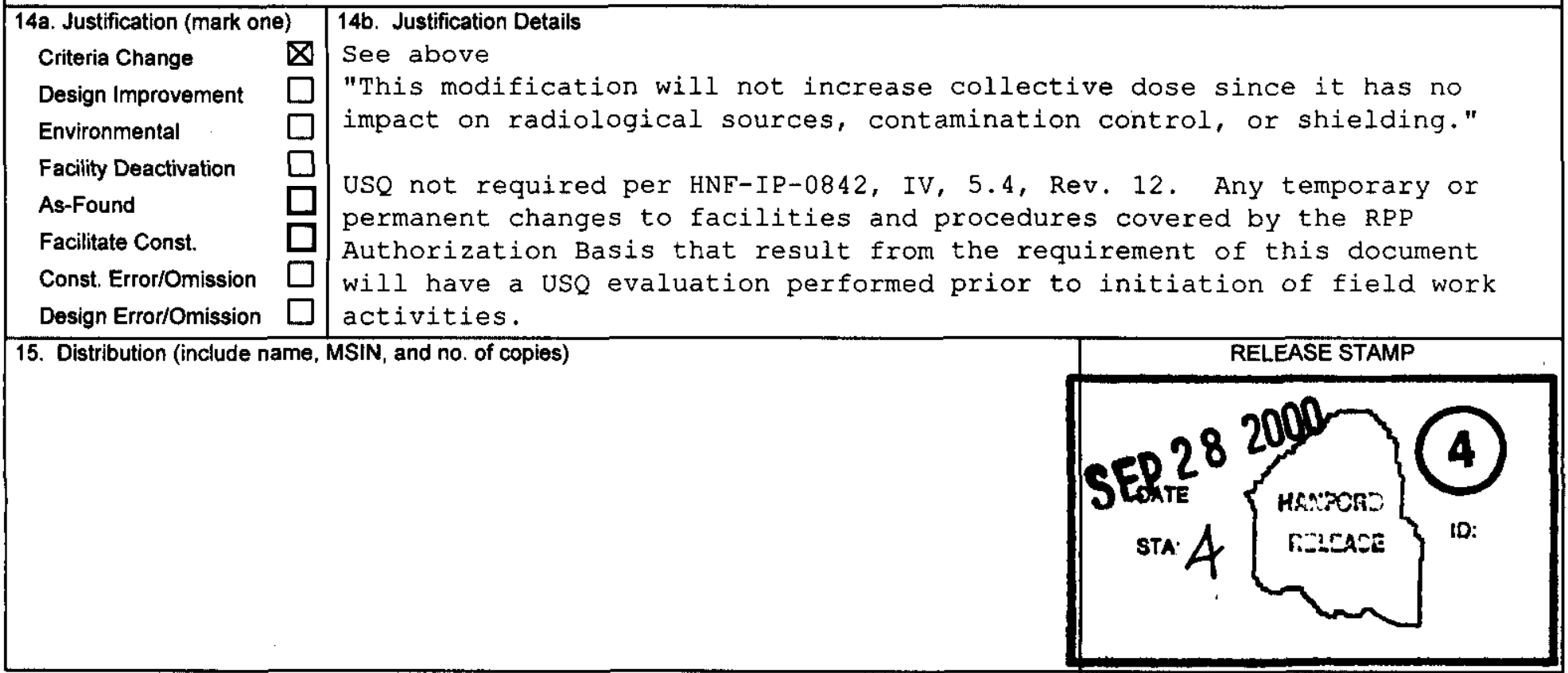




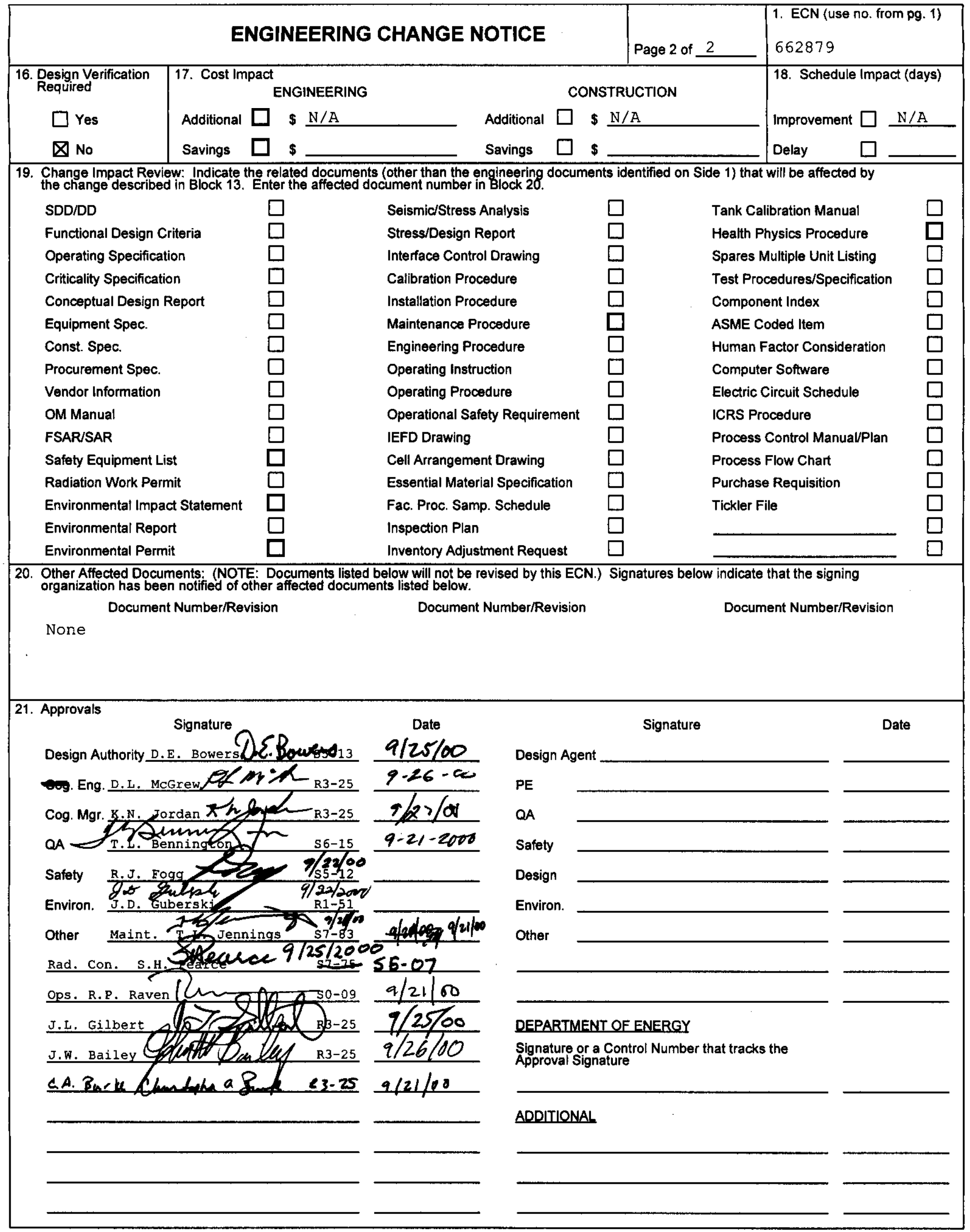




\section{DISTRIBUTION SHEET}

To

Distribution

Project TitleMork Order

W-314, Tank Farm Restoration and Safe Operations

\section{From \\ TER\&SO}

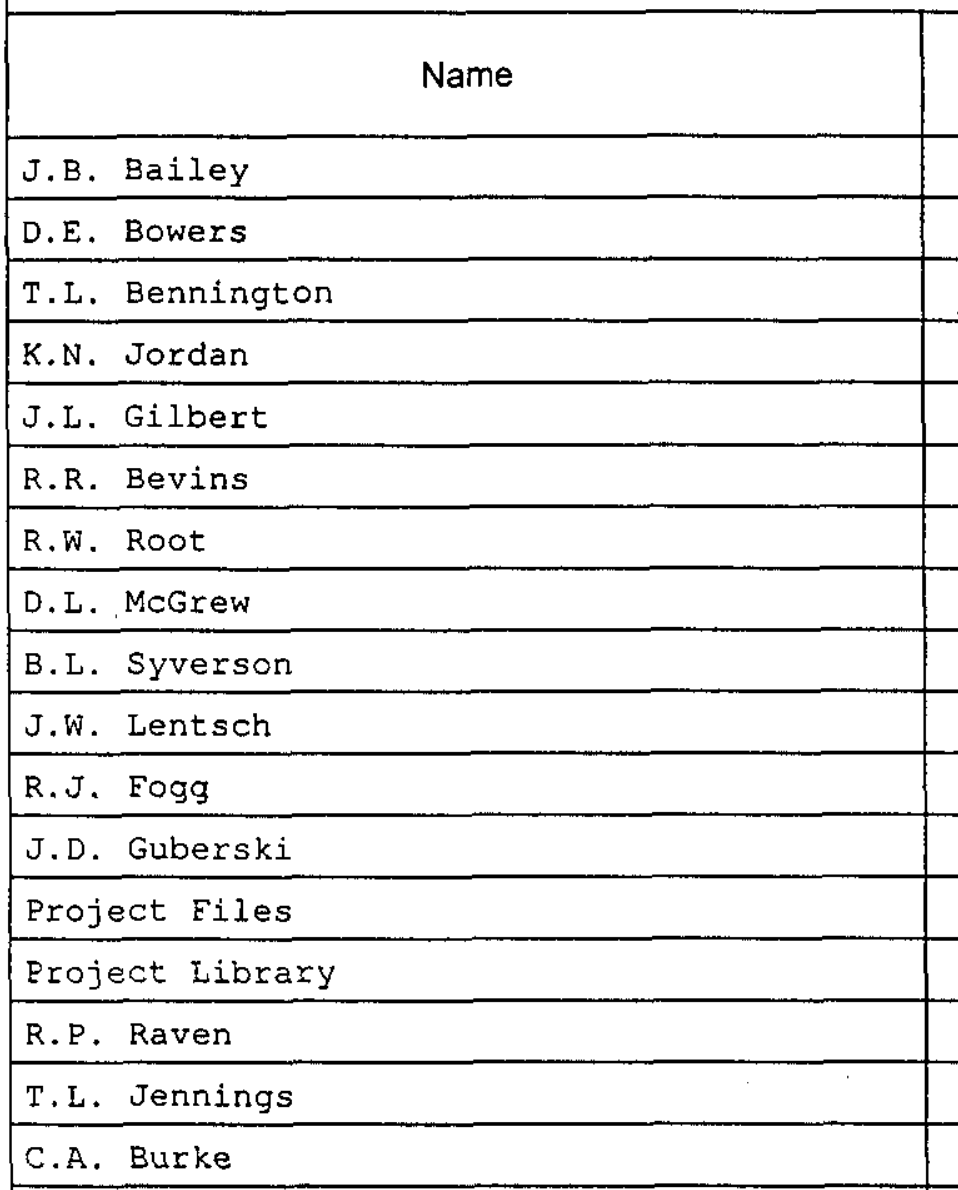

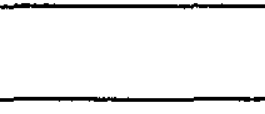

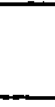

Page 1 of 1

EDT No.

ECN No. 662879

\begin{tabular}{|c|c|c|c|c|}
\hline MSIN & $\begin{array}{c}\text { Text } \\
\text { With All } \\
\text { Attach. }\end{array}$ & Text Only & $\begin{array}{l}\text { Attach.l } \\
\text { Appendix } \\
\text { Only }\end{array}$ & $\begin{array}{c}\text { EDT/ECN } \\
\text { Only }\end{array}$ \\
\hline
\end{tabular}

R3-25

S5-13

R2-89

R3-25

R3-25

R3-25

R3-53

R3-25

G3-12

R3-25

S5-12

R1-51

R1-29

R3-25

So-09

S7-90

R3-25

$x$
$x$
$x$
$x$
$x$
$x$
$x$
$x$
$x$
$x$
$x$
$x$
$x$
$x$
$x$
$x$
$x$




\title{
Project Development Specification for Valve Pit Manifold
}

\author{
DL MCGrew
}

$\mathrm{NHC}$

Richland, WA 99352

U.S. Department of Energy Contract DE-AC06-99RL14047

\author{
EDT/ECN: 662879 UC: \\ Cost Center: 7C900 Charge Code: 109749 \\ B\&R Code: \\ Total Pages: 78
}

Key Words: Project W-314, Tank Earm Restoration and Safe Operations, valve pit manifold

\begin{abstract}
Establishes the performance, design development, and test requirements for the valve pit manifolds. The system engineering approach was used to develop this document in accordance with the guidelines laid out in the Systems Engineering Management Plan for Project $\mathrm{W}-314$.
\end{abstract}

TRADEMARK DISCLAIMER. Reference herein to any specific commercial product, process, or service by trade name, trademark, manufacturer, or otherwise, does not necessarily constitute or imply its endorsement, recommendation, or favoring by the United States Government or any agency thereof or its contractors or subcontractors.

Printed in the United States of America. To obtain copies of this document, contact: Document Control Services, P.O. Box 950, Mailstop H6-08, Richland WA 99352, Phone (509) 372-2420; Fax (509) 376-4989.

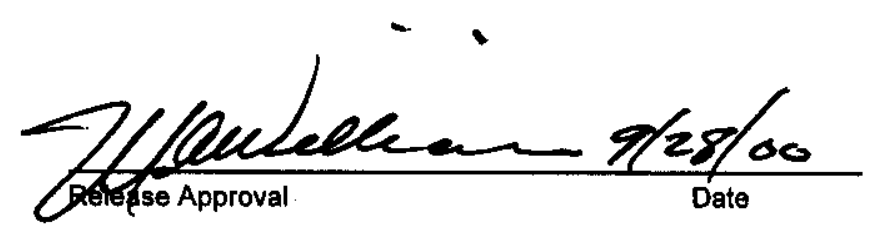

Approved For Public Release 
RECORD OF REVISION

(2) Title

Project Development Specification for Valve Pit Manifold

Change Control Record

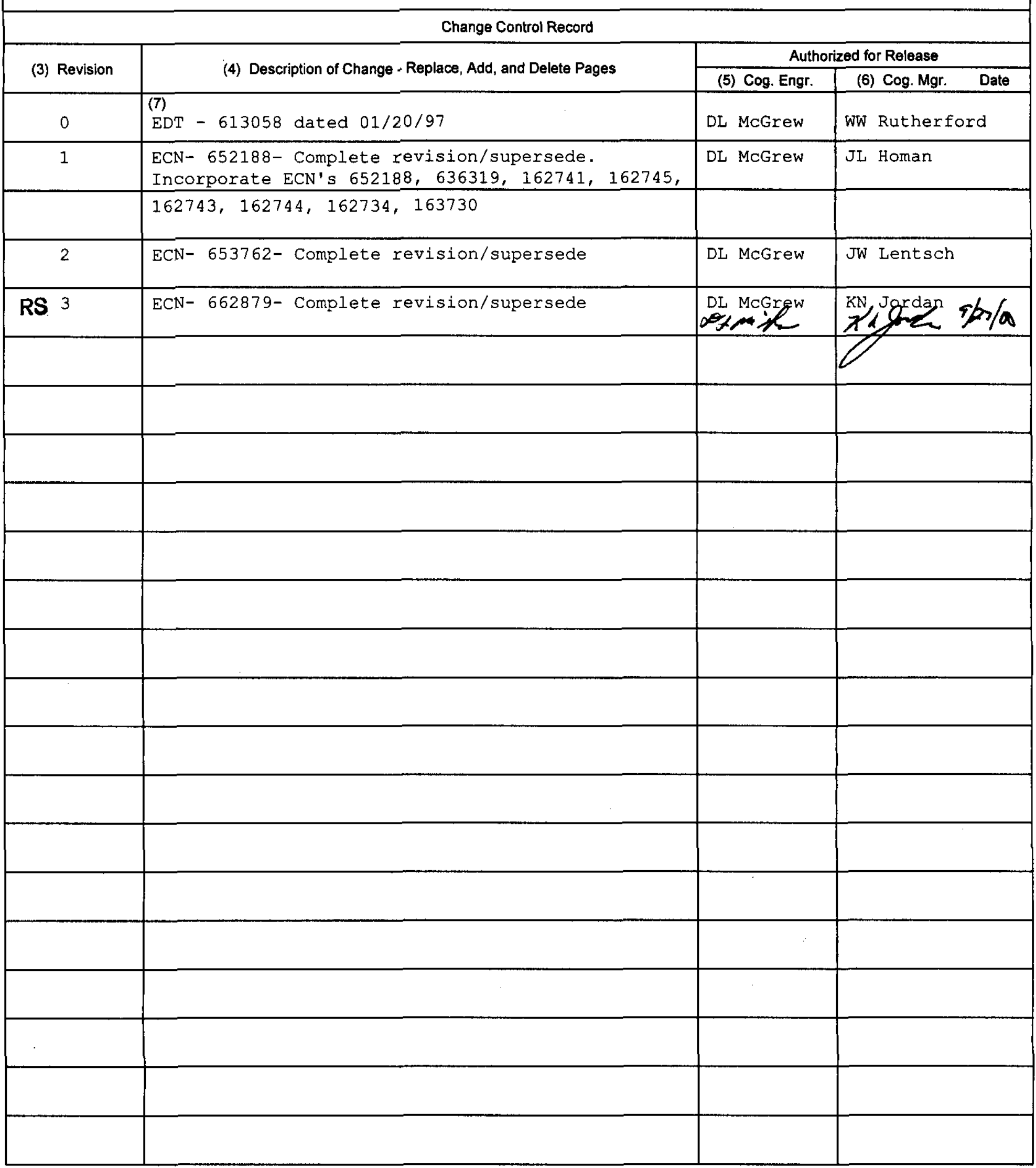




\section{Project Development Specification}

$$
\text { for }
$$

\section{Valve Pit Manifold}

Project W-314

Tank Farm Restoration and Safe Operations

September 2000

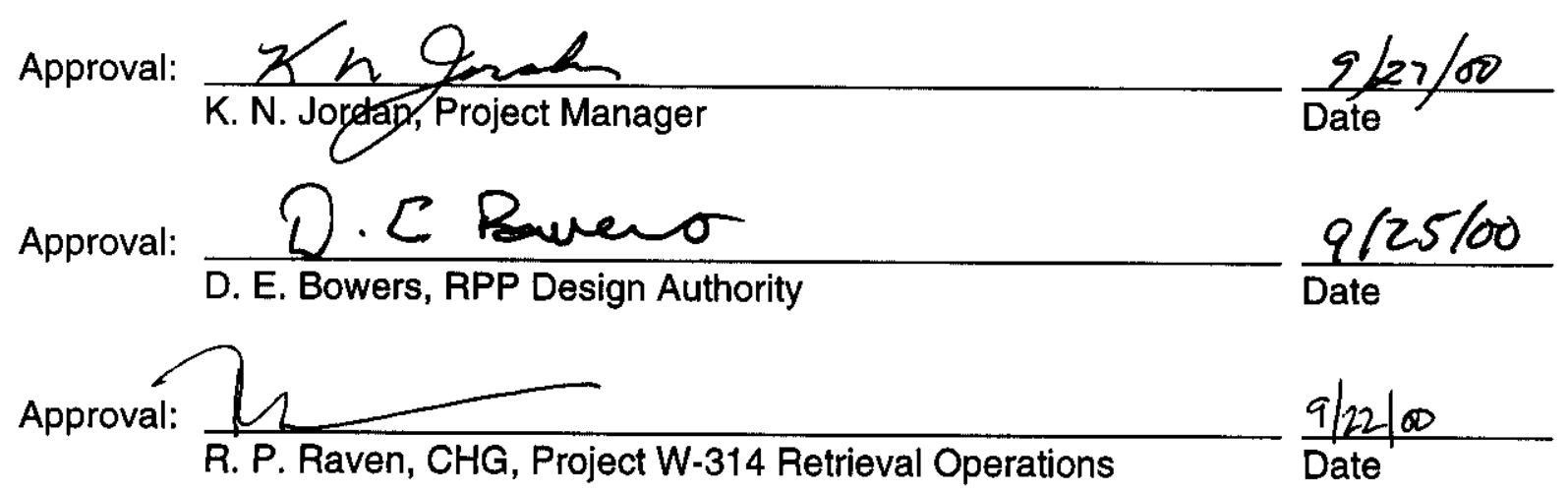




\section{Table of Contents}

1 SCOPE__ 1-1

1.1 Description___ 1-1

1.2 Classification__ 1-1

2 APPLICABLE DOCUMENTS__ 2-1

2.1 DOE Documents____ 2-1

2.2 Code of Federal Regulations____ 2-1

2.3 Tank Farm Contractor Documents__ 2-1

2.4 Project W-314 Documents___ 2-1

2.5 Codes and Standards___ 2-1

2.6 Other Documents________ 2-2

2.7 Drawings___ 2-2

3 REQUIREMENTS____ 3-1

3.1 Item Definition__ 3-1

3.1.1 Item Diagrams___ 3-7

3.1.2 Interface Definition___ 3-8

3.1.3 Major Component List_ 3-8

3.2 Characteristics___ 3-8

3.2.1 Performance Characteristics___ 3-8

3.2.1.1 Confine Waste Within Valve Manifolds and Jumpers____ 3-8

3.2.1.1.1 Corrosion/Erosion___ 3-8

3.2.1.1.2 Reynolds Number___ 3-8

3.2.1.1.3 Head Loss_____ 3-8

3.2.1.1.4 Design Pressure___ 3-8

3.2.1.1.5 WT-SLL-3160 Design Pressure___ 3-9

3.2.1.1.6 WT-SLL-3160 Encasement Design Pressure___ 3-9

3.2.1.1.7 Temperature Maintenance___ 3-9

3.2.1.1.8 Compatibility with Waste___ 3-9

3.2.1.2 Position Manifold Valves___ 3-12

3.2.1.2.1 Remote Valve Operation___ 3-12

3.2.1.3 Confine Flush in Valve Manifolds and Jumpers___ 3-12

3.2.1.3.1 Compatibility with Flush Water___ 3-12

3.2.2 Physical Characteristics___ 3-12

3.2.2.1 Valve Piping System Features___ 3-12

3.2.2.2 Valve Extension Handles___ 3-12

3.2.2.3 PUREX Jumper Connector___ 3-12

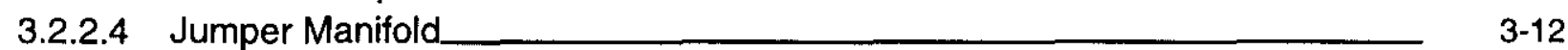

3.2.2.5 Valve Position Indication System____ 3-12

3.2.2.6 Not used___ 3-12

3.2.2.7 Valve Position Indicators___ 3-12

3.2.2.8 Valve Lock Capacity___ 3-12

3.2.3 Reliability__ 3-12 
3.2.3.1 Design Life of Valve Manifold__ 3-13

3.2.3.2 Design Life of Cover Blocks___ 3-13

3.2.3.3 Design Life of Slurry Receiver Pit__ 3-13

3.2.3.4 Degree of Reliability____ 3-13

3.2.3.5 Valve Life___ 3-13

3.2.3.6 Fault Detection/lsolation___ 3-13

3.2.4 Maintainability___ 3-13

3.2.4.1 Corrective Maintenance___ 3-13

3.2.4.2 Preventive Maintenance___ 3-13

3.2.5 Environmental Conditions___ 3-13

3.2.5.1 Natural Environments____ 3-13

3.2.5.1.1 Ambient Air Temperature___ 3-13

3.2.5.1.2 Soil Temperature____ 3-13

3.2.5.1.3 Seismic Loads____ 3-14

3.2.5.1.4 Wind Loads___ 3-16

3.2.5.1.5 Snow Loads___ 3-16

3.2.5.1.6 Relative Humidity___ 3-16

3.2.5.1.7 Surface Precipitation___ 3-16

3.2.5.1.8 Hail Events___ 3-16

3.2.5.1.9 Sand and Dust___ 3-17

$\begin{array}{lll}3.2 .5 .1 .10 & \text { Solar Radiation____ 3.17 }\end{array}$

3.2.5.1.11 Glaze___ 3-17

3.2.5.1.12 Ashfall________-17

3.2.5.1.13 Dead Loads___ 3-17

3.2.5.1.14 Thermal Forces___ 3-17

3.2.5.1.15 Creep and Shrinkage Forces___ 3-17

3.2.5.1.16 Load Combinations and Allowable Stresses___ 3-17

3.2.5.2 Induced Environments___ 3-19

3.2.5.2.1 Waste Properties___ 3-19

3.2.5.2.2 Radiation Tolerance___ 3-19

3.2.5.2.2.1 Inside Pit Radiation Level___ 3-19

3.2.5.2.2.2 Background Pit Radiation Level___ 3-19

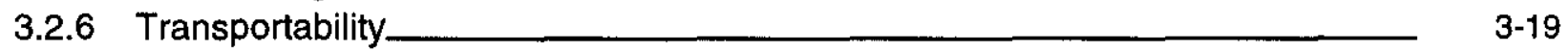

$\begin{array}{lll}3.2 .7 & \text { Flexibility and Expansion___ 3-20 }\end{array}$

\begin{tabular}{ll}
3.3 Design and Construction___ 3-20 \\
\hline
\end{tabular}

$\begin{array}{lll}\text { 3.3.1 Materials, Processes, and Parts___ 3-20 } & 3-20\end{array}$

3.3.1.1 Design and Construction Requirements____ 3-20

3.3.1.2 Cover Blocks Design and Construction Requirements___ 3-20

$\begin{array}{ll}\text { 3.3.1.3 Valve Position Sensor System__ 3-20 } & \text { 3.20 }\end{array}$

$\begin{array}{lll}\text { 3.3.1.4 Electrical Materials and Equipment_3-20 } & \text { 3.20 }\end{array}$

3.3.1.5 Field Control Equipment_ 3-20

3.3.1.6 Corrosion Resistance___ 3-20

3.3.1.7 Two-Way Full Ported Valves___ 3-20

$\begin{array}{lll}\text { 3.3.1.8 Three-Way Full Ported Valves____ 3-20 } & 3-20\end{array}$

$\begin{array}{lll}3.3 .1 .9 & \text { Optimization___ 3-20 }\end{array}$

$\begin{array}{lll}\text { 3.3.2 Electromagnetic Radiation___ 3-20 } & 3.20\end{array}$ 
3.3.2.1 Interference

3.3.2.2 Electromagnetic Force Interference___ 3-21

3.3.3 Identification and Marking___ 3-21

3.3.3.1 Labeling______-21

3.3.3.2 Master Equipment List___ 3-21

3.3.3.3 Operations and Maintenance Drawings____ 3-21

3.3.3.4 Valve Extension Handle Identification___ 3-21

3.3.4 Workmanship_____ 3-21

3.3.4.1 Valve Pit Piping Workmanship___ 3-21

3.3.4.2 Cover Blocks and New Pits Workmanship___ 3-21

3.3.5 Interchangeability___ 3-21

3.3.6 Safety__ 3-21

3.3.6.1 Nuclear Safety___ 3-21

3.3.6.1.1 Safety Classification___ 3-21

3.3.6.1.2 Flammable Gas Hazardous Environment___ 3-21

3.3.7 Human Performance/Human Engineering___ 3-22

3.3.7.1 Control Devices___ 3-22

3.3.7.2 Display Devices____ 3-22

3.3.7.3 Valve Actuator Handwheel___ 3-22

3.4 Documentation___ 3-22

3.4.1 Document Control___ 3-22

3.4.2 Drawings Preparation___ 3-22

3.5 Logistics___ 3-22

3.5.1 Maintenance___ 3-22

3.5.1.1 Fully Remote Maintenance and Operation___ 3-22

3.5.1.2 Limited Contact Maintenance and Operation___ 3-22

3.5.1.3 Full Contact Maintenance and Operation___ 3-22

3.5.1.4 Dome Loading Constraints____ 3-22

$\begin{array}{ll}\text { 3.5.1.5 Valve Maintenance___ 3-22 } & 3-23\end{array}$

3.5.2 Supply___ 3-23

3.5.2.1 Parts and Components____ 3-23

3.5.3 Facilities and Facility Equipment__ 3-23

$\begin{array}{lll}3.6 & \text { Personnel and Training___ 3-23 }\end{array}$

$\begin{array}{ll}\text { 3.7 Major Component Characteristics___ 3-23 } & 3-23\end{array}$

3.7.1 New Process Pits and Cover Blocks___ 3-23

3.7.1.1 Performance Characteristics___ 3-23

3.7.1.1.1 Confine Waste Leakage within the Transfer Associated Structures_____ 3-23

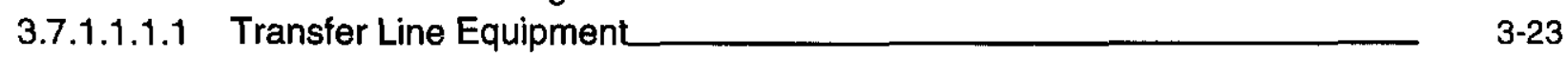

$\begin{array}{lll}\text { 3.7.1.1.1.2 Equipment in Process Pits___ 3-23 } & \text { 3.23 }\end{array}$

3.7.1.1.1.3 Process Pit Liner___ 3-23

$\begin{array}{lll}\text { 3.7.1.1.1.4 Release of Contamination___ 3-23 } & 3.23\end{array}$

3.7.1.1.1.5 Leak Detection Equipment Location____ 3-23

3.7.1.1.1.6 Low-Point Drain System____ 3-23

$\begin{array}{lll}\text { 3.7.1.1.1.7 Radiation Shielding___ 3-23 } & \text { 3.23 }\end{array}$

3.7.1.1.1.8 Cover Block Rain Intrusion___ 3-24

$\begin{array}{lll}\text { 3.7.1.1.1.9 Protective Coating___ 3-24 } & \end{array}$ 
3.7.1.1.1.10 Low-Point Drain System Capabilities___ 3-24

3.7.1.2 Physical Characteristics____ 3-24

3.7.1.2.1 Reinforced Concrete_____ 3-24

3.7.1.2.2 Process Pit Wall Nozzles___ 3-24

3.7.1.2.3 Process Pit Spare Nozzles___ 3-24

$\begin{array}{lll}\text { 3.7.1.2.4 Grounding Systems____ 3-24 } & 3.24\end{array}$

3.7.1.2.5 Cover Blocks Penetrations___ 3-24

3.7.1.2.6 Cover Blocks Rain Ingress___ 3-24

$\begin{array}{ll}\text { 3.7.1.2.7 Cover Blocks for Pump Pits__ 3-24 } & \text { 3.24 }\end{array}$

3.7.1.2.8 Gas Sampling Ports___ 3-25

3.7.1.2.9 Gas Sampling Ports Material____ 3-25

3.7.1.2.10 Gas Sampling Port Covers___ 3-25

$\begin{array}{lll}\text { 3.7.1.2.11 Lifting Mechanism___ 3.25 } & 3.25\end{array}$

3.7.1.2.12 Cover Blocks Alignment__ 3-25

3.7.2 Valve Position Sensor Systems___ 3-25

3.7.2.1 Performance Characteristics___ 3-25

3.7.2.1.1 Monitor Valve Position___ 3-25

3.7.2.1.1.1 Indicate Valve Limit Position___ 3-25

3.7.2.1.1.2 Active Valve Indication___ 3-25

3.7.2.1.1.3 Valve Position Transmission___ 3-25

3.7.2.1.1.4 Valve Position Indication Accuracy___ 3-25

3.7.2.2 Physical Characteristics____ 3-25

$\begin{array}{lll}\text { 3.7.2.2.1 Hardware Proximity___ 3-25 } & 3-25\end{array}$

3.7.2.2.2 Hardware Exposure to Environment__ 3-26

3.7.2.2.3 Hardware Removability___ 3-26

3.7.2.2.4 Hardware Capabilities____ 3-26

3.7.3 Pit Manifolds Sitework - New Pits___ 3-26

3.7.3.1 Performance Characteristics___ 3-26

3.7.3.1.1 Site Transfer Associated Structures___ 3-26

3.7.3.1.1.1 Site Soils____ 3-26

3.7.3.1.1.2 Access Control Fencing___ 3-26

3.7.3.1.1.3 Existing Lighting Extended___ 3-26

3.7.3.1.1.4 Lighting Control___ 3-26

3.7.3.2 Physical Characteristics____ 3-26

3.7.3.2.1 Disturbed Areas Stabilized___ 3-26

3.7.3.2.2 Pole-Mounted Luminaries___ 3.26

$\begin{array}{lll}\text { 3.7.3.2.3 Luminary Glare___ 3-26 } & 3.26\end{array}$

3.7.4 Pit Drain Seal Assemblies____ 3-26

3.7.4.1 Performance Characteristics___ 3-26

3.7.4.1.1 Position Pit Drain Seal Assembly___ 3-26

3.7.4.1.1.1 Twenty-four (24) Hour Holdup of Ten Gallons__ 3-26

$\begin{array}{lll}3.7 .4 .1 .1 .2 & \text { Vapor Seal_____ 3-27 }\end{array}$

3.7.4.1.1.3 Retractable Drain Seal___ 3-27

3.7.4.1.1.4 Drainage to the Tank_- 3-27

3.7.4.2 Physical Characteristics____ 3-27

$\begin{array}{lll}\text { 3.7.4.2.1 Operation Above Cover Block_ 3-27 } & \text { 3.27 }\end{array}$ 
3.7.4.2.2 Visible Indication of Position_ 3-27

3.7.4.2.3 Configuration to Prevent Drain Plugging__ 3-27

$\begin{array}{lll}\text { 3.7.4.2.4 Electrical Grounding Jumper__ 3-27 } & \text { 3.27 }\end{array}$

3.7.4.2.5 Configuration to Prevent Inadvertent Manipulation__ 3-27

4 SYSTEM QUALIFICATION PROVISIONS__ 4-1

4.1 General__ 4-1

4.1.1 Responsibility for Inspections__ 4-1

4.1.2 Special Tests and Examinations___ 4-1

4.2 Quality Conformance Inspections.__ 4-1

5 NOTES_ 5-1

5.1 Definitions_ 5-1

5.1.1 Manifold 5-1

5.1.2 Pit Nozzle_ 5-1

5.1.3 Equivalent Length__ 5-1

5.1.4 Glaze_ 5-1

5.2 Acronym List 5.1

5.3 Applicable Documents__ 5-2

\section{Appendices}

APPENDIX A FUNCTIONAL FLOW BLOCK DIAGRAMS (FFBDS)__ A-1

APPENDIX B REQUIREMENTS BASIS___ B-1 
HNF-SD-W314-PDS-002, Rev. 3

\section{List of Tables}

3-1 Manifold/Jumper Configuration Details__ 3-2

3-2 Chemical Composition Range____ 3-9

3-3 Radionuclide Concentrations____ 3-11

3-4 Response Spectra__ 3-15

3-5 Response Level_____ 3-16

4-1 Quality Conformance Inspection Matrix 4-3

B-1 Requirements Basis___ B-2 
HNF-SD-W314-PDS-002, Rev. 3

\section{SCOPE}

\subsection{Description}

This specification establishes the design basis for the performance, design development, and test requirements for upgrading six (6) valve pits, twenty-eight (28) pump pits, three (3) drain pits, two (2) feed pump pits, and providing two (2) new valve pits located in the 200 East Area The upgrades will be provided to support privatization waste transfers to the 241-AP Tank Farm and to add waste routing flexibility by deleting the need to reposition jumpers for various waste transters. The upgrades consist of new valve manifolds, valve position sensor systems, rupture disks, pressure transmitters and associated hardware, new floor drain seal assemblies, pit cover blocks, and new pits as required.

\subsection{Classification}

Not applicable to this specification. 


\section{APPLICABLE DOCUMENTS}

See Section 5.3 for notes regarding applicable documents.

\subsection{DOE Documents}

DOE 5480.20A, Personnel Selection, Qualification, Training, and Staffing Requirements at DOE Reactor and Non-Reactor Nuclear Facilities, 1/15/94.

DOE 6430.1A, General Design Criteria, United States Department of Energy, 4/6/89.

DOE-STD-1020, Natural Phenomena Hazards Design and Evaluation Criteria for Department of Energy Facilities, Jan. 1996.

\subsection{Code of Federal Regulations}

Not applicable to this specification.

\subsection{Tank Farm Contractor Documents}

HNF-IP-0842, RPP Administration, Rev. Oa.

HNF-PRO-242, Engineering Drawing Requirements, Rev. 1.

HS-BS-0084, Jumper Fabrication, Rev. B, Amendment 2, September 12, 1995.

RPP-PRO-224, Document Control Program Standards, Rev. 0.

RPP.PRO-233, Review and Approval of Documents, Rev. 0.

RPP-PRO-709, Preparation and Control Standards for Engineering Drawings, Rev. 0.

WHC-SD-WM-SARR-016, Tank Waste Compositions and Atmospheric Dispersion Coefficients for use in Safety Analysis Consequence Assessments, Rev. 2.

\subsection{Project W-314 Documents}

HNF-SD-W314-PDS-001, Project Development Specification for Transfer Piping, Rev. 3.

HNF-SD-W314-PDS-003, Project Development Specification for Pit Leak Detection, Rev. 3.

HNF-SD-W314-PDS-004, Project Development Specification for Master Pump Shutdown System, Rev. 3.

HNF-SD-W314-PICD-001, Project Interface Control Document for Project W-314, Rev. 2.

HNF-SD-W314-QAPP-001, Quality Assurance Program Plan for Project W-314.

\subsection{Codes and Standards}


ACl-301, Standard Specifications for Structural Concrete, American Concrete Institute.

ACl-318, Building Code Requirements for Reinforced Concrete, American Concrete Institute.

ACl-349, Code Requirements for Nuclear Safety Related Concrete Structures and Commentary, American Concrete Institute.

ANSI B16.34, Valves-Flanged, Threaded, and Welding End.

API 598, Valve Inspection and Testing.

ASCE 7-95, Minimum Design Loads for Buildings and Other Structures, American Society of Civil Engineers.

ASME B31.3, Process Piping.

FM, Approval Guide Building Materials, Factory Mutual System.

ICRP Publication 37, Cost Benefit Analysis in the Optimization of Radiological Protection.

MIL-STD-1472E, Human Engineering Design Criteria for Military Systems, Equipment and Facilities, U.S. Department of Defense.

NFPA 70, National Electrical Code, National Fire Protection Association.

NUREG 0700, Human System Interface Design Review Guideline.

UBC, Uniform Building Code.

UCRL-15910, Design and Evaluation Guidelines for DOE Facilities Subject to Natural Phenomena Hazards, University of California Research Laboratory.

UL 508A, Outline of Investigation for Industrial Control Panels, Underwriters Laboratories.

\subsection{Other Documents}

MSS-SP-61, Manufacturers Standardization Society, Standard Practice SP-61 for Pressure Testing of Steel Valves.

\subsection{Drawings}

H-2-32420, Assembly - Horizontal \& Vertical 2" Connector, Rev. 10.

H-2-32430, Assembly Horiz. \& Vert. Conn. 3" Connector, Rev. 9.

H-2-90185, Male Nozzle 2" PUREX, Rev. 4.

H-2-90186, Male Nozzle 3" PUREX, Rev. 4. 
HNF-SD-W314-PDS-002, Rev. 3

\section{REQUIREMENTS}

\subsection{Item Definition}

The valve manifold piping systems provide a confined routing for the transfer of tank waste between storage locations. The valve manifolds addressed by this specification are discrete segments of the Hanford tank farm waste transfer system.

This specification covers the design basis for the installation of manifolds/jumpers in the following pits:

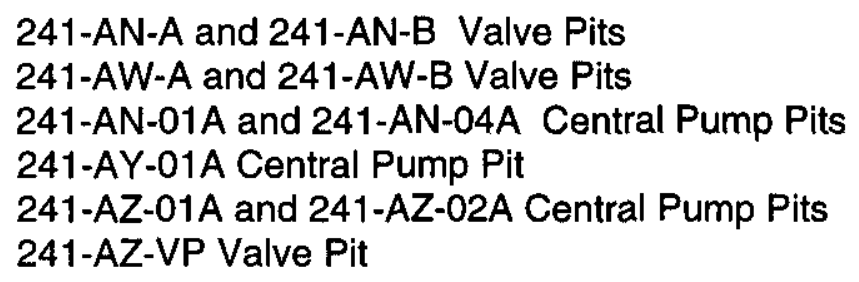

This specification also covers the design basis for the installation of valve position sensor systems in the following pits:

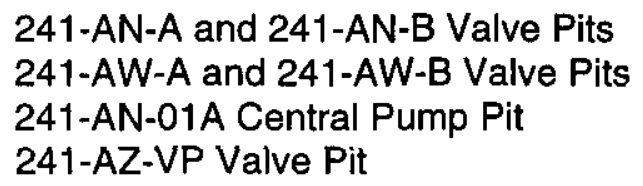

This specification also covers the design basis for the installation of new pit cover blocks in the following pits:

241-AN-A and 241-AN-B Valve Pits 241-AW-A and 241-AW-B Valve Pits 241-AZ-VP Valve Pit 214-AN-04D Slurry Receiver Pit

This specification also covers the design basis for the installation of new process pits (241-AZ-VP Valve Pit and 241-AN-04D Slurry Receiver Pit), as required.

This specification also covers the design basis for the installation of new floor drain seal assemblies in the following pits:

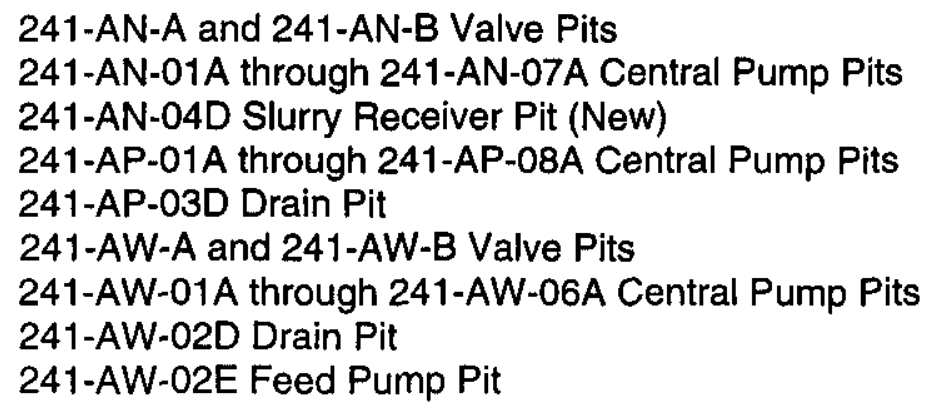


241-AY-01A and 241-AY-02A Central Pump Pits

241-AZ-01A and 241-AZ-02A Central Pump Pits

241-AZ-VP Valve Pit (New)

241-SY-01A through 241-SY-03A Central Pump Pits

241-SY-A and 241-SY-B Valve Pits

241-SY-02D Drain Pit

241-SY-02E Feed Pump Pit

Note: The existing cover blocks for 241-AZ-01A and 241-AZ-02A, 241-AY-01A and 241-AY-02A, 241-AN-01A through 241-AN-07A, 241-AP-01A through 241-AP-08A, 241-AW-01A through 241-AW-06A, and 241-SY-01A through 241-SY-03A Central Pump Pits; 241-SY-A and 241-SY-B Valve Pits; 241-AP-03D, 241-AW-02D, and 241-SY-02D Drain Pits; and 241-AP-02E and 241-SY-02E Feed Pump Pits will not be replaced, therefore the requirements in this specification associated with fabrication and installation of new cover blocks do not apply.

The above pits are designed to route tank waste between tanks within the tank farms in which they are located and also between specific tank farms and adjacent facilities via the waste transfer lines. Control over transfers is currently achieved through the configuration of the pit jumpers, i.e., which pit nozzles are tied together and the alignment of the valves on the jumpers, within the pits. Routing can be changed through the valves in the jumpers within the pits.

The nozzle configurations for the valve manifolds within the above pits are shown in the Table 3-1. Valve manifolds shall be connected only to the 3-inch transfer system, except as noted in this table. All applicable 3 -inch nozzles inside a pit will be connected by a manifold assembly while all applicable 2-inch nozzles will be connected by a separate manifold assembly. Isolation/process blanks identified in Table 3-1 are within Project W-314. If during construction, any unused nozzle is discovered to not have an isolation/process blank, one shall be provided and installed.

The valve manifolds, the pit cover blocks, and existing pits provide the primary and secondary confinement of the waste. The major components of the pit upgrades are as follows: (1) valve pit manifolds; (2) valve position sensor systems; (3) pit cover blocks, as required, (4) new process pit(s), as required, (5) pit floor drain seal assemblies, as required, and (6) tank farm lighting, as required. Note that pit cover blocks for 241-AZ-01A, 241-AZ-02A, 241-AY-01A, 241-AY-02A, 241-AN-01A, and 241-AN-04A Central Pump Pits and 241-AP Valve Pit will not be replaced, but modified as necessary for valving, leak detection, and access. The components of the pit upgrades and their relationship to the rest of the W-314 Waste Transfer System are shown graphically in Figure 3-1. The functional and physical relationship of the valve manifolds, valve position sensor system, and cover blocks to the tank farm waste transfer system is described in paragraphs 3.1.1 and 3.1.2.

Table 3-1 Manifold/Jumper Configuration Details

\begin{tabular}{|l|l|l|l|}
\hline Nozzle No. & Size & Pipe No. & Descriptions \\
\hline Location: $241-$ AN-A Valve Pit & \\
\hline L1 & $3^{\prime \prime}$ & 3" SN-267 & To Central Pump Pit 07A (A) \\
\hline L2 & $2^{\prime \prime}$ & & Isolation Blank \\
\hline L3 & $2^{\prime \prime}$ & 2" SL-167 $^{\prime \prime}$ & To Central Pump Pit 07A (B) \\
\hline
\end{tabular}


Table 3-1 Manifold/Jumper Configuration Details (Continued)

\begin{tabular}{|c|c|c|c|}
\hline Nozzle No. & Size & Pipe No. & Descriptions \\
\hline$L 4$ & $2^{\prime \prime}$ & & Isolation Blank \\
\hline L5 & $2^{\prime \prime}$ & 2" SL-164 & To Central Pump Pit 04A (B) \\
\hline$L 7$ & $2 "$ & 2" SL-165 & To Central Pump Pit 05A (B) \\
\hline L9 & $2^{\prime \prime}$ & 2" SL-166 & To Central Pump Pit 06A (B) \\
\hline L10 & $2^{\prime \prime}$ & & Isolation Blank \\
\hline L11 & $3^{\prime \prime}$ & & Isolation Blank \\
\hline$L 12$ & 3" & & Isolation Blank \\
\hline L13 & $3^{\prime \prime}$ & & Isolation Blank \\
\hline L14 & $3^{\prime \prime}$ & 3" SN-266 & To Central Pump Pit 06A (A) \\
\hline L15 & 3" & 3" SN-264 & To Central Pump Pit 04A (A) \\
\hline L16 & $3^{\prime \prime}$ & 3" SN-265 & To Central Pump Pit 05A (A) \\
\hline$L 17$ & $2^{\prime \prime}$ & & Process blank (existing) \\
\hline L18 & $2^{\prime \prime}$ & & Isolation Blank \\
\hline L19 & $3 "$ & 3" SN-268 & To Valve Pit AN-B (R19) \\
\hline L21 & $3^{\prime \prime}$ & New 3" Flush & Note: only new nozzle installed. \\
\hline \multicolumn{4}{|c|}{ Location: 241-AN-B Valve Pit } \\
\hline R1 & $3^{\prime \prime}$ & & Isolation Blank \\
\hline $\mathrm{R} 2$ & $2^{\prime \prime}$ & & Isolation Blank \\
\hline R3 & $2 "$ & & Isolation Blank \\
\hline R4 & $2^{\prime \prime}$ & & Isolation Blank \\
\hline R5 & $2^{\prime \prime}$ & 2" SL-161 & To Central Pump Pit 01A (B) \\
\hline R7 & $2 "$ & 2" SL-162 & To Central Pump Pit 02A (B) \\
\hline R9 & $2 "$ & 2" SL-163 & To Central Pump Pit 03A (B) \\
\hline R10 & $2 "$ & & Isolation Blank \\
\hline$R 11$ & 3" & & Isolation Blank \\
\hline $\mathrm{R} 12$ & $3^{\prime \prime}$ & & Isolation Blank \\
\hline R13 & $2 "$ & & Isolation Blank \\
\hline R14 & $3 "$ & 3" SN-263 & To Central Pump Pit 03A (A) \\
\hline R15 & 3" & 3" SN-261 & To Central Pump Pit 01A (A) \\
\hline$R 16$ & $3^{\prime \prime}$ & 3" SN-262 & To Central Pump Pit 02A (A) \\
\hline R17 & $2 "$ & & Isolation Blank \\
\hline
\end{tabular}


Table 3-1 Manifold/Jumper Configuration Details (Continued)

\begin{tabular}{|c|c|c|c|}
\hline Nozzle No. & Size & Pipe No. & Descriptions \\
\hline R18 & $2^{\prime \prime}$ & & Isolation Blank \\
\hline R19 & $3^{\prime \prime}$ & $3^{\prime \prime}$ SN-268 & To Valve Pit AN-A (L19) \\
\hline F20 & $2^{\prime \prime}$ & New 2" Flush & From Blending Tank TK-301 \\
\hline \multicolumn{4}{|c|}{ Location: 241-AY-01A Central Pump Pit } \\
\hline U3 & $4^{\prime \prime}$ & Spare & Isolation Blank \\
\hline U8 & $2^{\prime \prime}$ & Spare & Isolation Blank \\
\hline U9 & $3^{\prime \prime}$ & Spare & Isolation Blank \\
\hline U12 & $2^{\prime \prime}$ & SL-505 & To Distributor (Existing) \\
\hline U13 & $3^{\prime \prime}$ & New SN-635 & Process blank \\
\hline U13A & $2^{\prime \prime}$ & New $2 "$ DR & New SN-635 Encasement DR \\
\hline \multicolumn{4}{|c|}{ Location: 241-AY-02A Central Pump Pit } \\
\hline U5 & $3^{\prime \prime}$ & New SN-633 & Process blank \\
\hline U12 & $3^{\prime \prime}$ & New SN-635 & Process blank \\
\hline \multicolumn{4}{|c|}{ Location: 241-AZ-01A Central Pump Pit } \\
\hline U11 & $3^{\prime \prime}$ & New SN-631 & Process blank \\
\hline U11A & $2^{\prime \prime}$ & New 2" Drain & New SN-631 Encasement Drain \\
\hline U12 & $3^{\prime \prime}$ & New SN-632 & Process blank \\
\hline U12A & $2 "$ & New 2" DR & New SN-632 Encasement DR \\
\hline \multicolumn{4}{|c|}{ Location 241-AZ-02A Central Pump Pit } \\
\hline U13 & 3" & New SN-631 & Process blank \\
\hline \multicolumn{4}{|c|}{ Location: 241-AW-A Valve Pit } \\
\hline L1 & 3" & 3" SN-267 & To Pump Pit 241-AW-02A (J) \\
\hline $\mathrm{L} 2$ & 3" & 3" SN-220 & From 204-AR \\
\hline L3 & $2 "$ & 2" SL-168 & From Evap. Bldg. 242-A (18) \\
\hline L4 & $2^{\prime \prime}$ & 2" SL-510 & To Valve Pit 241-AP (2) \\
\hline L5 & $2 "$ & TBD & $\begin{array}{l}\text { Failed line to be replaced by Project W-521. New } \\
\text { nozzle for Project W-521. }\end{array}$ \\
\hline L7 & $2^{\prime \prime}$ & 2" SL-163 & To Pump Pit 241-AW-03A (B) \\
\hline L9 & $2 "$ & 2" SL-165 & To Pump Pit 241-AW-05A (B) \\
\hline L10 & $2 "$ & Spare & Vapor Seal \\
\hline L11 & 3" & 3" Flush & New for Project W-521 \\
\hline
\end{tabular}


HNF-SD-W314-PDS-002, Rev. 3

Table 3-1 Manifold/Jumper Configuration Details (Continued)

\begin{tabular}{|c|c|c|c|}
\hline Nozzle No. & Size & Pipe No. & Descriptions \\
\hline L12 & 3" & 3" P-V021 & Isolation blank \\
\hline L13 & $3^{\prime \prime}$ & Spare & Vapor Seal \\
\hline L14 & $3^{\prime \prime}$ & 3" SN-263 & To Pump Pit 241-AW-03A (A) \\
\hline L15 & 3" & 3" SN-265 & To Pump Pit 241-AW-05A (A) \\
\hline L16 & 3" & 3" SN-261 & To Pump Pit 241-AW-01A (A) \\
\hline L17 & $11 / 2^{\prime \prime}$ & $11 / 2^{\prime \prime}$ Flush & Process blank \\
\hline L18 & $2^{\prime \prime}$ & 2" SL-169 & Vapor Seal \\
\hline L19 & 3" & 3" SN-271 & Vapor Seal \\
\hline \multicolumn{4}{|c|}{ Location: 241-AW-B Valve Pit } \\
\hline R1 & 3" & 3" SN-268 & To Pump Pit 241-AW-02A (H) \\
\hline $\mathrm{R} 2$ & 3" & 3" SN-167 & Process blank \\
\hline R3 & $2^{\prime \prime}$ & 2" SL-167 & To Evap. Bldg. 242-A (19) \\
\hline R4 & $2^{\prime \prime}$ & 2" SL-509 & To Valve Pit 241-AP (1) \\
\hline R5 & $2^{\prime \prime}$ & 2" SL-161 & Process blank \\
\hline R7 & $2^{\prime \prime}$ & 2" SL-164 & To Pump Pit 241-AW-04A (B) \\
\hline R9 & $2^{\prime \prime}$ & 2" SL-166 & To Pump Pit 241-AW-06A (B) \\
\hline$R 11$ & $3^{\prime \prime}$ & 3" P-V023 & Process blank \\
\hline R12 & 3" & 3" P-V022 & Process blank \\
\hline R13 & $3^{\prime \prime}$ & Spare & Vapor Seal \\
\hline $\mathrm{R} 14$ & $3^{\prime \prime}$ & 3" SN-264 & To Pump Pit 241-AW-04A (A) \\
\hline R15 & 3" & 3" SN-266 & To Pump Pit 241-AW-06A (A) \\
\hline R16 & 3" & 3" SN-262 & Process blank \\
\hline $\mathrm{R} 17$ & $11 / 2$ & $11 / 2^{\prime \prime}$ Flush & Process blank \\
\hline R18 & $2 "$ & 2" SL-169 & Vapor Seal \\
\hline R19 & 3" & 3" SN-271 & Vapor Seal \\
\hline R21 & $3^{\prime \prime}$ & 3" Flush Line & New nozzle for Project $W-521$ \\
\hline \multicolumn{4}{|c|}{ Location: 241-AN-01A Central Pump Pit } \\
\hline$A$ & $3^{\prime \prime}$ & SN-261 & To Valve Pit 241-AN-B (R15) \\
\hline $\mathrm{H}$ & $3^{\prime \prime}$ & SNL-3150 & From cross-site tie-in (new) \\
\hline $\mathrm{C}$ & $2^{\prime \prime}$ & 2" DR & New SNL-3150 Drain \\
\hline D & $3^{\prime \prime}$ & SN-630 & From 241-AZ-VP Valve Pit (A) (new) \\
\hline
\end{tabular}


Table 3-1 Manifold/Jumper Configuration Details (Continued)

\begin{tabular}{|c|c|c|c|}
\hline Nozzle No. & Size & Pipe No. & Descriptions \\
\hline $\mathrm{J}$ & 2" & 2" DR & New SN-630 Drain \\
\hline E & $2^{\prime \prime}$ & & Process blank \\
\hline$F$ & $2^{\prime \prime}$ & & Process blank \\
\hline $3 A$ & 3" & & To Transfer Pump \\
\hline \multicolumn{4}{|c|}{ Location: 241-AN-04A Central Pump Pit } \\
\hline C & $2 "$ & & Isolation blank \\
\hline D & $3^{\prime \prime}$ & SN-636 & Isolation blank \\
\hline $\mathrm{H}$ & 2" & SN-636 ENC DR & New SN-636 encasement drain \\
\hline E & 2" & & Process blank \\
\hline$F$ & 2" & & Process blank \\
\hline \multicolumn{4}{|c|}{ 241-AZ-VP Valve Pit } \\
\hline$A$ & 3" & SN-630 & To Pump Pit 241-AN-01A (D) \\
\hline$B$ & 2" & SN-637 ENC DR & New Encasement Drain \\
\hline C & 3" & SN-637 & To New AP-A Valve Pit (Project W-521) \\
\hline$D$ & $2 "$ & SN-634 ENC DR & New Encasement Drain \\
\hline $\mathrm{E}$ & 3" & SN-634 & To New AP-A Valve Pit (Project W-521) \\
\hline$F$ & 3" & $\mathrm{SN}-633$ & To Pump Pit 241-AY-02A (U5) \\
\hline G & 2" & SN-633 ENC DR & New Encasement Drain \\
\hline $\mathrm{H}$ & 3" & $\mathrm{SN}-632$ & To Pump Pit 241-AZ-01A (U12) \\
\hline
\end{tabular}

Note: In the description, block numbers and/or letters in parenthesis $(x)$ indicate the nozzle location in the referenced pit. 
Figure 3-1. Relationship of Pit Upgrades to W-314 Transfer Waste System

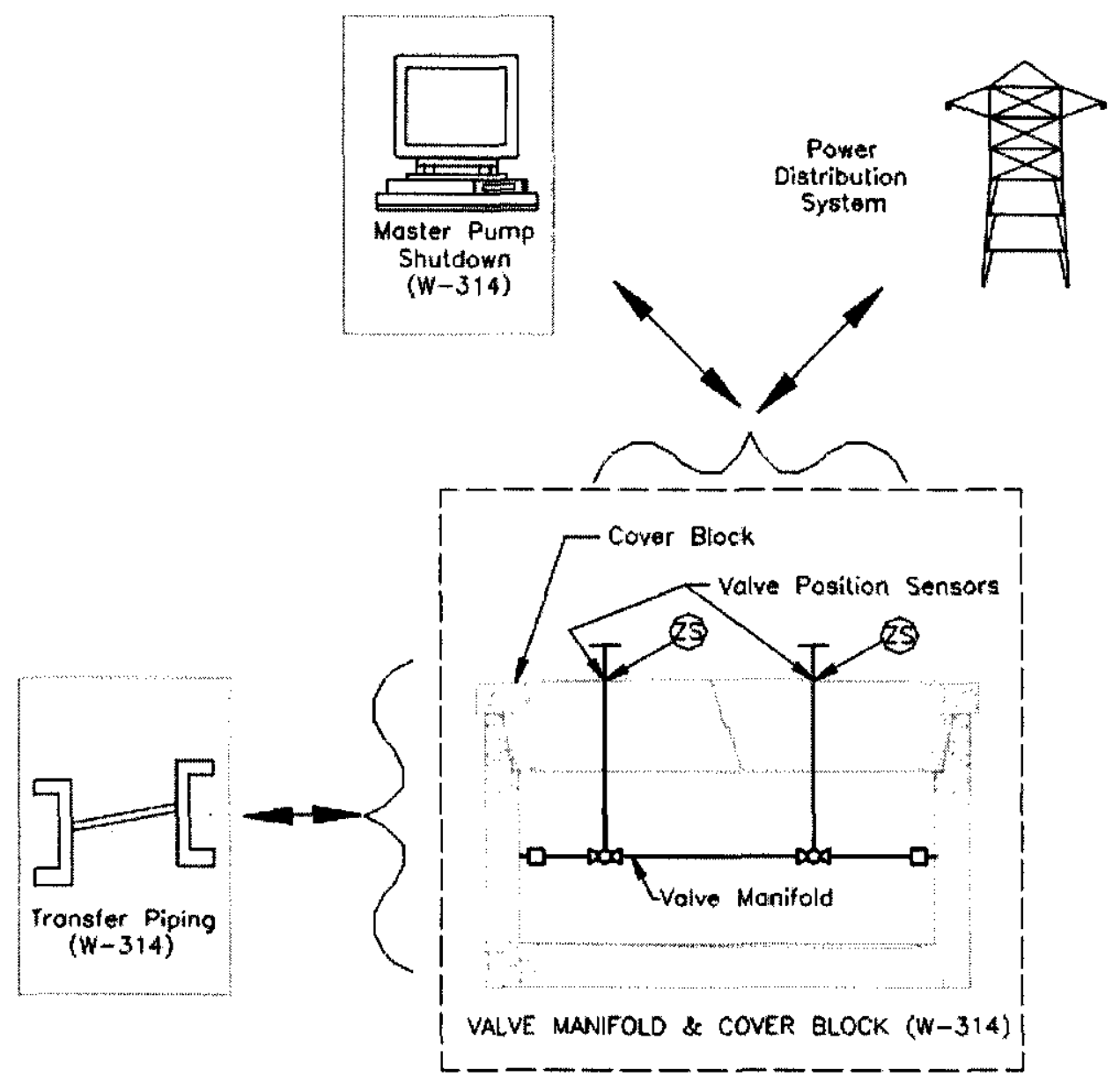

\subsubsection{Item Diagrams}

The functions of the valve pit manifolds were developed during the functional analysis of the W-314 Waste Transfer System. The valve pit manifolds and their major components must meet the requirements of the following functions:

- Function 3.1.1

- Function 3.1.2

- Function 3.1.3

. Function 3.1.4

- Function 3.1.8

- Function 3.2.1

- Function 3.2.6
Confine Waste Within Valve Manifolds and Jumpers Position Manifold Valves

Confine Flush Within Valve Manifolds and Jumpers Monitor Valve Position Position Pit Drain Seal Assembly Confine Waste Leakage Within Transfer-Associated Structures Site Transfer Associated Structures

Appendix A contains the Functional Flow Block Diagrams (FFBD) that include diagrammatic presentations of the above functions and their inter-relationship with other applicable functions of the transfer waste system. 
HNF-SD-W314-PDS-002, Rev. 3

Appendix B contains the Requirements Allocation Sheets (RASs) for all requirements in this specification.

\subsubsection{Interface Definition}

Functional and physical interfaces for the valve pit manifolds are described in the Project W-314 Project interface Control Document (PICD), HNF-SD-W314-PICD-001.

In addition, the design shall be coordinated with the systems identified in the following Project Development Specifications (PDS):

- HNF-SD-W314-PDS-001 PDS for Transfer Piping

- HNF-SD-W314-PDS-003 PDS for Pit Leak Detection

. HNF-SD-W314-PDS-004 PDS for MPS System

\subsubsection{Major Component List}

In addition to new valve pit manifolds, the upgrades to the valve and pump pits have the following major components:

- Valve position sensor systems.

- Pit cover blocks.

- New process pit(s)

- Site work

- Pit floor drain seal assemblies

\subsection{Characteristics}

\subsubsection{Performance Characteristics}

The valve manifold piping system and the piping in 214-AN-04D Slurry Receiver Pit and components shall meet the performance requirements stated in this section.

3.2.1.1 Confine Waste Within Valve Manifolds and Jumpers. (FFBD Function 3.1.1) After the transfer pump has started, the piping system shall confine the waste during transfers.

3.2.1.1.1 Corrosion/Erosion. The piping system components shall support a corrosion/ erosion allowance of $0.01 \mathrm{~mm}$ per year $(0.4 \mathrm{mil}$ per year) for the life of the system.

3.2.1.1.2 Reynolds Number. The piping system components shall be capable of transferring the waste at a Reynolds number greater than or equal to 20,000 .

3.2.1.1.3 Head Loss. The valve manifold/jumper piping system shall have a head loss no greater than 10 meters per 100 meters (10 feet per 100 feet) of equivalent length of 3 in Sch 40 pipe.

3.2.1.1.4 Design Pressure. The valve manifold/jumper piping system components shall be capable of supporting a design pressure of 400 psig. For interconnecting piping that have design pressures exceeding $400 \mathrm{psig}$, it is the owner/operator responsibility to verify that the 
most severe condition of coincidental internal pressure and temperature expected during service does not exceed the design conditions of the valve manifold.

3.2.1.1.5 WT-SLL-3160 Design Pressure. The connection of WT-SLL-3160 to Riser 10 on tank 241-AN-104 shall be capable of supporting a design pressure of $10.27 \mathrm{Mpa}$ (1490 psi) up to and including the last point of isolation prior to entry into the riser.

3.2.1.1.6 WT-SLL-3160 Encasement Design Pressure. The WT-SLL-3160 line encasement shall have a design pressure of $50 \mathrm{psig}$ and a design temperature of $180^{\circ} \mathrm{F}$.

3.2.1.1.7 Temperature Maintenance. The valve manifold/jumper piping system components, as part of the waste transfer system, shall be capable of maintaining the temperature of the contents such that the temperature drop through the piping in the pit does not exceed $2^{\circ} \mathrm{C}(3$ $\left.{ }^{\circ} \mathrm{F}\right)$ during a waste transfer.

3.2.1.1.8 Compatibility with Waste. The piping system components shall be capable of withstanding the liquid waste chemical composition ranges listed in Table 3-2 and the waste radionuclide concentrations listed under the W-314 column in Table 3-3.

Table 3-2 Chemical Composition Range

\begin{tabular}{|c|c|c|c|c|}
\hline \multicolumn{5}{|c|}{ Retrieved waste } \\
\hline \multirow[t]{3}{*}{ Species } & \multicolumn{2}{|c|}{ DST } & \multicolumn{2}{|c|}{ SST } \\
\hline & \multicolumn{2}{|c|}{ Anion/cation } & \multicolumn{2}{|c|}{ Anion/cation } \\
\hline & $\min \mathrm{mol} / \mathrm{L}$ & $\max \mathrm{mol} / \mathrm{L}$ & $\min \mathrm{mol} / \mathrm{L}$ & $\max \mathrm{mol} / \mathrm{L}$ \\
\hline $\mathrm{Ag}$ & 0 & 0.0013 & - & - \\
\hline $\mathrm{Al}$ & 0.05 & 1.1 & 0.029 & 0.5 \\
\hline As & 0 & 0.0066 & - & - \\
\hline B & 0 & 0.013 & - & - \\
\hline $\mathrm{Ba}$ & 0 & 0.0004 & 0 & 0.0014 \\
\hline $\mathrm{Bi}$ & - & - & 0 & 0.076 \\
\hline $\mathrm{Ca}$ & 0.0014 & 0.1 & 0 & 0.17 \\
\hline $\mathrm{Cd}$ & 0 & 0.0074 & 0 & 0.0007 \\
\hline $\mathrm{Cr}$ & 0.0067 & 0.28 & 0.0001 & 0.091 \\
\hline $\mathrm{Cu}$ & 0 & 0.02 & - & - \\
\hline $\mathrm{Fe}$ & 0.0004 & 0.26 & 0.0057 & 0.89 \\
\hline $\mathrm{Hg}$ & 0 & $2.8 \mathrm{E}-05$ & 0 & 0.0001 \\
\hline $\mathrm{K}$ & 0.044 & 0.55 & 0.0002 & 0.0095 \\
\hline La, Nd & 0 & 0.0066 & 0 & 0.001 \\
\hline $\mathrm{Mg}$ & 0.0004 & 0.046 & - & - \\
\hline $\mathrm{Mn}$ & 0.0003 & 0.16 & 0.0009 & 0.41 \\
\hline
\end{tabular}


HNF-SD-W314-PDS-002, Rev. 3

Table 3-2 Chemical Composition Range (Continued)

\begin{tabular}{|c|c|c|c|c|}
\hline \multicolumn{5}{|c|}{ Retrieved waste } \\
\hline \multirow[t]{3}{*}{ Species } & \multicolumn{2}{|c|}{ DST } & \multicolumn{2}{|c|}{ SST } \\
\hline & \multicolumn{2}{|c|}{ Anion/cation } & \multicolumn{2}{|c|}{ Anion/cation } \\
\hline & $\min \mathrm{mol} / \mathrm{L}$ & $\mathrm{max} \mathrm{mol} / \mathrm{L}$ & $\min \mathrm{mol} / \mathrm{L}$ & $\max \mathrm{mol} / \mathrm{L}$ \\
\hline Mo & 0 & 0.0029 & - & - \\
\hline $\mathrm{Na}$ & 1.6 & 10.7 & 1.6 & 7.1 \\
\hline $\mathrm{Ni}$ & 0.0002 & 0.008 & 0 & 0.042 \\
\hline $\mathrm{Pb}$ & 0 & 0.004 & 0 & 0.12 \\
\hline $\mathrm{Pd}, \mathrm{Rh}$ & 0 & 0.0063 & - & - \\
\hline $\mathrm{Si}(\mathrm{SiO} 2)$ & 0.0024 & 0.028 & 0.0004 & 0.46 \\
\hline $\mathrm{Ti}$ & 0 & 0.002 & - & - \\
\hline$U$ & 0 & 0.0092 & - & - \\
\hline $\mathrm{Zr}(\mathrm{ZrO} 2)$ & 0 & 0.3 & 0 & 0.065 \\
\hline Acetate & - & - & 0 & 0.0055 \\
\hline Citrate & 0 & 0.03 & 0.0042 & 0.06 \\
\hline EDTA & 0 & 0.016 & 0 & 0.011 \\
\hline HEDTA & 0 & 0.021 & - & - \\
\hline $\mathrm{Fe}(\mathrm{CN}) 6$ & - & - & 0 & 0.025 \\
\hline $\mathrm{Cl}$ & 0.003 & 0.17 & 0 & 0.022 \\
\hline $\mathrm{CO} 3$ & 0.03 & 0.69 & 0.014 & 0.38 \\
\hline$F$ & 0.014 & 1 & 0.001 & 0.71 \\
\hline Fission product & 0 & 0.0001 & - & - \\
\hline NO2 & 0.1 & 1.8 & 0.0086 & 0.83 \\
\hline NOX(NO3) & 0.15 & 3.6 & 0.64 & 5.1 \\
\hline $\mathrm{OH}$ & 0.24 & 4.4 & 0.25 & 6.9 \\
\hline PO4 & 0 & 0.4 & 0.0007 & 3.8 \\
\hline SO4 & 0.003 & 0.16 & 0.01 & 0.22 \\
\hline TOC & 0 & 2 & - & - \\
\hline
\end{tabular}

DST = Double-shell tank

EDTA = Ethylenediametraacetic acid

HEDTA =n-(hydroxyethyl) -Ethylenediametraacetic acid

SST = Single-shell tank

TOC = Total organic carbon 
Table 3-3 Radionuclide Concentrations

\begin{tabular}{|c|c|c|c|}
\hline \multirow[t]{2}{*}{ Nuclide } & \multicolumn{3}{|c|}{ Nuclide Concentrations $(\mathrm{Bq} / \mathrm{L})$} \\
\hline & (a)All liquids & (a)All solids & (b)W-314 \\
\hline $14 \mathrm{C}$ & $2.3 E+05$ & $1.6 \mathrm{E}+05$ & $2.3 E+05$ \\
\hline $60 \mathrm{Co}$ & $9.5 \mathrm{E}+06$ & $4.9 E+08$ & 1.7E+08 \\
\hline $79 \mathrm{Se}$ & (c) & $1.7 E+04$ & $1.7 E+04$ \\
\hline $90 \mathrm{Sr}$ & $1.1 E+10$ & $2.9 E+12$ & $9.6 \mathrm{E}+11$ \\
\hline $90 Y$ & $1.1 E+10$ & $2.9 E+12$ & $9.6 E+11$ \\
\hline 99Tc & $1.7 E+07$ & $1.2 \mathrm{E}+10$ & $4.0 E+09$ \\
\hline $106 \mathrm{Ru}$ & $9.9 E+02$ & $7.2 E+04$ & $2.4 \mathrm{E}+04$ \\
\hline $125 \mathrm{Sb}$ & $3.4 E+04$ & $1.8 \mathrm{E}+08$ & $5.9 E+07$ \\
\hline 1291 & $2.0 E+04$ & $6.4 E+06$ & $2.1 E+06$ \\
\hline $134 \mathrm{Cs}$ & $6.1 \mathrm{E}+06$ & $9.4 E+06$ & $7.2 E+06$ \\
\hline $137 \mathrm{Cs}$ & $8.8 E+10$ & $1.0 \mathrm{E}+11$ & $9.2 E+10$ \\
\hline $144 \mathrm{Ce}$ & $9.1 E+00$ & $3.4 E+02$ & $1.2 E+02$ \\
\hline $147 \mathrm{Pm}$ & $3.6 \mathrm{E}+07$ & (c) & $3.6 \mathrm{E}+07$ \\
\hline 154Eu & $2.4 E+09$ & $1.1 E+10$ & $5.2 \mathrm{E}+09$ \\
\hline $155 \mathrm{Eu}$ & $5.9 E+07$ & $5.0 \mathrm{E}+06$ & $5.9 E+07$ \\
\hline $237 \mathrm{~Np}$ & $2.3 E+05$ & $9.9 E+08$ & $3.3 E+08$ \\
\hline $238 \mathrm{Pu}$ & $1.8 \mathrm{E}+06$ & $1.9 E+08$ & $6.4 \mathrm{E}+07$ \\
\hline $239 \mathrm{Pu}(\mathrm{d})$ & $3.6 \mathrm{E}+07$ & $1.6 \mathrm{E}+09$ & $5.5 E+08$ \\
\hline $241 \mathrm{Pu}$ & $2.6 \mathrm{E}+08$ & $3.8 \mathrm{E}+09$ & $1.4 E+09$ \\
\hline $241 \mathrm{Am}$ & $4.2 \mathrm{E}+07$ & $1.1 \mathrm{E}+10$ & $3.7 E+09$ \\
\hline $242 \mathrm{Cm}$ & $1.1 E+01$ & $2.0 \mathrm{E}+02$ & $7.3 E+01$ \\
\hline $244 \mathrm{Cm}$ & $4.2 E+05$ & $6.1 E+07$ & $2.0 \mathrm{E}+07$ \\
\hline
\end{tabular}

(a) From Table 1a., Van Keuren, J. C., 1996, Tank Waste Compositions and Atmospheric Dispersion Coefficients for Use in Safety Analysis Consequence Assessments, WHC-SD-WM-SARR-016, Rev. 2, Westinghouse Hanford Company, Richland, Washington.

(b) W-314 values represent a bounding mixture for design of $67 \%$ liquid and $33 \%$ solid, except for $14 \mathrm{C}$ and $155 \mathrm{Eu}$ where the maximum liquid value was used as it is higher than the mix and for $79 \mathrm{Se}$ and $147 \mathrm{Pm}$ where data is not available.

(c) No available data.

(d) The 239Pu activity concentration also includes $240 \mathrm{Pu}$. 
3.2.1.2 Position Manifold Valves. (FFBD Function 3.1.2) After the appropriate valve manifolds have been selected, the waste transfer system shall position the valves on the selected manifold.

3.2.1.2.1 Remote Valve Operation. The valve manifold/jumper valves shall be remote manually operated from above the pit cover blocks.

3.2.1.3 Confine Flush in Valve Manifolds and Jumpers. (FFBD Function 3.1.3) During the flush of the waste transfer system, the piping system shall confine the flush water.

3.2.1.3.1 Compatibility with Flush Water. The piping system components shall be capable of confining flush water with the following properties:

. Volumetric flow rate of $151-530 \mathrm{~L} / \mathrm{min}(40-140 \mathrm{gpm})$

. Temperature of $12.8-66^{\circ} \mathrm{C}\left(55-150^{\circ} \mathrm{F}\right)$

- $\mathrm{pH}$ range of no less than 7 and a maximum of 14 .

\subsubsection{Physical Characteristics}

3.2.2.1 Valve Piping System Features. The valve piping system shall have the following features:

a. Manual valve actuation from above the cover blocks (except for the encasement rupture disk isolation valve in 241-AN-04D Slurry Receiver Pit).

b. Valves shall be capable of being locked in position (except the WT-SLL-3160 block valve at Riser 10).

3.2.2.2 Valve Extension Handles. The pit valves shall be manually operated with valve operating extension rods extending through the pit cover blocks (except for the encasement rupture disk isolation valve in 241-AN-04D Slurry Receiver Pit).

3.2.2.3 PUREX Jumper Connector. The PUREX jumper connector assembly design will be in accordance with drawings $\mathrm{H}-2-32430$ and $\mathrm{H}-2-32420$. The pit nozzle shall be PUREX type design (reference drawings $\mathrm{H}-2-90185$ and $\mathrm{H}-2-90186$ ).

3.2.2.4 Jumper Manifold. Each jumper manifold shall have a valved nozzle to facilitate interface with the existing transfer piping system via flexible jumpers (Valve Pit 241-AP and the new 241-AZ-VP Valve Pit are excluded from this requirement).

3.2.2.5 Valve Position Indication System. The valve position indication system shall provide sufficient accuracy with respect to the actual valve position such that the selected flow path configuration is leak tight.

\subsubsection{Not used}

3.2.2.7 Valve Position Indicators. Indication of the valves position shall be visible when extension handles are removed.

3.2.2.8 Valve Lock Capacity. The valve operators shall be capable of being locked in place by a padlock that has a bail diameter of $5 / 16$ " and a minimum closed length of 1.5 inches.

\subsubsection{Reliability}


3.2.3.1 Design Life of Valve Manifold. The design life of the valve manifold/jumper piping system components shall be 12 years. The design life of the WT-SLL-3160 piping system components shall be 40 years.

3.2.3.2 Design Life of Cover Blocks. The design life of the cover blocks and the new 241-AZ-VP shall be 35 years.

3.2.3.3 Design Life of Slurry Receiver Pit. The design life of the Slurry Receiver Pit, 241-AN-04D, shall be 40 years.

3.2.3.4 Degree of Reliability. Valves shall be designed to operate properly following stagnant periods of up to one year.

3.2.3.5 Valve Life. Valves shall be capable of being operated up to 1000 cycles over its design life.

3.2.3.6 Fault Detection/lsolation. Designs shall provide for the detection and isolation of faults to systems, structures, and components as necessary in order to minimize the risks associated with faulty operation to plant, personnel and environment. Protection systems and associated instrumentation and controls shall be designed in accordance with DOE 6430.1A, Section 1660-99.0.2.

\subsubsection{Maintainability}

3.2.4.1 Corrective Maintenance. The repair or replacement of a failed components on the valve manifold/jumper shall be performed within 25 days.

3.2.4.2 Preventive Maintenance. The design of the valve manifold/jumper system shall ensure no preventive maintenance requiring pit entry.

\subsubsection{Environmental Conditions}

The systems and components covered by this specification shall be compatible with the environmental conditions listed below, as applicable. Performance Category (PC) 3 is assigned to safety class systems and PC1 is assigned to general service systems. The design and analysis of loads associated with existing systems shall, as a minimum, be performed to the design requirements of the existing system.

\subsubsection{Natural Environments}

3.2.5.1.1 Ambient Air Temperature. The ambient air (outside the pit) temperature range is $48.9^{\circ} \mathrm{C}\left(120^{\circ} \mathrm{F}\right)$ to $-35.5^{\circ} \mathrm{C}\left(-32^{\circ} \mathrm{F}\right)$, and with a maximum 24 hour differential of $28.9^{\circ} \mathrm{C}\left(52^{\circ} \mathrm{F}\right)$.

3.2.5.1.2 Soil Temperature. The minimum soil temperatures below ground surface is:

$$
\begin{array}{ll}
1.3 \mathrm{~cm}(0.5 \mathrm{in}): & -19.5^{\circ} \mathrm{C}\left(-3.0^{\circ} \mathrm{F}\right) \\
38 \mathrm{~cm}(15 \mathrm{in}): & -8.8^{\circ} \mathrm{C}\left(16.1^{\circ} \mathrm{F}\right) \\
92 \mathrm{~cm}(36 \mathrm{in}): & 0.8^{\circ} \mathrm{C}\left(33.5^{\circ} \mathrm{F}\right)
\end{array}
$$




\subsection{Seismic Loads. PC1 structures, systems, and components}

Earthquake load design of PC1 SSCs shall comply with the UBC, Seismic Zone 2B, for standard occupancy facilities.

\section{PC3 structures, systems, and components}

Earthquake load design of PC3 SSCs shall comply with DOE-STD-1020 by using dynamic analysis and site-specific design response spectra listed in Table 3-4.

New process pits and new cover blocks shall be designed, analyzed, procured, and constructed to $P C 3$ requirements.

Calculate elastic seismic response, Ds, by dynamic analysis using Response Level 2 damping values from Table $3-5$. 
HNF-SD-W314-PDS-002, Rev. 3

Table 3-4 Response Spectra

\begin{tabular}{|c|c|c|c|c|c|c|}
\hline \multicolumn{7}{|c|}{ Horizontal Response Spectra "g" } \\
\hline $\begin{array}{c}\text { Frequency } \\
\text { (Hertz) }\end{array}$ & \multicolumn{7}{|c|}{ Damping } \\
\cline { 2 - 7 } & $0.50 \%$ & $2 \%$ & $5 \%$ & $7 \%$ & $10 \%$ & $12 \%$ \\
\hline 100 & 0.26 & 0.26 & 0.26 & 0.26 & 0.26 & 0.26 \\
\hline 33.3 & 0.26 & 0.26 & 0.26 & 0.26 & 0.26 & 0.26 \\
\hline 13.3 & 0.57 & 0.48 & 0.41 & 0.38 & 0.36 & 0.35 \\
\hline 10 & 0.77 & 0.59 & 0.47 & 0.43 & 0.38 & 0.36 \\
\hline 5 & 1.04 & 0.76 & 0.58 & 0.52 & 0.45 & 0.42 \\
\hline 3.3 & 0.98 & 0.72 & 0.54 & 0.48 & 0.42 & 0.39 \\
\hline 2 & 0.74 & 0.55 & 0.41 & 0.37 & 0.33 & 0.30 \\
\hline 1 & 0.45 & 0.34 & 0.26 & 0.23 & 0.21 & 0.19 \\
\hline 0.5 & 0.22 & 0.17 & 0.13 & 0.12 & 0.11 & 0.10 \\
\hline 0.25 & 0.08 & 0.06 & 0.05 & 0.05 & 0.04 & 0.04 \\
\hline
\end{tabular}

\begin{tabular}{|c|c|c|c|c|c|c|}
\hline \multicolumn{7}{|c|}{ Vertical Response Spectra "g" } \\
\hline \multirow{2}{*}{$\begin{array}{c}\text { Frequency } \\
\text { (Hertz) }\end{array}$} & \multicolumn{7}{|c|}{ Damping } \\
\cline { 2 - 7 } & $0.50 \%$ & $2 \%$ & $5 \%$ & $7 \%$ & $10 \%$ & $12 \%$ \\
\hline 100 & 0.18 & 0.18 & 0.18 & 0.18 & 0.18 & 0.18 \\
\hline 33.3 & 0.18 & 0.18 & 0.18 & 0.18 & 0.18 & 0.18 \\
\hline 13.3 & 0.60 & 0.46 & 0.37 & 0.33 & 0.30 & 0.28 \\
\hline 10 & 0.66 & 0.49 & 0.37 & 0.33 & 0.29 & 0.27 \\
\hline 5 & 0.60 & 0.44 & 0.33 & 0.30 & 0.26 & 0.24 \\
\hline 3.3 & 0.48 & 0.36 & 0.27 & 0.24 & 0.21 & 0.19 \\
\hline 2 & 0.32 & 0.24 & 0.18 & 0.16 & 0.14 & 0.13 \\
\hline 1 & 0.19 & 0.14 & 0.11 & 0.10 & 0.09 & 0.08 \\
\hline 0.5 & 0.14 & 0.11 & 0.08 & 0.07 & 0.07 & 0.06 \\
\hline 0.25 & 0.06 & 0.05 & 0.04 & 0.03 & 0.03 & 0.03 \\
\hline
\end{tabular}


HNF-SD-W314-PDS-002, Rev. 3

Table 3-5 Response Level

\begin{tabular}{|c|c|}
\hline & Damping ( $\%$ of critical) \\
\hline & Response Level 2 \\
\hline Demand/capacity ratio & $\sim 0.5$ to 1.0 \\
\hline Welded and friction bolted metal structures & 4 \\
\hline Bearing-bolted metal structures & 7 \\
\hline $\begin{array}{l}\text { Prestressed concrete structures without } \\
\text { complete loss of prestress }\end{array}$ & 5 \\
\hline Reinforced concrete structures & 7 \\
\hline Masonry shear walls & 7 \\
\hline Wood structures with nailed joints & 10 \\
\hline Distribution systems & 5 \\
\hline $\begin{array}{l}\text { Massive low stressed components (pumps, } \\
\text { motors, etc.) }\end{array}$ & 3 \\
\hline Light welded instrument racks & 3 \\
\hline Electrical cabinets and other equipment & 4 \\
\hline $\begin{array}{l}\text { Liquid containing metal tanks } \\
\text { Impulsive mode }\end{array}$ & 3 \\
\hline $\begin{array}{l}\text { Liquid containing metal tanks } \\
\text { Sloshing mode }\end{array}$ & 0.5 \\
\hline
\end{tabular}

3.2.5.1.4 Wind Loads. For PC3 systems:

"Fastest Mile" wind velocity: $\quad 36 \mathrm{~m} / \mathrm{s}(80 \mathrm{mi} / \mathrm{h})$

"Three Second Gust" wind velocity: $44 \mathrm{~m} / \mathrm{s}(100 \mathrm{mi} / \mathrm{h})$

Missile (horizontal): $\quad 50 \times 100 \mathrm{~mm}(2 \times 4 \mathrm{in}$.) Timber plank weighing 7 $\mathrm{kg}(15 \mathrm{lb}) @ 22 \mathrm{~m} / \mathrm{s}(50 \mathrm{mi} / \mathrm{h})$. Maximum trajectory height $=9 \mathrm{~m}(30 \mathrm{ft})$.

For PC1 systems:

"Fastest Mile" wind velocity: $\quad 31 \mathrm{~m} / \mathrm{s}(70 \mathrm{mi} / \mathrm{h})$

"Three Second Gust" wind velocity: $38 \mathrm{~m} / \mathrm{s}(85 \mathrm{mi} / \mathrm{h})$

3.2.5.1.5 Snow Loads. The ground snow loads are: $720 \mathrm{~Pa}(15 \mathrm{lb} / \mathrm{square}$ foot)

3.2.5.1.6 Relative Humidity. The relative humidity range is 0 to $100 \%$ (Rate of change is negligible).

3.2.5.1.7 Surface Precipitation. The surface precipitation is $4 \mathrm{~cm}(1.56 \mathrm{in})$ in a 24 hour period.

3.2.5.1.8 Hail Events. The hail diameter is less than or equal to $1.9 \mathrm{~cm}(0.75 \mathrm{in})$. 
3.2.5.1.9 Sand and Dust. The sand/dust concentration is $0.177 \mathrm{gm} /$ cubic meter with a typical size of $350 \mu \mathrm{m}$.

3.2.5.1.10 Solar Radiation. The solar radiation range is between 4 Watts/square meter and 406 Watts/square meter.

3.2.5.1.11 Glaze. (See definition is Section 5.1) The glaze is $2.54 \mathrm{~cm}(1 \mathrm{in}$.).

3.2.5.1.12 Ashfall. A total combination load of snow fall and ash fall of $960 \mathrm{~Pa}(20 \mathrm{lb} / \mathrm{square}$ foot) shall be used.

3.2.5.1.13 Dead Loads. Dead loads include the weights of all permanent materials and equipment, including the structure's own weight. Design dead loads shall include the weight of all permanent service equipment. Load calculations shall include an allowance for any loadings anticipated to be added at a later date. Initially assumed loads shall be revised so that the final design reflects the configuration shown on the drawings.

The unit weights of materials and construction assemblies for buildings and other structures shall be those given in ASCE 7-95. Where unit weights are neither established in that standard nor determined by test or analysis, the weights shall be determined from data in manufacturer drawings or catalogs.

3.2.5.1.14 Thermal Forces. The design of structures shall include the effects of stresses and movements resulting from variations in temperature. Structures shall be designed for movements resulting from the maximum seasonal temperature change. The design shall provide for the lags between air temperatures and the interior temperatures of massive concrete members or structures. Consideration shall be given to passive soil loading resulting from thermal growth of subgrade structures.

3.2.5.1.15 Creep and Shrinkage Forces. Concrete and masonry structures shall be investigated for stresses and deformations induced by creep and shrinkage. For concrete and masonry structures, the minimum linear coefficient of shrinkage shall be assumed to be 0.0002 $\mathrm{mm} / \mathrm{mm}$, unless a detailed analysis is undertaken. The theoretical shrinkage displacement shall be computed as the product of the linear coefficient and the length of the member.

3.2.5.1.16 Load Combinations and Allowable Stresses. Load combinations, allowable stresses, and strength requirements for load conditions that include live load, dead load, snow load, and normal operating loads for all SSCs shall comply with the UBC or applicable system national codes and standards.

Load combinations, allowable stresses, and strength requirements for load conditions that include live load; dead load; snow load; normal operating loads; and NPH loads of extreme wind, earthquake, flood, and ashfall for all SSCs shall comply with DOE-STD-1020.

Load factors and load combinations for PC1 structures, systems, and components

Combine response from various loadings to determine the structural demand by using the UBC-specified load combination rules (e.g., load factors for ultimate strength design and unit load factors for allowable stress design). 
Where:

$$
\begin{aligned}
& \mathrm{DL}=\text { DEAD LOAD } \\
& \mathrm{LL}=\text { LIVE LOAD } \\
& \mathrm{RL}=\text { ROOF LIVE LOAD } \\
& \mathrm{S}=\text { SNOW LOAD } \\
& W=\text { WIND LOAD } \\
& \mathrm{E}=\text { EARTHQUAKE } \\
& \mathrm{T}=\text { THERMAL } \\
& \mathrm{C}=\text { CREEP/SHRINKAGE }
\end{aligned}
$$

Allowable Stress (AS) Design: All Construction

$A S=D L+L L+R L$ (or $S$ )

$A S=0.75[D L+L L+W($ or $E)]$

$A S=0.75[D L+L L+W+S / 2]$

$A S=0.75[D L+L L+S+W / 2]$

Ultimate Strength (U) Design: Reinforced Concrete

$U=1.4 \mathrm{DL}+1.7 \mathrm{LL}+1.7 \mathrm{RL}$ (or $1.7 \mathrm{~S}$ )

$U=0.75[1.4 \mathrm{DL}+1.7 \mathrm{LL}+1.7 \mathrm{~W}]$

$U=1.05[D L+L L+E]$

$U=0.9 D L+1.3 W$ (or $1.4 E)$

$U=1.4[D L+T($ or $C)]$

$U=0.75[1.4 \mathrm{DL}+1.7 \mathrm{LL}+1.4 T$ (or $1.4 \mathrm{C})]$

Strength (S) Design: Steel (Load \& Resistance Factor Design) $\quad S=1.4 \mathrm{DL}$

$S=1.2 \mathrm{DL}+1.6 \mathrm{LL}+0.5(\mathrm{RL}$ or $\mathrm{S})$

$\mathrm{S}=1.2 \mathrm{DL}+0.5 \mathrm{LL}$ (or $0.8 \mathrm{~W})+1.6(\mathrm{RL}$ or $\mathrm{S})$

$S=1.2 \mathrm{DL}+1.3 \mathrm{~W}+0.5 \mathrm{LL}+0.5(\mathrm{RL}$ or $\mathrm{S})$

$S=1.2 \mathrm{DL}+1.5 \mathrm{E}+0.5 \mathrm{LL}$ (or .2S)

$S=0.9 D L-1.3 W$ (or $1.5 E$ )

Load factors and load combination for PC3 structures, systems, and components

Combine response from various loadings to determine structural demand as follows:

Where:

$\mathrm{DL}=\mathrm{DEAD} L O A D$

$L L=$ LIVE LOAD

$\mathrm{RL}=$ ROOF LIVE LOAD

$S=$ SNOW LOAD

$W=$ WIND LOAD

$E=$ EARTHQUAKE

$A=$ ASHFALL

$T=$ THERMAL

$\mathrm{C}=$ CREEP/SHRINKAGE

Allowable Stress (AS) Design: 


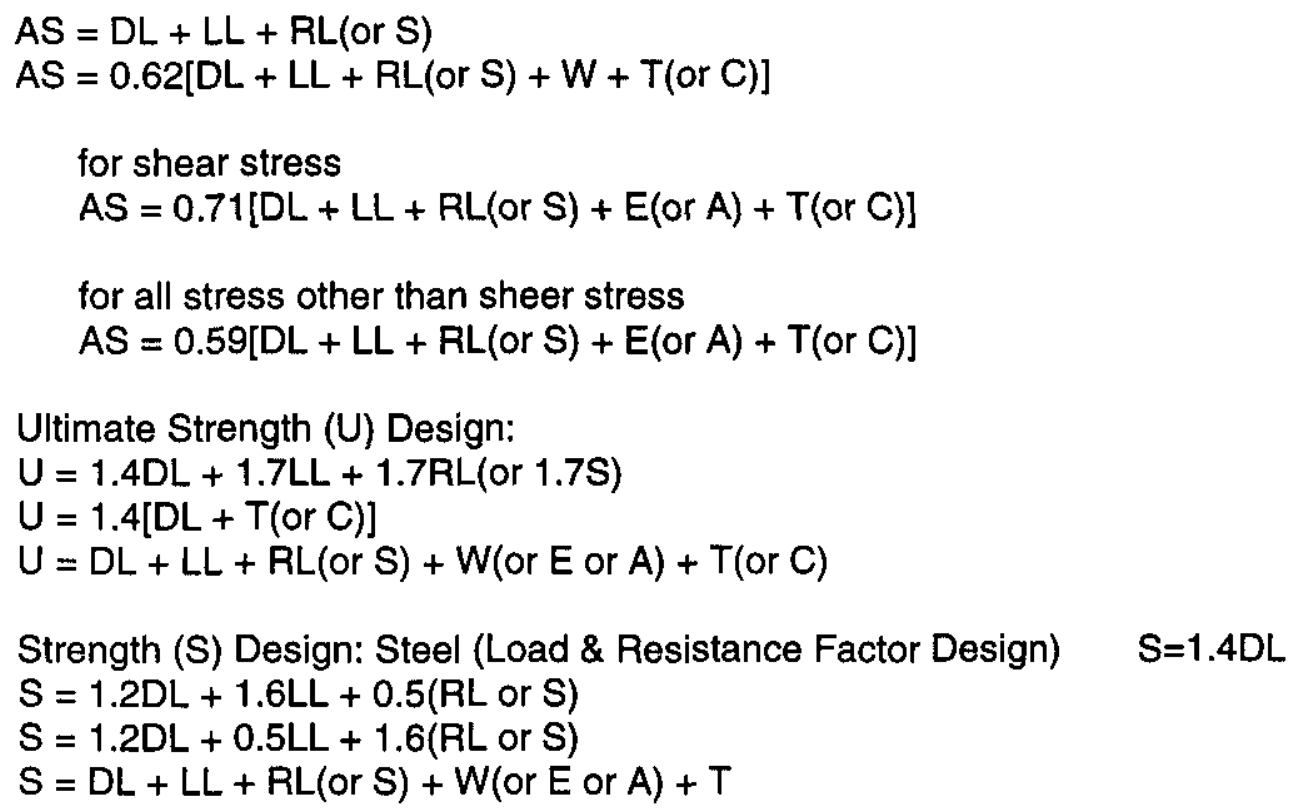

Strength (S) Design: Steel (Load \& Resistance Factor Design) $\quad S=1.4 D L$

$S=1.2 D L+1.6 L L+0.5(R L$ or $S)$

$S=1.2 \mathrm{DL}+0.5 \mathrm{LL}+1.6(\mathrm{RL}$ or $\mathrm{S})$

$S=D L+L L+R L($ or $S)+W($ or $E$ or $A)+T$

\subsubsection{Induced Environments}

3.2.5.2.1 Waste Properties. Materials used that come in contact with the waste shall be capable of safely handling waste with the following properties:

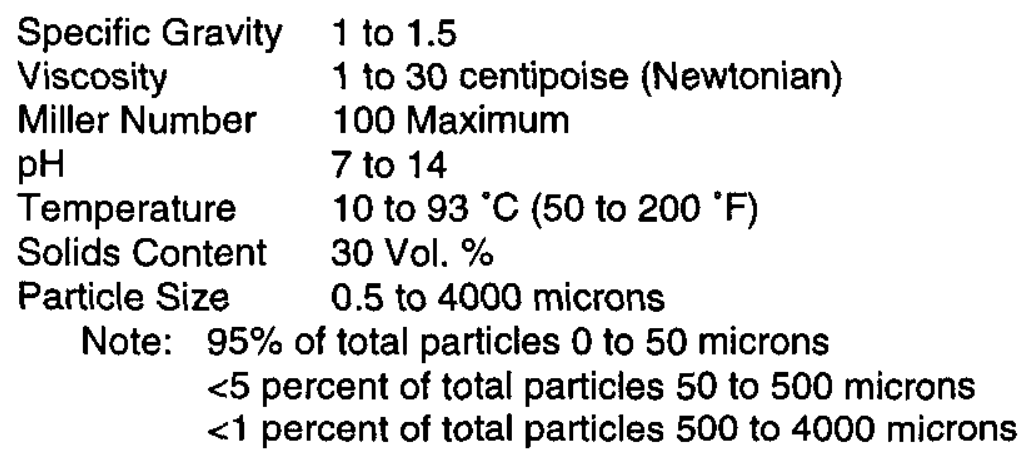

\subsection{Radiation Tolerance}

3.2.5.2.1 Inside Pit Radiation Level. Materials used that are located inside a pit shall be capable of operating in the following radiation environment:

total accumulated dose: $\quad 6.0 \mathrm{E}+7$ rads dose rate: $1.0 \mathrm{E}+7 \mathrm{mr} / \mathrm{hr}$

3.2.5.2.2 Background Pit Radiation Level. Materials used that are located outside a pit shall be capable of operating in the following radiation environment:

total accumulated dose: dose rate:

$4.4 \mathrm{rad} /$ year $0.5 \mathrm{mrem} / \mathrm{hour}$

\subsubsection{Transportability}


Not applicable to this specification.

\subsubsection{Flexibility and Expansion}

Each system design shall, to the maximum extent practicable, provide sufficient flexibility to accommodate for programmatic changes or operation modifications.

\subsection{Design and Construction}

\subsubsection{Materials, Processes, and Parts}

3.3.1.1 Design and Construction Requirements. The valve piping system shall meet the applicable design and construction requirements contained in DOE Order 6430.1A, HS-BS-0084, ASME B31.3, and UCRL-15910.

3.3.1.2 Cover Blocks Design and Construction Requirements. The new pits and cover blocks shall meet the applicable design and construction requirements contained in DOE Order 6430.1 A, ACl-318, ACl-349, UCRL-15910, AISC, and ASCE 7.

3.3.1.3 Valve Position Sensor System. The valve position sensor systems shall comply with NFPA 70 in accordance with DOE Order 6430.1A, Section 1600.

3.3.1.4 Electrical Materials and Equipment. Electrical materials and equipment shall be UL or FM tested, with label attached, for the purpose intended, whenever such products are available. Where there are no UL or FM listed products of the type, testing and certification by another nationally recognized testing agency may be acceptable.

3.3.1.5 Field Control Equipment. Field control equipment shall comply with the NEMA ICS standards and UL 508A in accordance with DOE Order 6430.1A, Section 1640-2.5.

3.3.1.6 Corrosion Resistance. All components of the valve manifold piping system including manifold supports, but excluding the PUREX connector assemblies, should be exterior corrosion resistant (e.g., coated with suitable corrosion resistant materials).

3.3.1.7 Two-Way Full Ported Valves. Two-way valves shall be full ported and designed for isolation service in accordance with ANSI B16.34.

3.3.1.8 Three-Way Full Ported Valves. Three-way valves shall be full ported, designed in accordance with ANSI B16.34 and seat closure tested per MSS-SP-61 or API 598 . For seat closure test there shall be no leakage from the flow side to the isolated port for each flow position.

3.3.1.9 Optimization. During the design of facilities, optimization principles, as discussed in ICRP Publication 37, shall be utilized in developing and justifying facility design and physical controls.

\subsubsection{Electromagnetic Radiation}

3.3.2.1 Interference. Hand held radio and cell phone wattage frequency shall not interfere with 
electrical/electronic components at a distance of one meter.

3.3.2.2 Electromagnetic Force Interference. The valve position system shall not be adversely affected by outside electromagnetic forces generated by a 60 horse power, $480 \mathrm{Vac}$, induction type motor operating at frequencies between 0 and 60 hertz and located 1 meter away from the leak detection system.

\subsubsection{Identification and Marking}

3.3.3.1 Labeling. New equipment and/or modifications to existing equipment shall be labeled in a standardized format in accordance with HNF-IP-0842, Volume II, Section 6.1, Rev. 0.

3.3.3.2 Master Equipment List. A master equipment list consisting of electronic database for structures, systems, and components shall be generated in accordance with HNF-IP-0842, Volume II, Section 6.1, Rev. 0. The list contains such information as: unique equipment label, drawing reference, procedures, vendor information, safety classification, nameplate data, etc.

3.3.3.3 Operations and Maintenance Drawings. Operations and maintenance drawings (as-built $\mathrm{H}-14$ essential drawings) shall be prepared as the system master drawings showing as-built configuration changes in accordance with HNF-IP-0842, Volume II, Section 6.1, Rev. 0.

3.3.3.4 Valve Extension Handle Identification. Valve extension handles shall be uniquely identified.

\subsubsection{Workmanship}

3.3.4.1 Valve Pit Piping Workmanship. The design of the valve piping system shall determine and specify appropriate levels of workmanship for the fabrication and installation of the manifold according to the requirements of ASME B31.3, except PUREX connectors.

3.3.4.2 Cover Blocks and New Pits Workmanship. The design of the cover blocks and new pits shall determine and specify appropriate levels of workmanship for the fabrication and installation of the cover blocks and new pits according to the requirements of $\mathrm{ACl}-301$, Section 1 through 5 and $\mathrm{ACl}-318$, Part 3.

\subsubsection{Interchangeability}

All like equipment (valves, position sensors, etc.) shall have interchangeable parts.

\subsubsection{Safety}

\subsubsection{Nuclear Safety}

3.3.6.1.1 Safety Classification. The cover blocks and new pits are identified as safety class items for the structures, systems, and components (SSCs). The safety class function of the cover blocks and new pits is to knockdown spray and limit the release of aerosols to the atmosphere.

3.3.6.1.2 Flammable Gas Hazardous Environment. The components of the valve position sensor systems installed in the tank intrusive locations, shall be intrinsically safe, designed to 
meet NFPA 70 Class I, Division 1, Group B criteria for hazardous locations.

\subsubsection{Human Performance/Human Engineering}

3.3.7.1 Control Devices. Each control device shall be in accordance with NUREG 0700, Section 6.4 and MIL-STD-1472E, Section 5.4 in accordance with DOE 6430.1A, Section 1300-12.4.7.

3.3.7.2 Display Devices. Each display device shall be in accordance with NUREG 0700, Section 6.5 and MIL-STD-1472E, Section 5.2 in accordance with DOE 6430.1A, Section 1300-12.4.6.

3.3.7.3 Valve Actuator Handwheel. Each valve actuator shall be designed to produce the required operating torque with a maximum rim pull of $80 \mathrm{lbs}$ on the handwheel.

\subsection{Documentation}

\subsubsection{Document Control}

Records, documents, and document control pertinent to design functions shall be in accordance with RPP-PRO-224 and RPP-PRO-233.

\subsubsection{Drawings Preparation}

Drawings shall be prepared according to the formats set forth in HNF-PRO-242, Rev. 1, Engineering Drawing Requirements for Documents produced prior to June 15, 1999 and RPP-PRO-709, Rev. 0, Preparation and Control Standards for Engineering Drawings produced after June 15, 1999.

\subsection{Logistics}

\subsubsection{Maintenance}

3.5.1.1 Fully Remote Maintenance and Operation. Each system or portion of a system having radiation levels greater than $50 \mathrm{mrem} / \mathrm{hr}$ contact exposure shall be designed to be remotely maintained and operated or designed to require no maintenance and be remotely operated.

3.5.1.2 Limited Contact Malntenance and Operation. Each system or portion of a system having radiation levels greater than $0.1 \mathrm{mrem} / \mathrm{hr}$ to less than or equal to $50 \mathrm{mrem} / \mathrm{hr}$ shall be designed for limited contact maintenance and operation.

3.5.1.3 Full Contact Maintenance and Operation. Each system or portion of a system having radiation levels less than or equal to $0.1 \mathrm{mrem} / \mathrm{hr}$ shall be designed for full contact maintenance and operation.

3.5.1.4 Dome Loading Constraints. The equipment used for installation and maintenance must comply with the DST dome loading constraints.

3.5.1.5 Valve Maintenance. Valves shall be maintenance free with up to 1000 cycles over a 
design life of 12 years.

\subsubsection{Supply}

3.5.2.1 Parts and Components. The system design shall, to the greatest extent practicable, use readily available parts and components.

\subsubsection{Facilities and Facility Equipment}

New Hanford Site remote operation wrenches shall be developed for installation/removal of the PUREX Jumper Connector Assembly.

\subsection{Personnel and Training}

The valve pit piping system, cover blocks, and valve position sensor systems, shall be designed for operation by personnel possessing qualifications in accordance with DOE 5480.20A, Chapter IV, and trained in accordance with Chapter 1.

\subsection{Major Component Characteristics}

\subsubsection{New Process Pits and Cover Blocks}

\subsubsection{Performance Characteristics}

3.7.1.1.1 Confine Waste Leakage within the Transfer Associated Structures. (FFBD Function 3.2.1)

3.7.1.1.1.1 Transfer Line Equipment. The process pit(s) shall support transfer line equipment located in and above the pits, nozzles where transfer lines terminate at the pit sidewalls, and the cover blocks.

3.7.1.1.1.2 Equipment in Process Pits. The process pit(s) and cover blocks shall enclose the valve pit piping system and pit leak detection equipment.

3.7.1.1.1.3 Process Pit Liner. The process pit(s) with liners/special protective coatings shall provide leaktight secondary confinement.

3.7.1.1.1.4 Release of Contamination. The process pit(s) and cover blocks shall confine release of any aerosol spray of a product leak from the valve manifold within the valve or pump pit. Compressible gasket shall be used between cover block and top of pit to prevent leakage.

3.7.1.1.1.5 Leak Detection Equipment Location. The process pit(s) shall provide a low-point location in the floor for the pit leak detection equipment.

3.7.1.1.1.6 Low-Point Drain System. The process pit(s) shall provide a low-point drain system to return accumulated liquids, leakage from the primary confinement system, and wash-down liquids to a Double-Shell Tank.

3.7.1.1.1.7 Radiation Shielding. The process pit(s) and cover blocks, including penetrations, shall provide adequate shielding for facility workers located on top of the cover blocks and 
adjacent to the process pits during normal operations and maintenance. The radiation shielding shall meet the design objective to maintain exposure levels below an average of $0.5 \mathrm{mrem}$ ( 5 microsieverts) per hour and as far below this average as is reasonably achievable. The goal for reasonably achievable is 0.05 mrem ( 5 microsieverts) per hour or less.

3.7.1.1.1.8 Cover Block Rain Intrusion. The cover blocks shall be designed to limit intrusion of rain water or snow melt into the process pits.

3.7.1.1.1.9 Protective Coating. New process pit(s) shall be equipped with stainless steel liners that extend to the bottom of the cover blocks. All other exposed surfaces and the cover blocks shall have a special protective coating to protect the structural secondary confinement by providing a barrier between the interior exposed surfaces of the pit and cover blocks and any waste present in the pit due to leaks, spill, or intrusion. 241-AN-04D Slurry Receiver Pit shall be protected by SPC on exposed surfaces.

3.7.1.1.1.10 Low-Point Drain System Capabilities. The drain system shall have the ability to drain accumulated leakage within 24 hours.

\subsubsection{Physical Characteristics}

3.7.1.2.1 Reinforced Concrete. New process pits and cover blocks shall be constructed of reinforced concrete and pits shall be below grade. Edges of the cover blocks shall be stepped to preclude radiation streaming.

3.7.1.2.2 Process Pit Wall Nozzles. Process pits shall be equipped with sleeves for wall nozzles as required. Wall nozzles shall be PUREX type design (reference drawings $\mathrm{H}-2-90185$ and $\mathrm{H}-2-90186)$.

3.7.1.2.3 Process Pit Spare Nozzles. Sleeves for spare nozzles shall be installed in any new process pit, except for the new 241-AN-04D Slurry Receiver Pit.

3.7.1.2.4 Grounding Systems. A grounding system shall be furnished for electrical equipment, structural components, valve pit liners, reinforcing steel, nozzles and other metallic equipment. This grounding system shall be interfaced to the appropriate tank farm grounding and cathodic protection systems.

3.7.1.2.5 Cover Blocks Penetrations. New cover blocks and existing cover blocks to be modified (see 3.1) shall be equipped with penetrations to facilitate operations and/or maintenance of manifold valves, pit drains, pit leak detection devices and transfer leak detection devices. Additionally, the penetrations shall provide for access to any other equipment in the pits requiring maintenance, pit wash down, gas sampling ports, and installation of support equipment (e.g., video cameras, etc.). A sufficient number of penetrations shall be provided to allow full visual inspection of the interior of the pits.

3.7.1.2.6 Cover Blocks Rain Ingress. Cover blocks shall be sloped to drain off the top of the blocks and shall extend over the outside of the pit walls, except that the single piece cover block for 241-AN-04D Slurry Receiver Pit shall not be sloped.

3.7.1.2.7 Cover Blocks for Pump Pits. The cover blocks for 241-AZ-02A, 241-AZ-01A, 241-AY-01A, and 241-AY-02A Central Pump Pits, including penetrations shall be sealed to limit 
air infiltrations.

3.7.1.2.8 Gas Sampling Ports. Gas sampling ports shall be greater than 0.7 inch and less than 1.0 inch in diameter, located above the drain or center of the pit, flared on the bottom to minimize snagging of sample line during removal, and equipped with two bonding lugs for attachment to the portable sampler.

3.7.1.2.9 Gas Sampling Ports Material. Gas sampling ports shall be constructed of low sparking, electrically conductive material, and shall be electrically bonded.

3.7.1.2.10 Gas Sampling Port Covers. Gas sampling ports shall have an electrically conductive, non-screw type cover that is electrically bonded to the port with a noninsulated conductor strong enough to act as a cover keeper.

3.7.1.2.11 Lifting Mechanism. Cover blocks shall be supplied with lifting mechanisms on the top surface to facilitate handling with a crane. The maximum weight of new cover block shall be 16.5 ton.

3.7.1.2.12 Cover Blocks Alignment. Cover blocks and pit walls shall be marked to facilitate alignment of the cover blocks.

\subsubsection{Valve Position Sensor Systems}

\subsubsection{Performance Characteristics}

3.7.2.1.1 Monitor Valve Position. (FFBD Function 3.1.4) After the transfer system route has been setup, the transfer system shall monitor the limit positions of the valves on the valve jumper manifolds in the transfer route. This requirement does not apply to 241-AN-04D Slurry Receiver Pit.

3.7.2.1.1.1 Indicate Valve Limit Position. The waste transfer system jumper/manifold valves shall indicate valve limit position.

3.7.2.1.1.2 Active Valve Indication. Each separate valve limit position shall be actively indicated.

3.7.2.1.1.3 Valve Position Transmission. The valve position shall be transmitted to the Master Pump Shutdown system.

3.7.2.1.1.4 Valve Position Indication Accuracy. The valve position indication system shall provide sufficient accuracy with respect to the actual valve position such that the selected flow path configuration is leak tight.

3.7.2.2 Physical Characteristics. This requirement does not apply to 214-AN-04D Slurry Receiver Pit.

3.7.2.2.1 Hardware Proximity. The valve position sensing hardware, display, and transmitter as applicable shall be able to withstand the insertion and removal, turning, and overturning of the valve actuator handle and still operate properly. 
3.7.2.2.2 Hardware Exposure to Environment. The valve position hardware, display, and transmitter, as applicable, shall be designed to perform in the environment on top of the cover block and withstand inadvertent physical abuse.

3.7.2.2.3 Hardware Removability. The valve position hardware, display, and transmitter, as applicable, shall be designed to facilitate quick mechanical/electrical disconnect for ease of cover block removal and replacement.

3.7.2.2.4 Hardware Capabilities. The valve position hardware to detect, display, and transmit valve position shall be located outside the pit.

\subsubsection{Pit Manifolds Sitework - New Pits}

\subsubsection{Performance Characteristics}

\subsection{Site Transfer Associated Structures. (FFBD Function 3.2.6)} Pits.

3.7.3.1.1.1 Site Soils. The site soils shall support the major components of the new Valve

3.7.3.1.1.2 Access Control Fencing. Access control to radiological and hazardous waste areas shall be provided by fencing consistent with existing fencing for RPP operations.

3.7.3.1.1.3 Existing Lighting Extended. The existing area lighting system at the 241-AZ tank farm shall be extended to provide an adequate level of illumination for night-time operations at the new 241-AZ-VP Valve Pit. The lighting system shall provide a minimum average illumination of $54 \mathrm{lux}(5 \mathrm{fc})$ with a maximum uniformity ratio of $5: 1$ average to minimum.

3.7.3.1.1.4 Lighting Control. Lighting control shall be furnished with a photocell override to ensure that lights are turned off during the day.

\subsubsection{Physical Characteristics}

3.7.3.2.1 Disturbed Areas Stabilized. All areas disturbed by construction shall be stabilized subsequent to construction.

3.7.3.2.2 Pole-Mounted Luminaries. Pole-mounted banks of luminaries shall be used. Fixture lowering devices shall be provided for poles over $18 \mathrm{~m}$ high. Existing electrical distribution system in the area shall be utilized to provide necessary power.

3.7.3.2.3 Luminary Glare. Luminaries shall be shielded mercury vapor, or metal halide directed to minimize upward glare.

\subsubsection{Pit Drain Seal Assemblies}

\subsubsection{Performance Characteristics}

3.7.4.1.1 Position Pit Drain Seal Assembly. (FFBD Function 3.1.8)

3.7.4.1.1.1 Twenty-four (24) Hour Holdup of Ten Gallons. The pit drain seal assembly shall 
provide a 24 hour holdup for ten gallons of liquid to activate a leak detector.

3.7.4.1.1.2 Vapor Seal. The pit drain seal assembly shall provide a vapor seal to prevent the tank vapor space pressure from offgassing into the pit and air in-leakage into the tank vapor space.

3.7.4.1.1.3 Retractable Drain Seal. The drain seal assembly shall be retractable to permit complete drainage of the pit and removable for gasket replacement.

3.7.4.1.1.4 Drainage to the Tank. The pit drain seal assembly shall provide drainage into the tank to prevent a pit overflow condition.

\subsubsection{Physical Characteristics}

3.7.4.2.1 Operation Above Cover Block. Operation, removal, and insertion of the drain seal assembly shall be from above the cover block.

3.7.4.2.2 Visible Indication of Position. Visible indication shall be provided above the cover block at the drain seal assembly position (i.e., inserted or retracted).

3.7.4.2.3 Configuration to Prevent Drain Plugging. Configuration of the drain seal assembly shall minimize the potential for drain line plugging in both the inserted and retracted positions.

3.7.4.2.4 Electrical Grounding Jumper. The drain seal operator shall be provided with an electrical grounding jumper.

3.7.4.2.5 Configuration to Prevent Inadvertent Manipulation. Configuration of the drain seal assembly operator shall prevent inadvertent manipulation of the drain seal assembly beyond the retracted position. 


\section{SYSTEM QUALIFICATION PROVISIONS}

\subsection{General}

The Project W-314 QAPP (HNF-SD-W314-QAPP-001) defines the quality assurance requirements for this project.

Table 4-1 listed verifications may be performed in conjunction with QAPP verifications. Inspections as defined in 4.2 shall be conducted during the design and development of each system to provide assurance of compliance with the requirements of this PDS.

\subsubsection{Responsibility for Inspections}

The design contractor shall be responsible for the performance of all inspections for each system developed in accordance with this PDS. Inspections shall be conducted at the contractor facilities or the facilities of his choice with the approval of the procuring authority. The procuring authority reserves the right to witness or perform the specified inspections.

\subsubsection{Special Tests and Examinations}

Not applicable to this specification.

\subsection{Quality Conformance Inspections.}

Qualification shall be performed on System hardware representative of the approved production design. Qualification of the System to assure compliance with the requirements of Section 3 shall be by examination, demonstration, test, and/or analysis, as defined herein. Test program data may be used to assure compliance with requirements.

a. Examination is an element of inspection consisting of investigation, without the use of special laboratory appliances or procedures, to determine compliance with requirements. This method is intended to be construction related and consists of examination of documents and construction activities.

b. Demonstration is an element of inspection that is limited to readily observable functional operation to determine compliance with requirements. This element of inspection does not require the use of special equipment or sophisticated instrumentation. This method is intended to be utilized for any mock-up testing.

c. Test is an element of inspection that employs technical means including (but not limited to) the evaluation of functional characteristics by use of special equipment or instrumentation, simulation techniques, and the application of established principles and procedures to determine compliance with requirements. The analysis of data derived from test is an integral part of this inspection. This method is intended to be utilized for any acceptance testing in the field.

d. Analysis is an element of inspection, taking the form of the processing of accumulated results and conclusions, intended to provide proof that verification of a requirement(s) has been accomplished. The analytical results may be comprised of a compilation of interpretation of 
HNF-SD-W314-PDS-002, Rev. 3

existing information or derived from lower level examinations, tests, demonstrations, or analyses.

The environmental capability of equipment shall be demonstrated by appropriate testing, analysis, and operating experience, or other methods that can be supported by auditable documentation, or a combination of these methods. 
HNF-SD-W314-PDS-002, Rev. 3

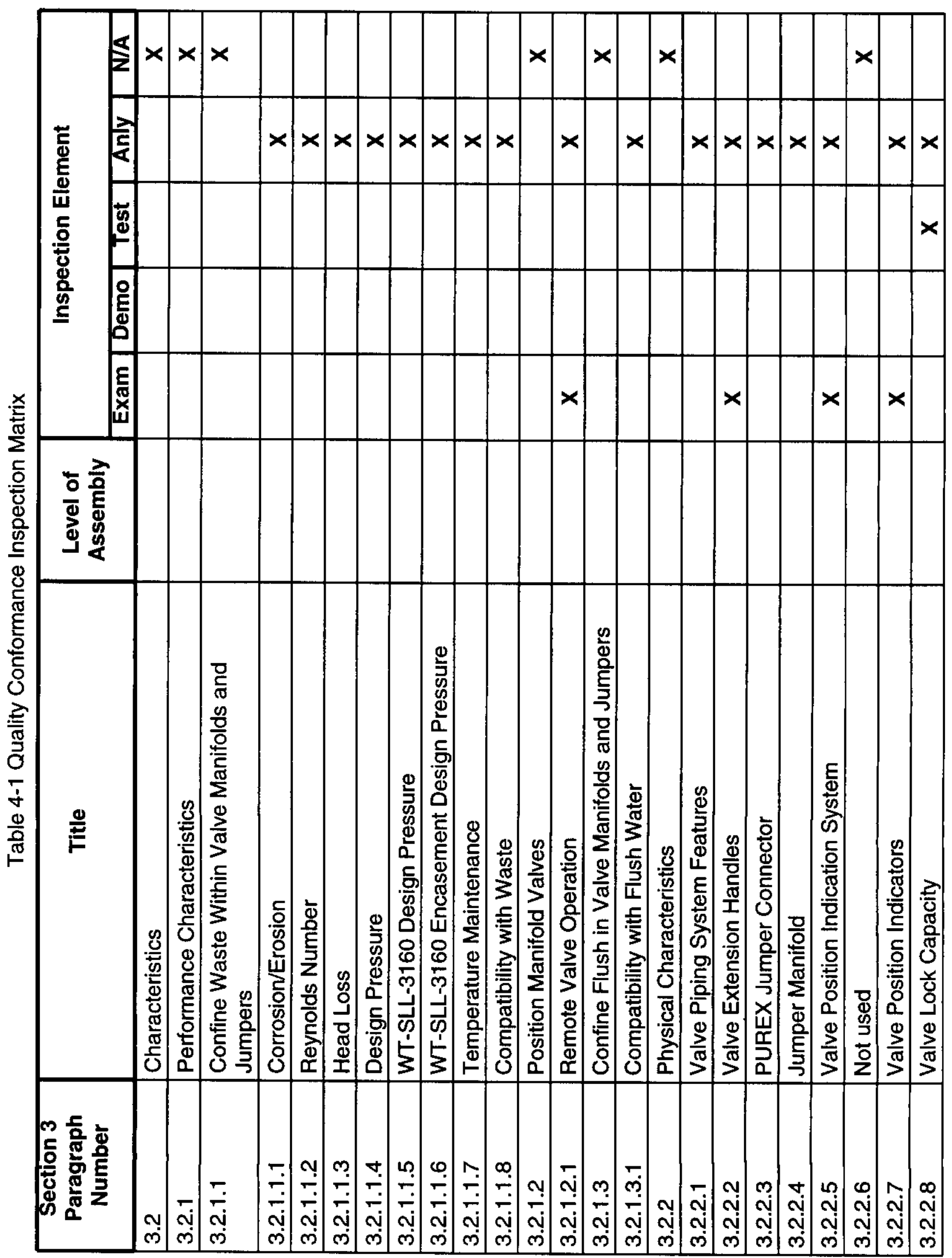


HNF-SD-W314-PDS-002, Rev. 3

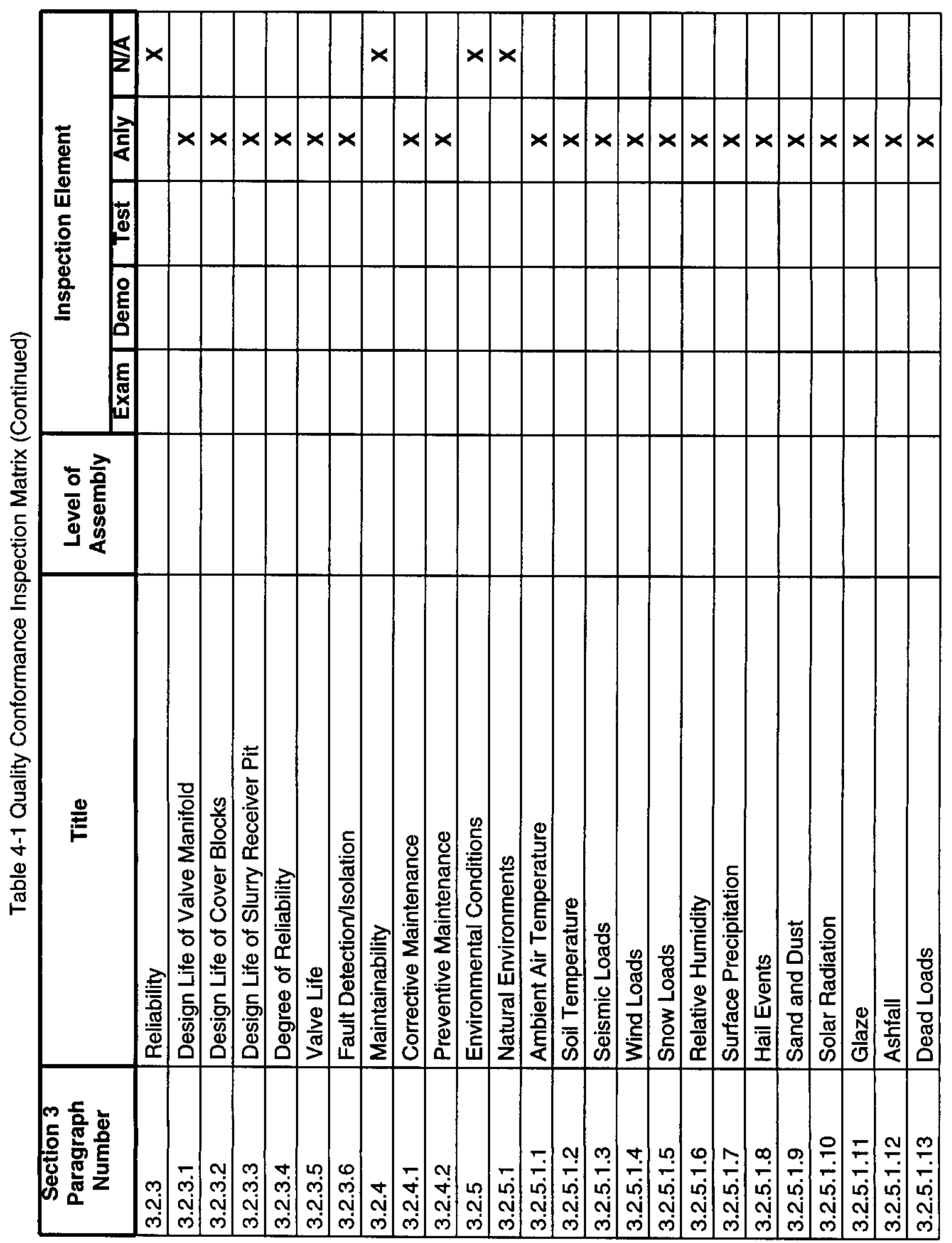


HNF-SD-W314-PDS-002, Rev. 3

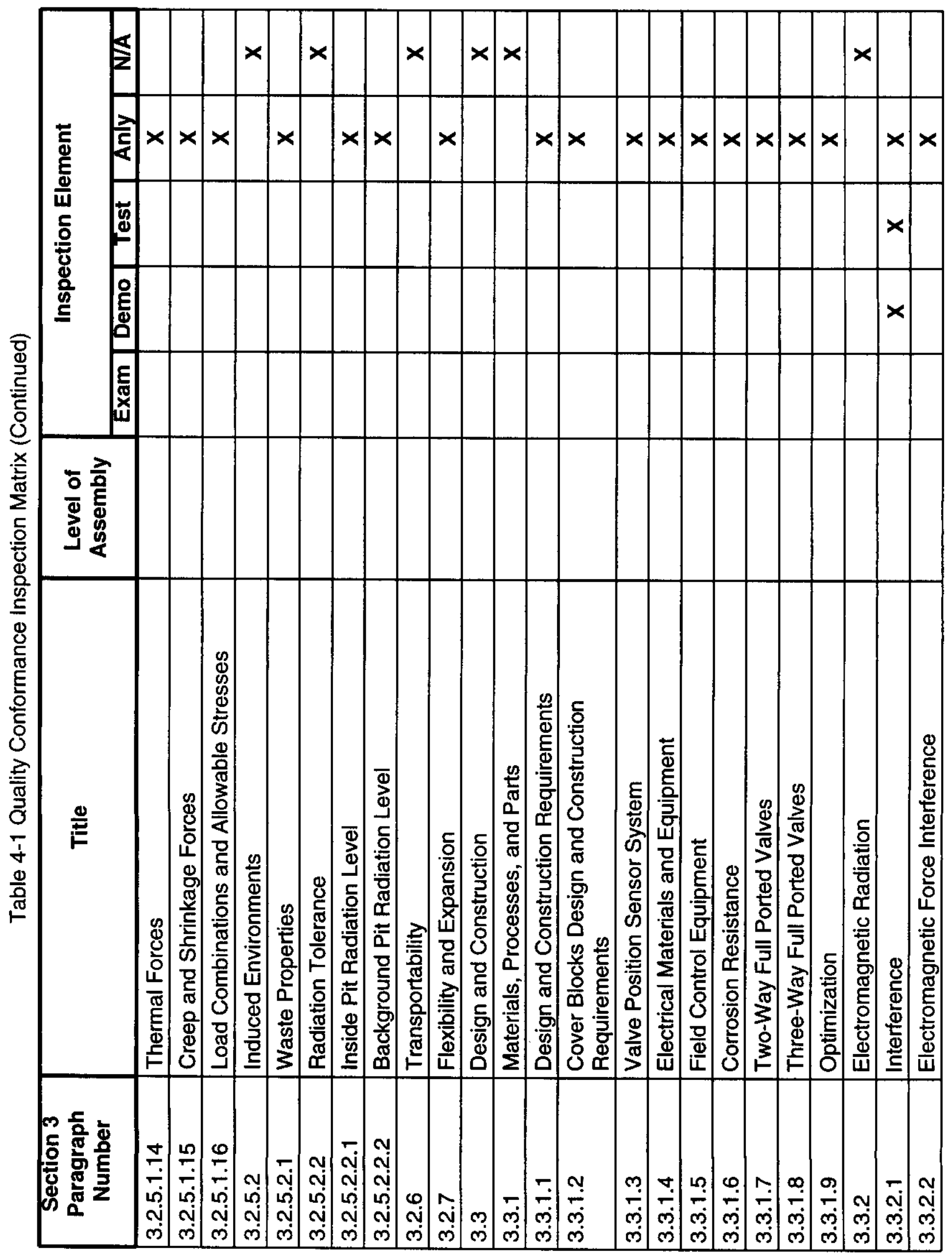


HNF-SD-W314-PDS-002, Rev. 3

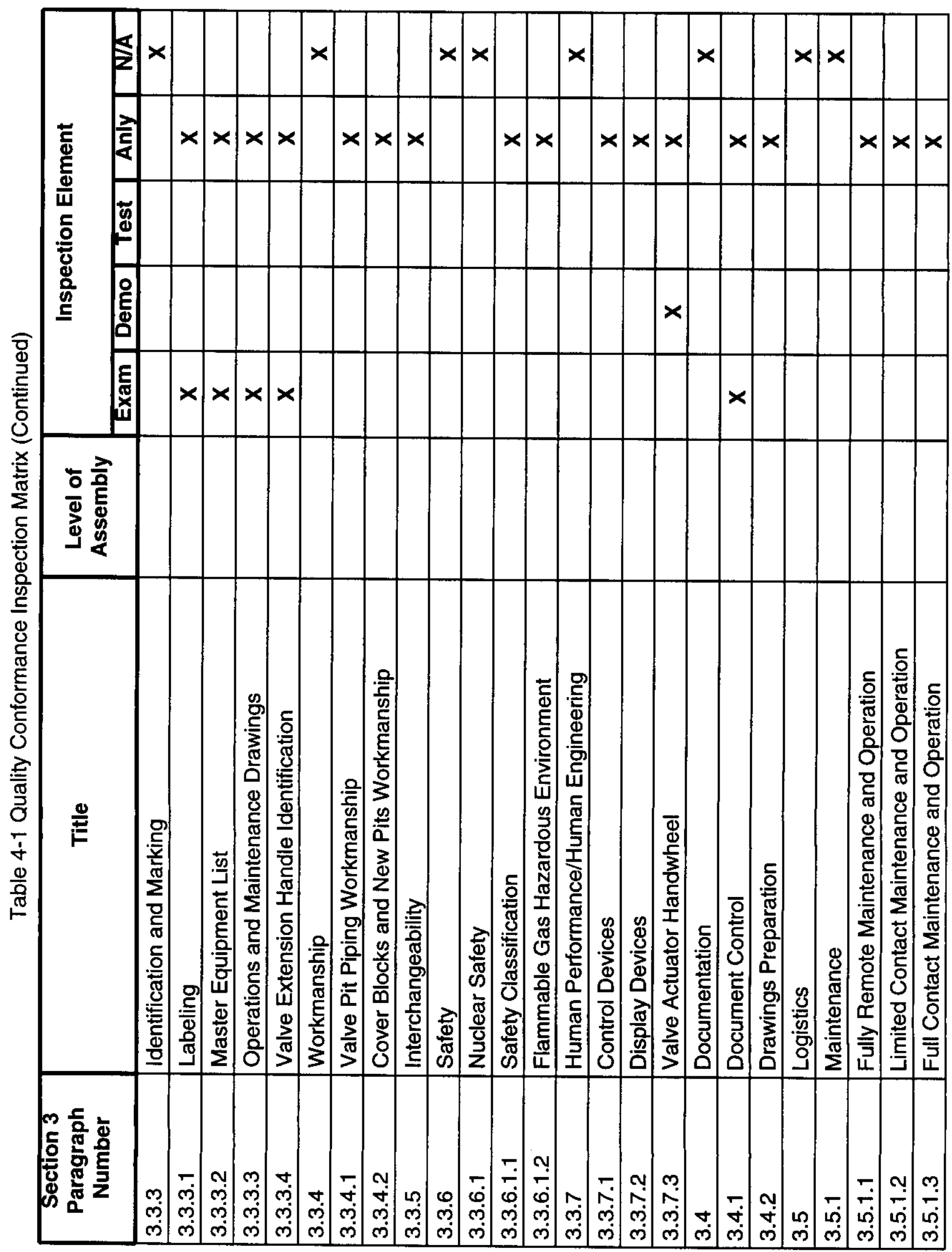


HNF-SD-W314-PDS-002, Rev. 3

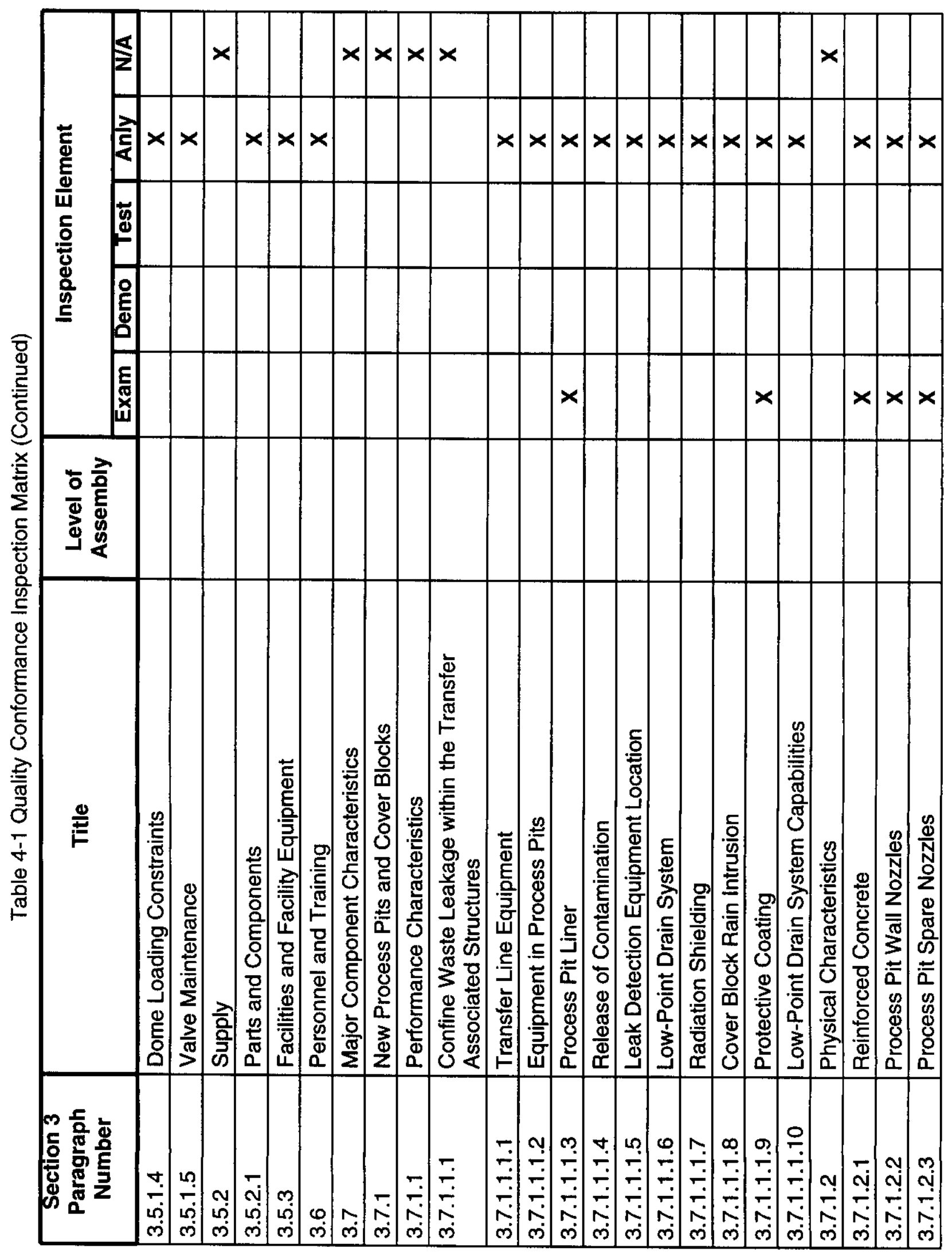


HNF-SD-W314-PDS-002, Rev. 3

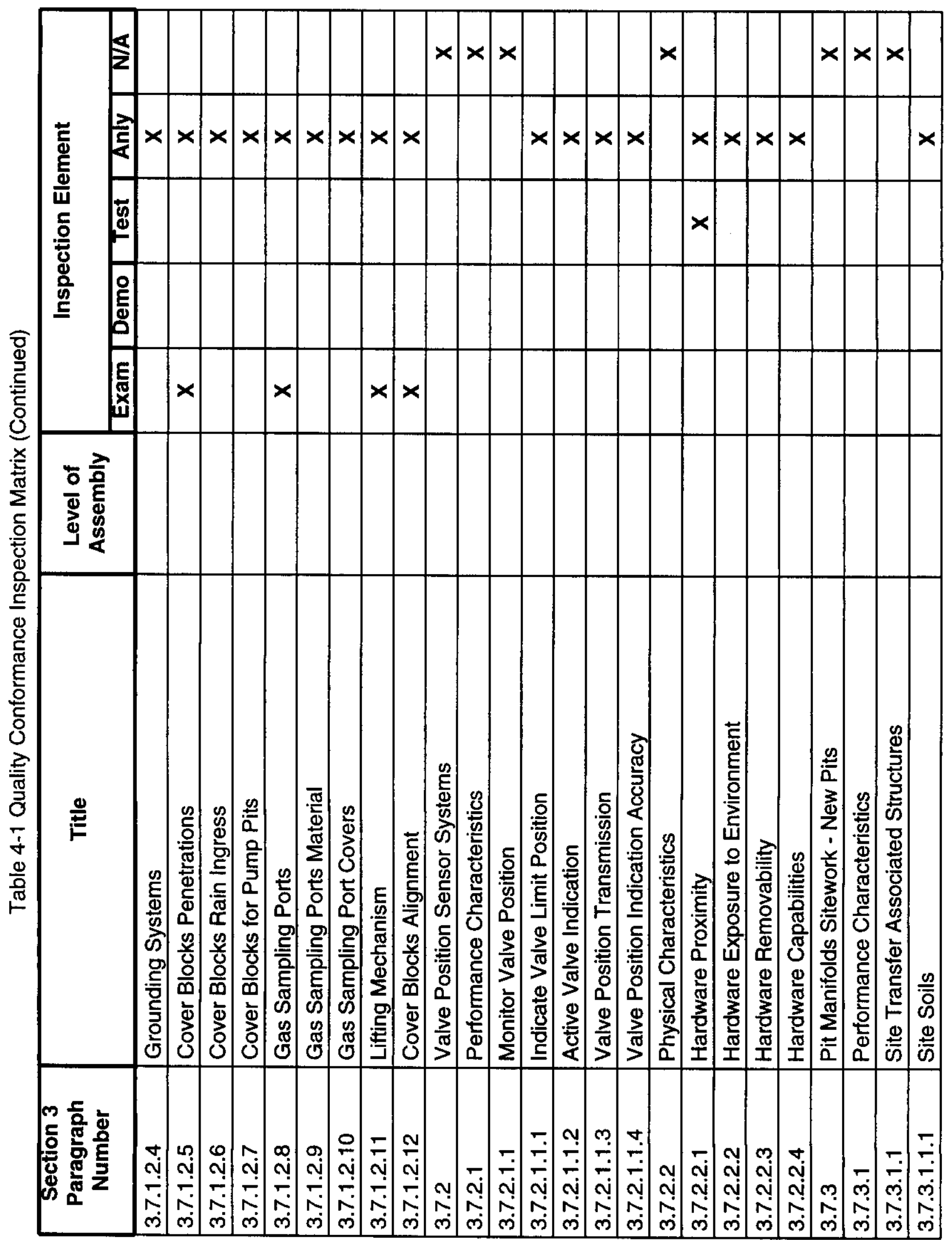


HNF-SD-W314-PDS-002, Rev. 3

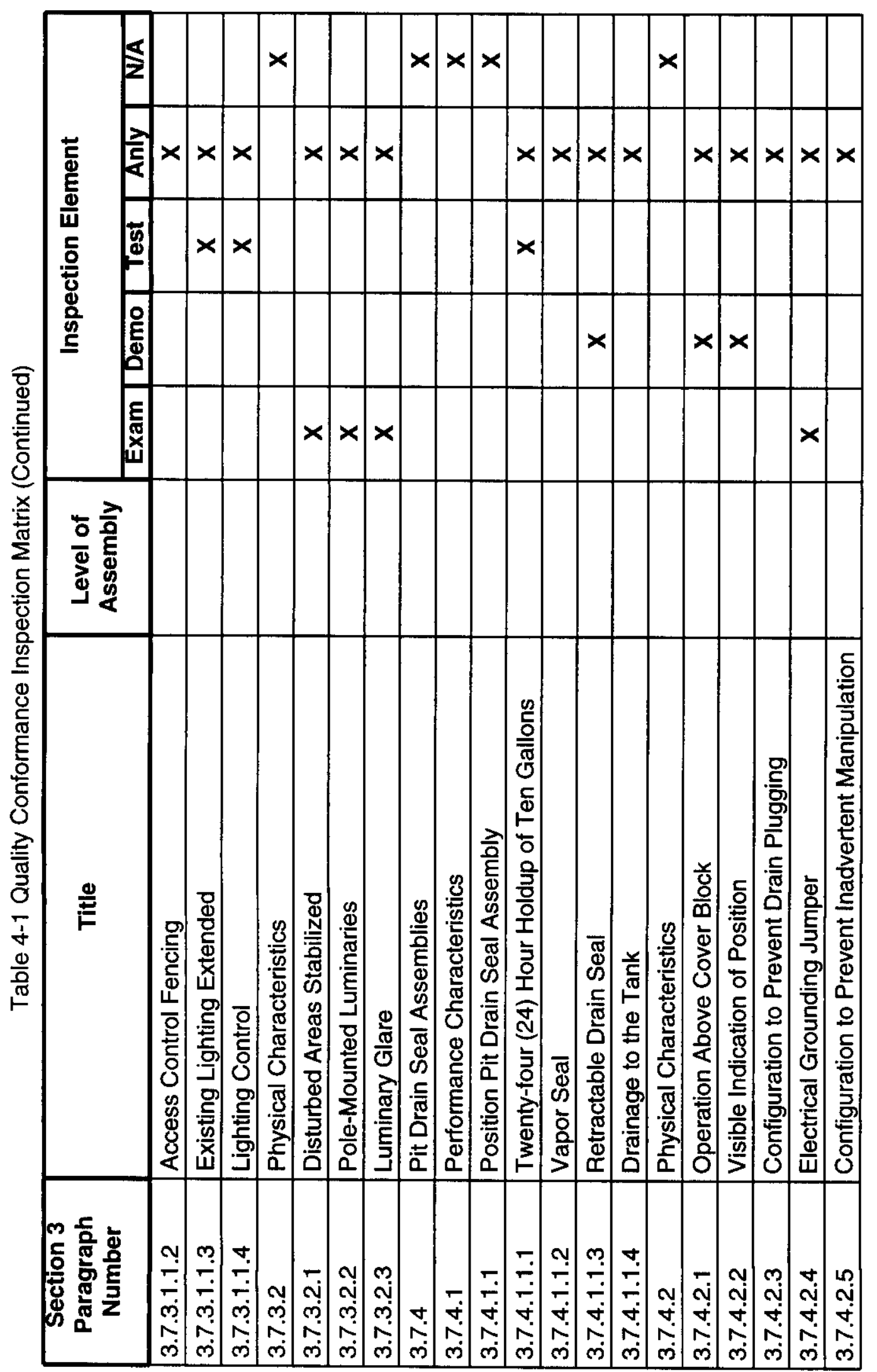




\section{NOTES}

\subsection{Definitions}

\subsubsection{Manifold}

Remotely installed rigid piping systems inside a pit that transfer waste and flush water between nozzles.

\subsubsection{Pit Nozzle}

Rigid male connector anchored in the pit wall that provides a leak tight connection with the integral seal block attached to a manifold.

\subsubsection{Equivalent Length}

The actual length of piping plus the friction loss of all valves and fittings in the system expressed in terms of equivalent feet of piping.

\subsubsection{Glaze}

Coating of ice formed when rain or drizzle freezes on contact with any surface having a temperature that is below freezing.

\subsection{Acronym List}

$\begin{array}{ll}\text { ACI } & \text { American Concrete Institute } \\ \text { AISC } & \text { American Institute of Steel Construction } \\ \text { ALARA } & \text { As Low As Reasonable Achievable } \\ \text { ANSI } & \text { American National Standard Institute } \\ \text { ASCE } & \text { American Society of Civil Engineers } \\ \text { ASME } & \text { American Society of Mechanical Engineers } \\ \text { BIO } & \text { Basis for Interim Operation } \\ \text { CDR } & \text { Conceptual Design Report } \\ \text { CFR } & \text { Code of Federal Regulations } \\ \text { DOE } & \text { Department of Energy } \\ \text { DRD } & \text { Design Requirements Document } \\ \text { DST } & \text { Double Shell Tank } \\ \text { EDTA } & \text { Ethylenediametraacetic acid } \\ \text { FDC } & \text { Functional Design Criteria } \\ \text { FFBD } & \text { Functional Flow Block Diagram } \\ \text { FM } & \text { Factory Mutual System } \\ \text { HEDTA } & \text { N-(hydroxyethyl)-ethylenediaminetriacetic acid } \\ \text { LLW } & \text { Low Level Waste } \\ \text { MTBF } & \text { Mean Time Between Failure } \\ \text { MWTF } & \text { Multi-Function Waste Tank Facility } \\ \text { N/A } & \text { Not applicable } \\ \text { NEMA } & \text { National Electrical Manufacturers Association } \\ \text { NFPA } & \text { National Fire Protection Association }\end{array}$




$\begin{array}{ll}\text { NUREG } & \text { Nuclear Regulatory Reports } \\ \text { PC } & \text { Performance Category } \\ \text { PDC } & \text { Project Design Concept } \\ \text { PDS } & \text { Project Development Specification } \\ \text { PHMC } & \text { Project Hanford Management Contract } \\ \text { PICD } & \text { Project Interface Control Document } \\ \text { PUREX } & \text { Plutonium-Uranium Extraction } \\ \text { RAS } & \text { Requirements Allocation Sheet } \\ \text { RPP } & \text { River Protection Project } \\ \text { SPC } & \text { Special Protective Coating } \\ \text { SpG } & \text { Specific Gravity } \\ \text { SST } & \text { Single Shell Tank } \\ \text { TOC } & \text { Total Organic Carbon } \\ \text { TWRS } & \text { Tank Waste Remediation System } \\ \text { UCRL } & \text { University of California Research Laboratory } \\ \text { USSR } & \text { Upgrade Scope Summary Report }\end{array}$

\subsection{Applicable Documents}

National codes and standards will be identified within Section 2, Applicable Documents, of the PDS without dates or revision numbers. Government documents and Hanford site standards will be identified by the effective date or revision number. 
HNF-SD-W314-PDS-002, Rev. 3

APPENDIX A

FUNCTIONAL FLOW BLOCK DIAGRAMS (FFBDS) 
HNF-SD-W314-PDS-002, Rev. 3
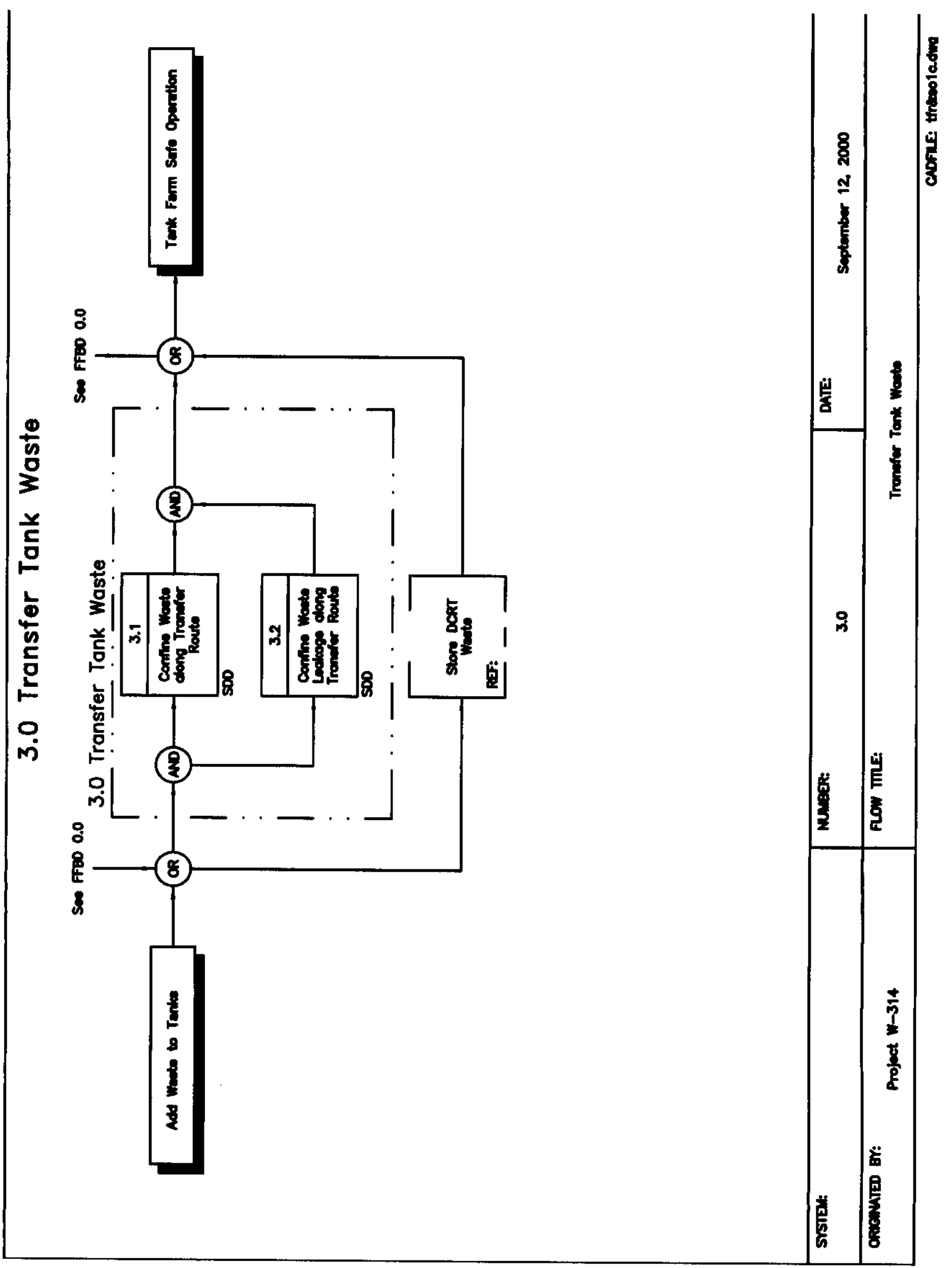
HNF-SD-W314-PDS-002, Rev. 3

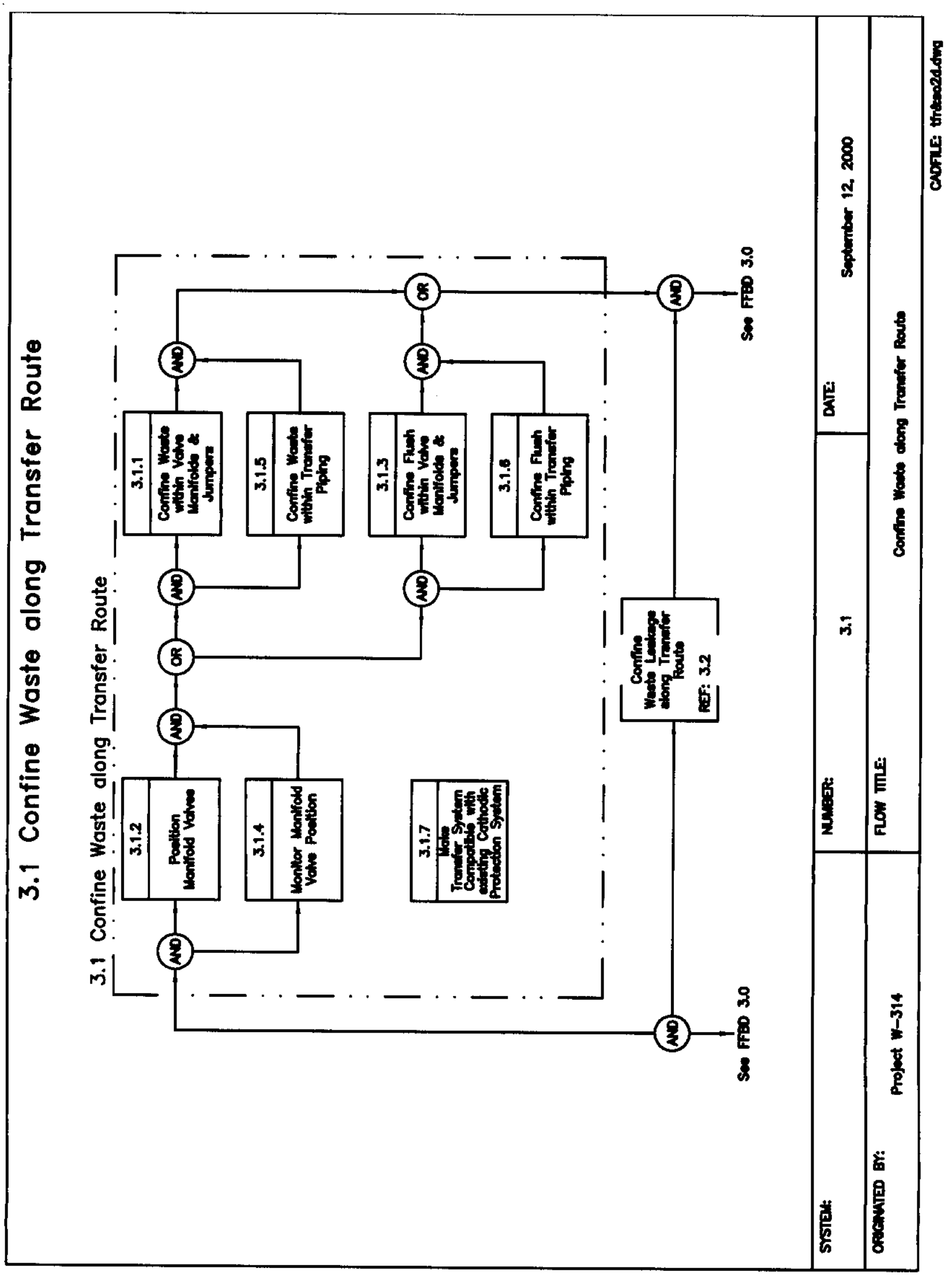


HNF-SD-W314-PDS-002, Rev. 3

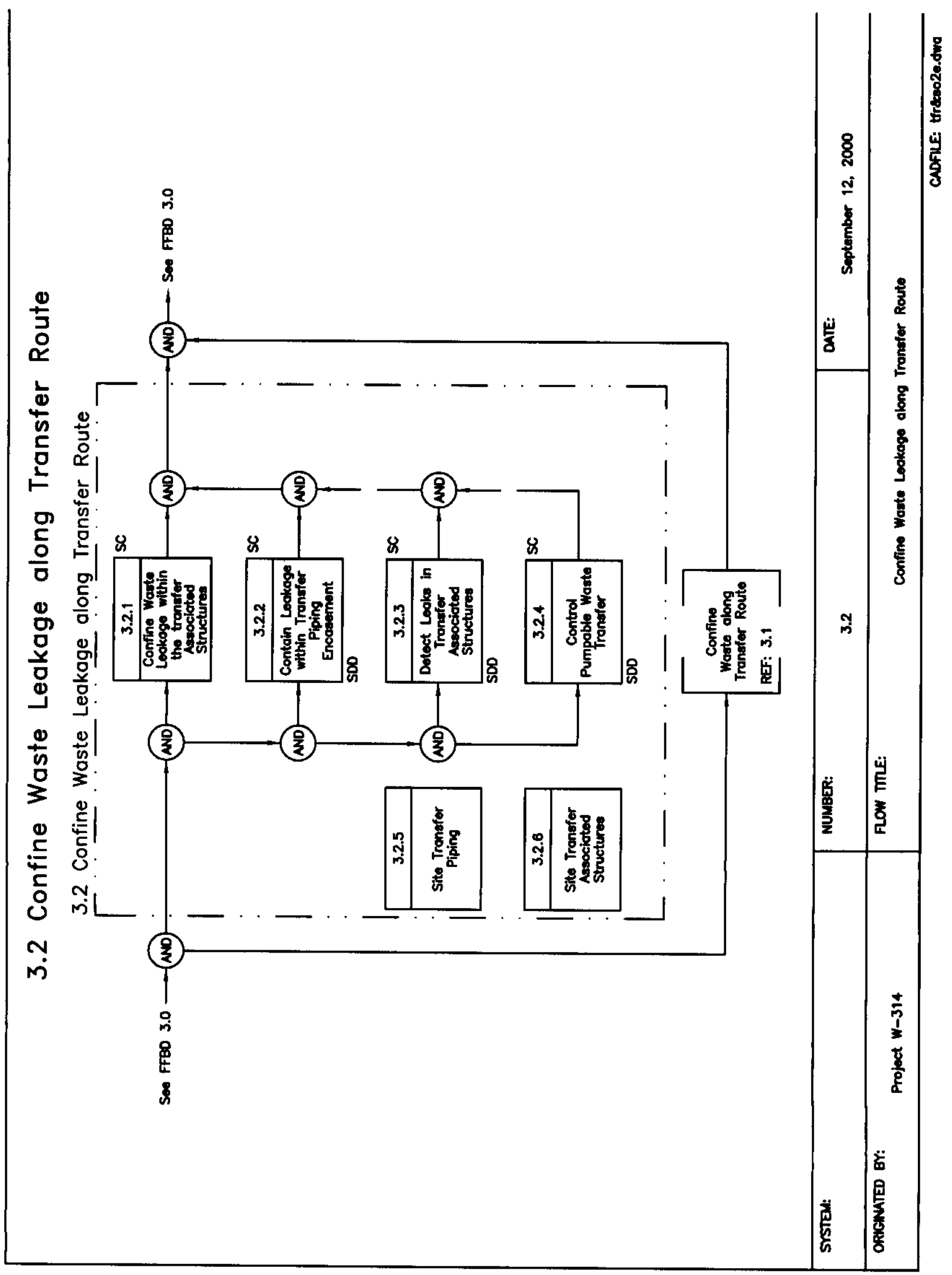


HNF-SD-W314-PDS-002, Rev. 3

APPENDIX B

REQUIREMENTS BASIS

B-1 
HNF-SD-W314-PDS-002, Rev. 3

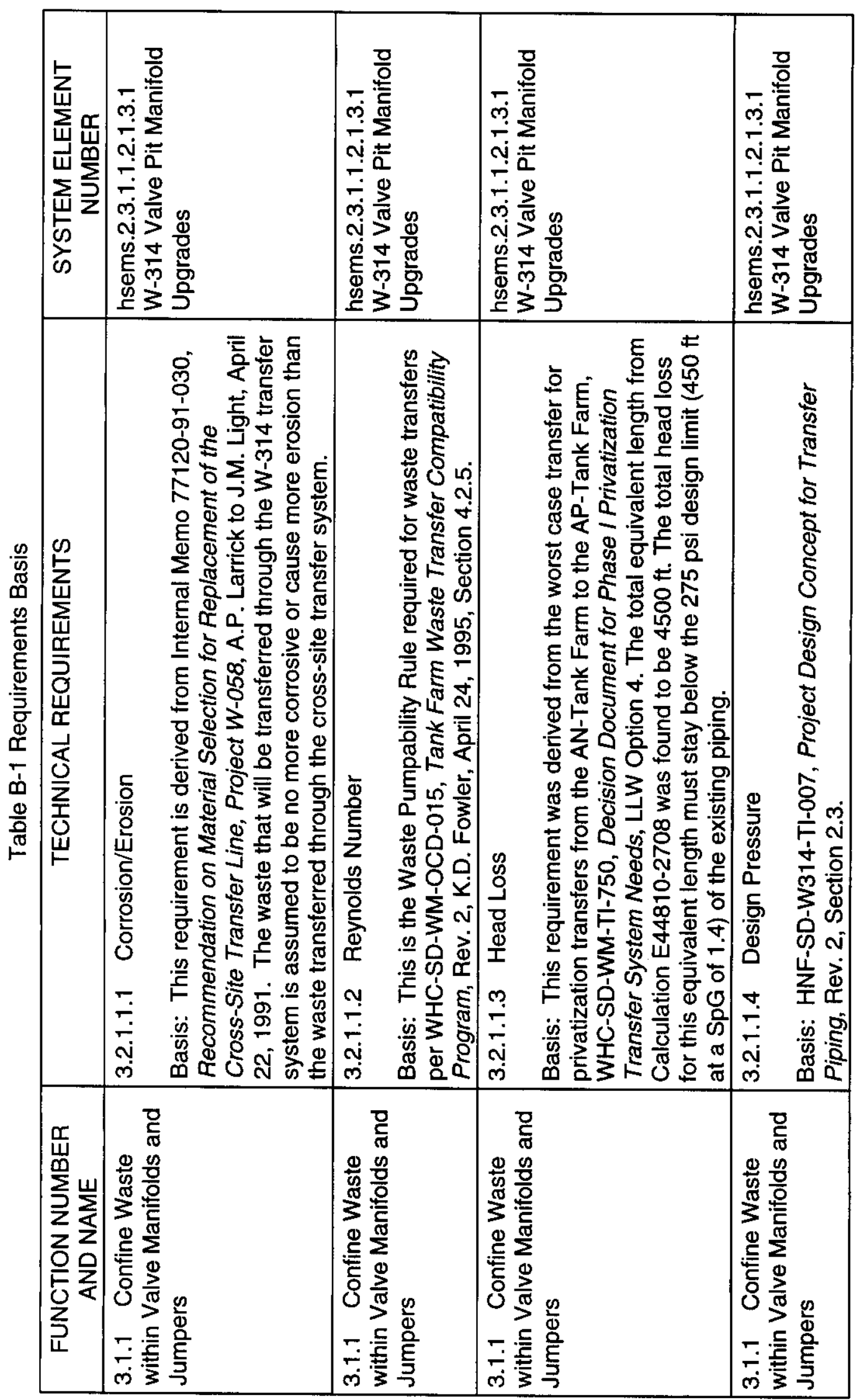




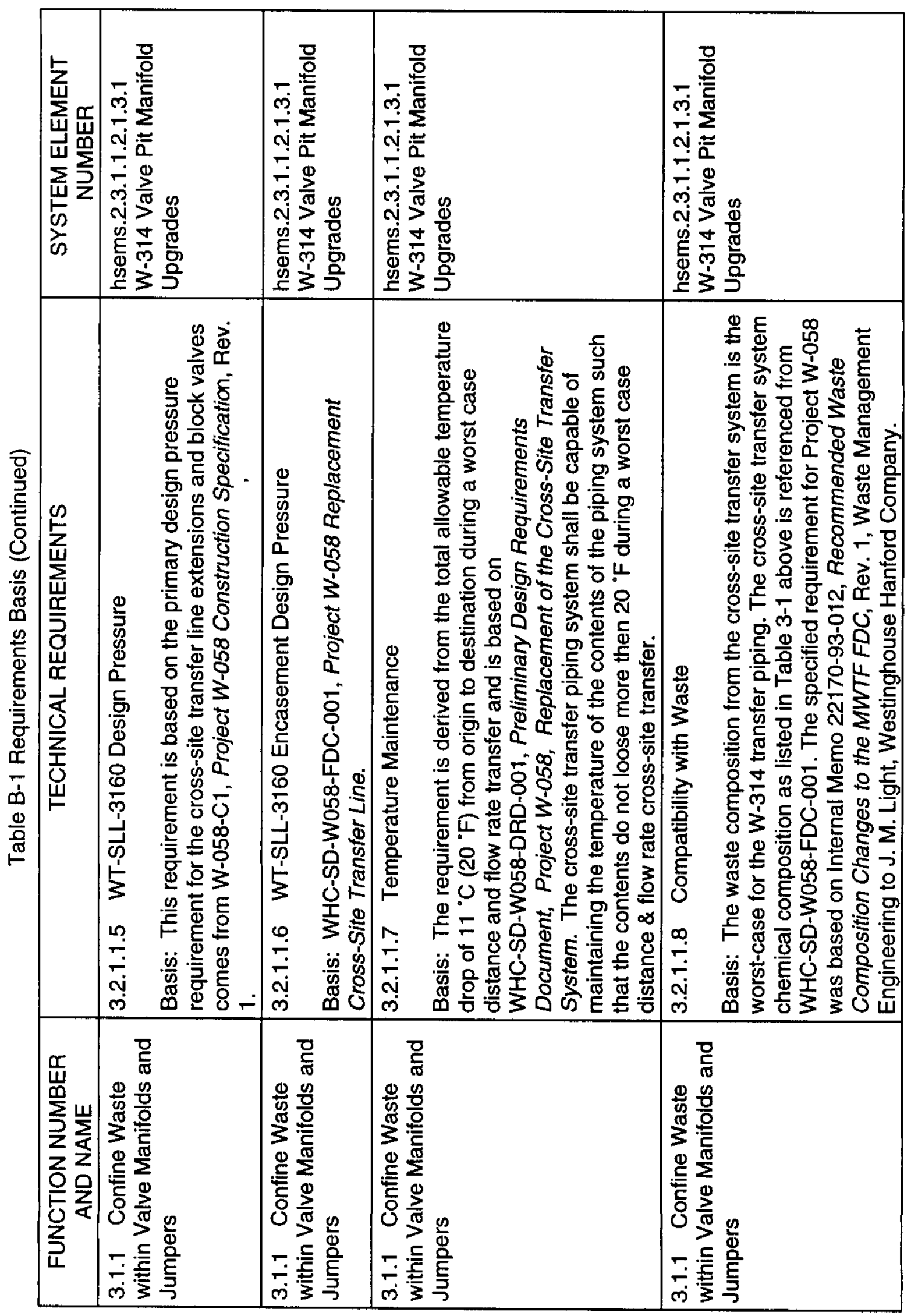


HNF-SD-W314-PDS-002, Rev. 3

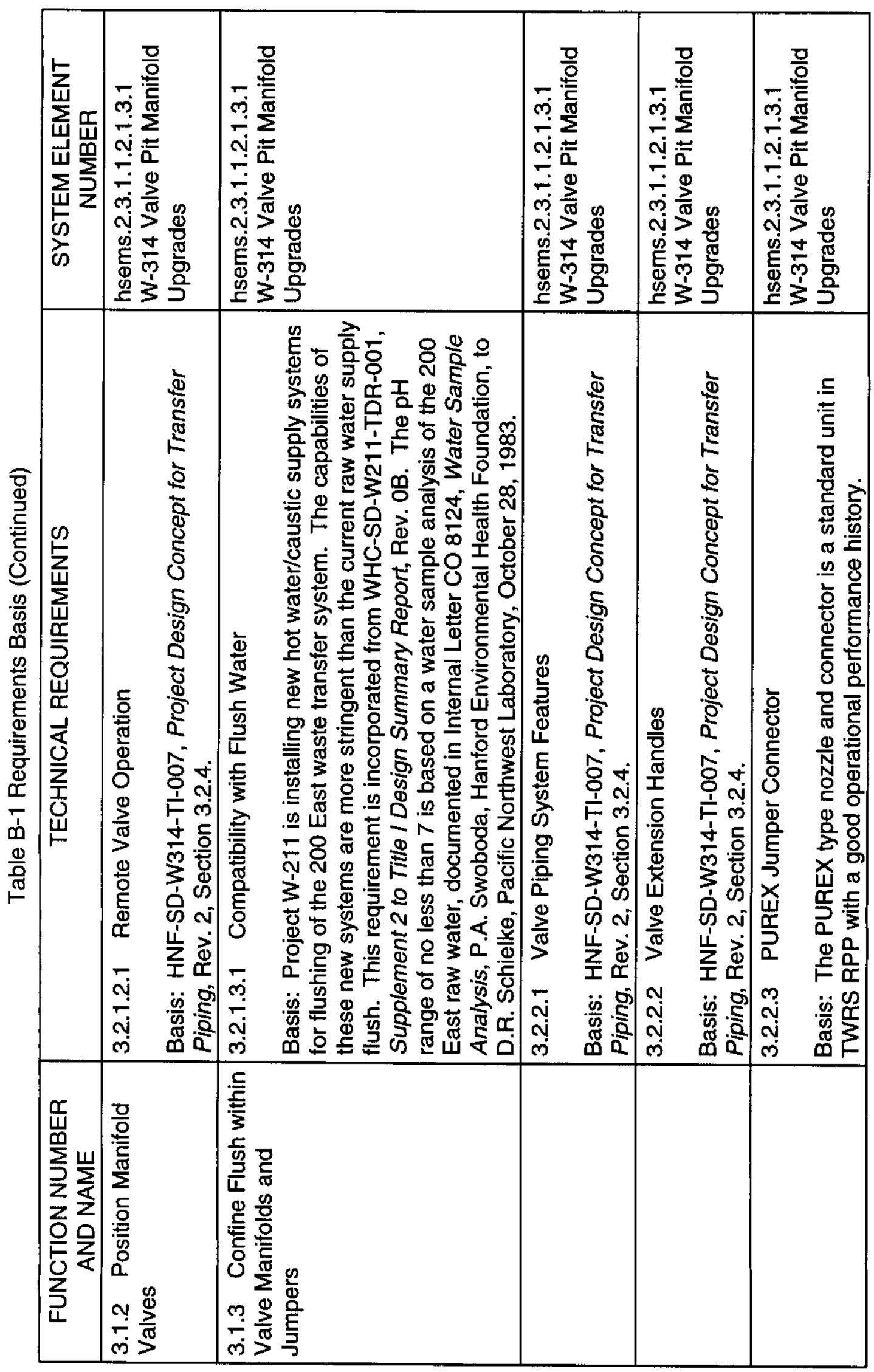


HNF-SD-W314-PDS-002, Rev. 3

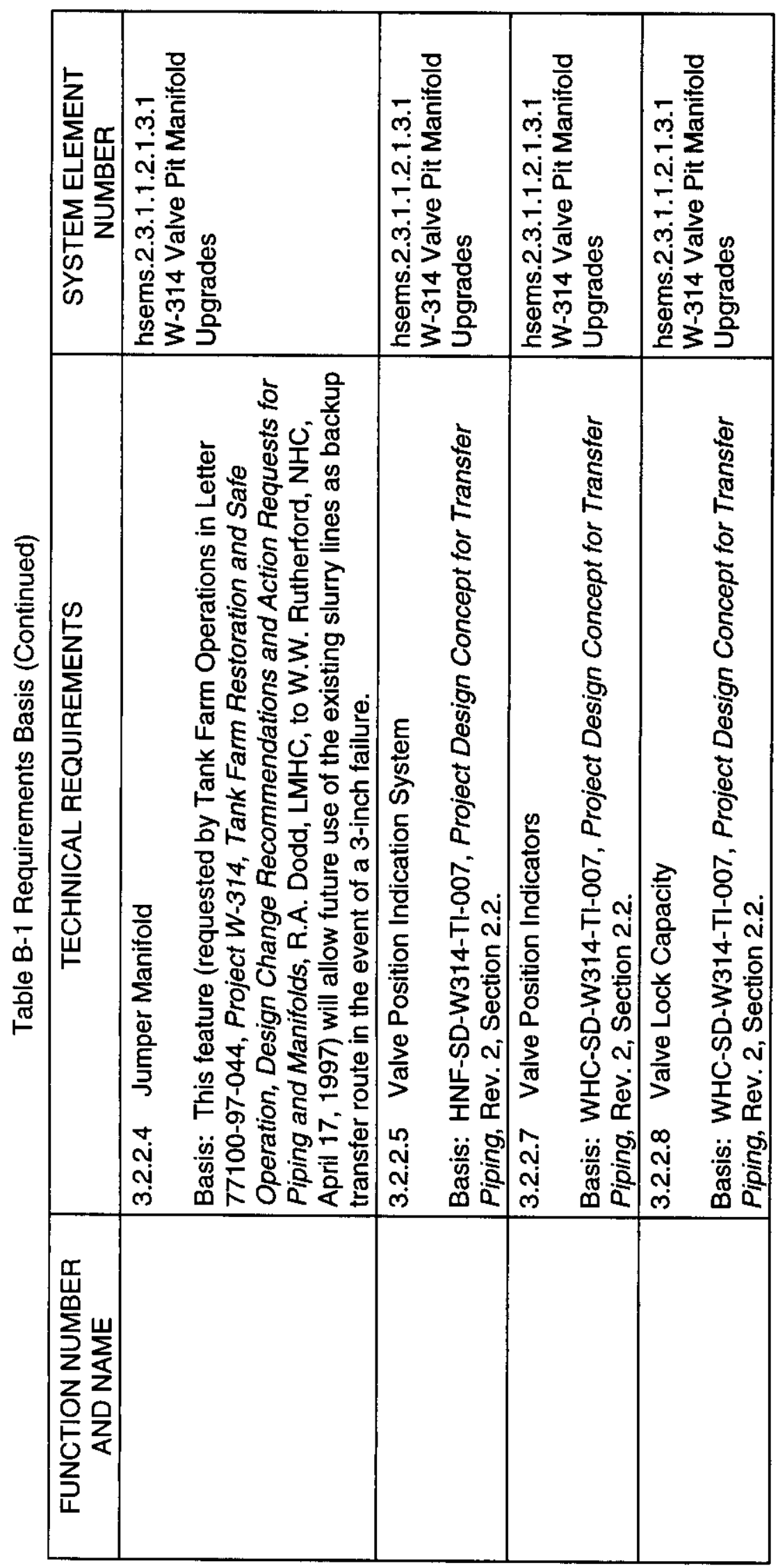


HNF-SD-W314-PDS-002, Rev. 3

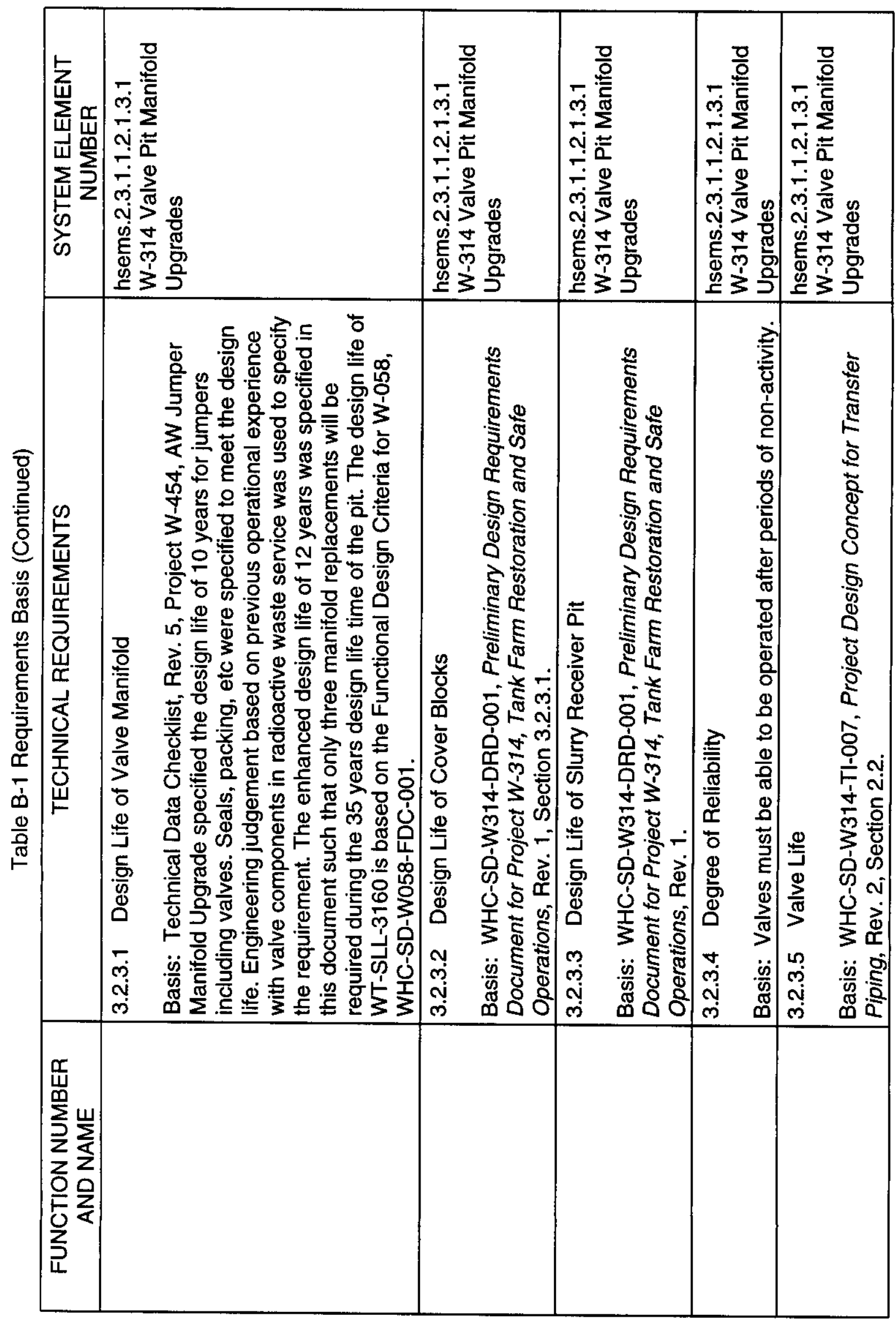


HNF-SD-W314-PDS-002, Rev. 3

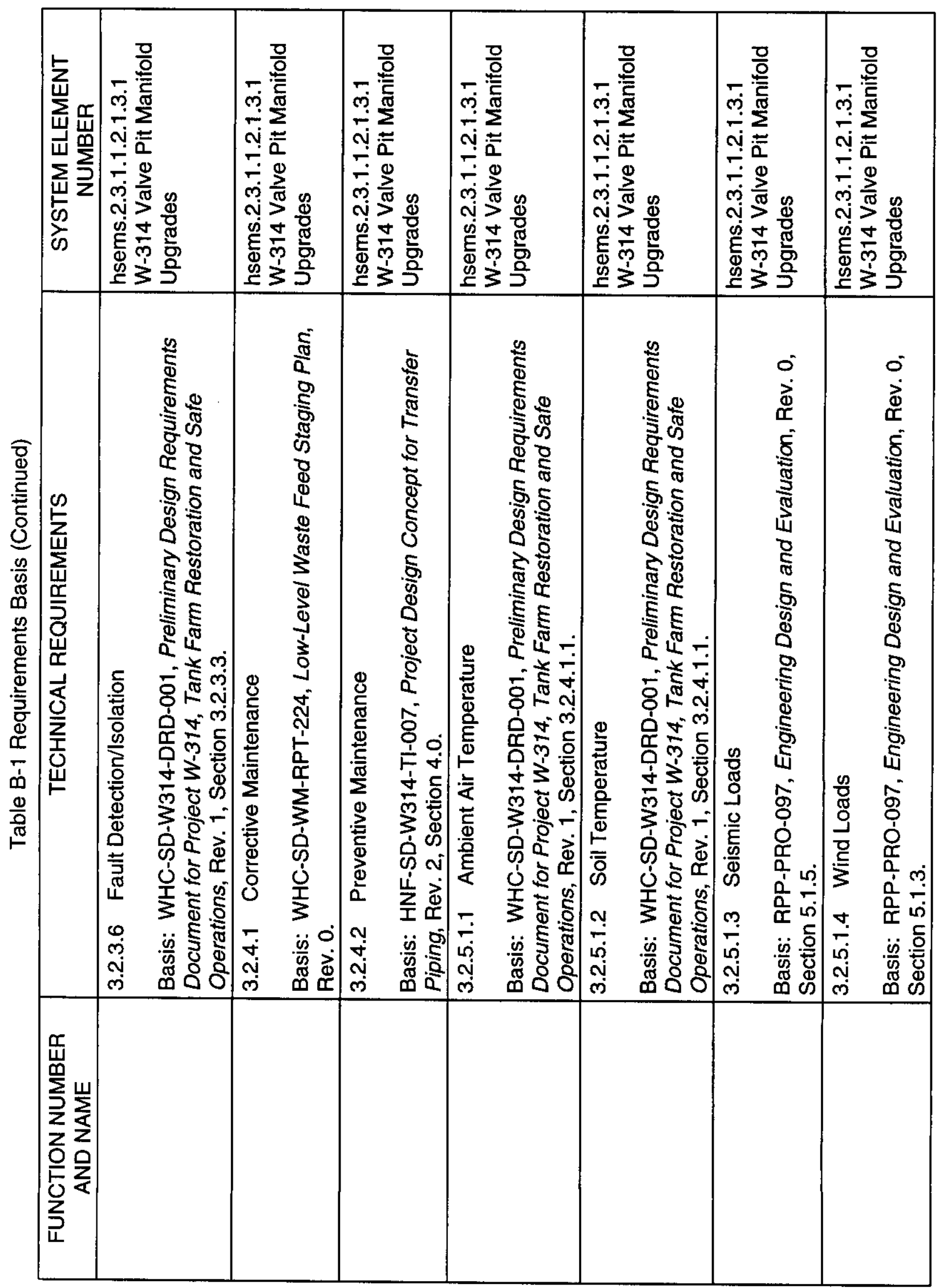


HNF-SD-W314-PDS-002, Rev. 3

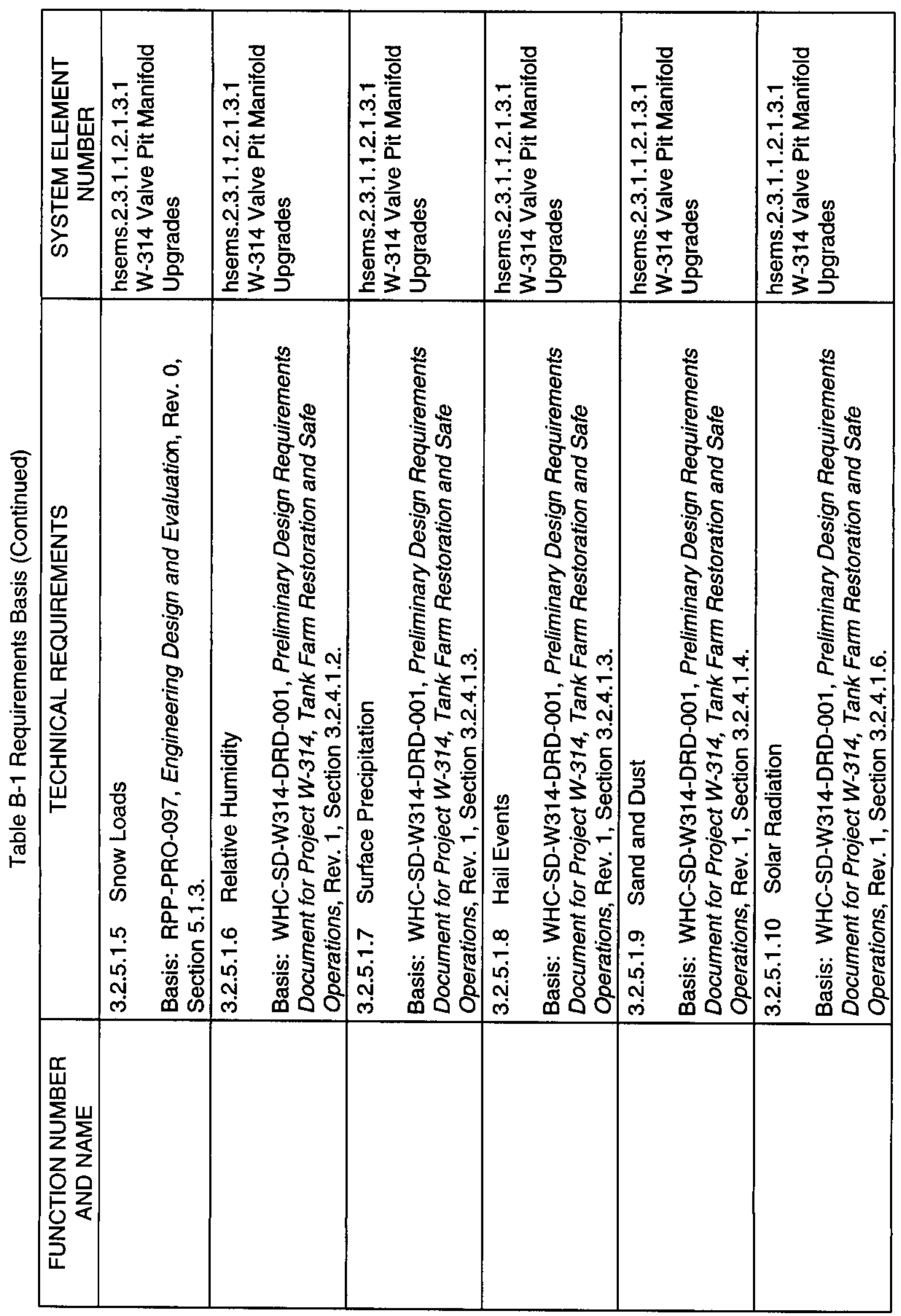


HNF-SD-W314-PDS-002, Rev. 3

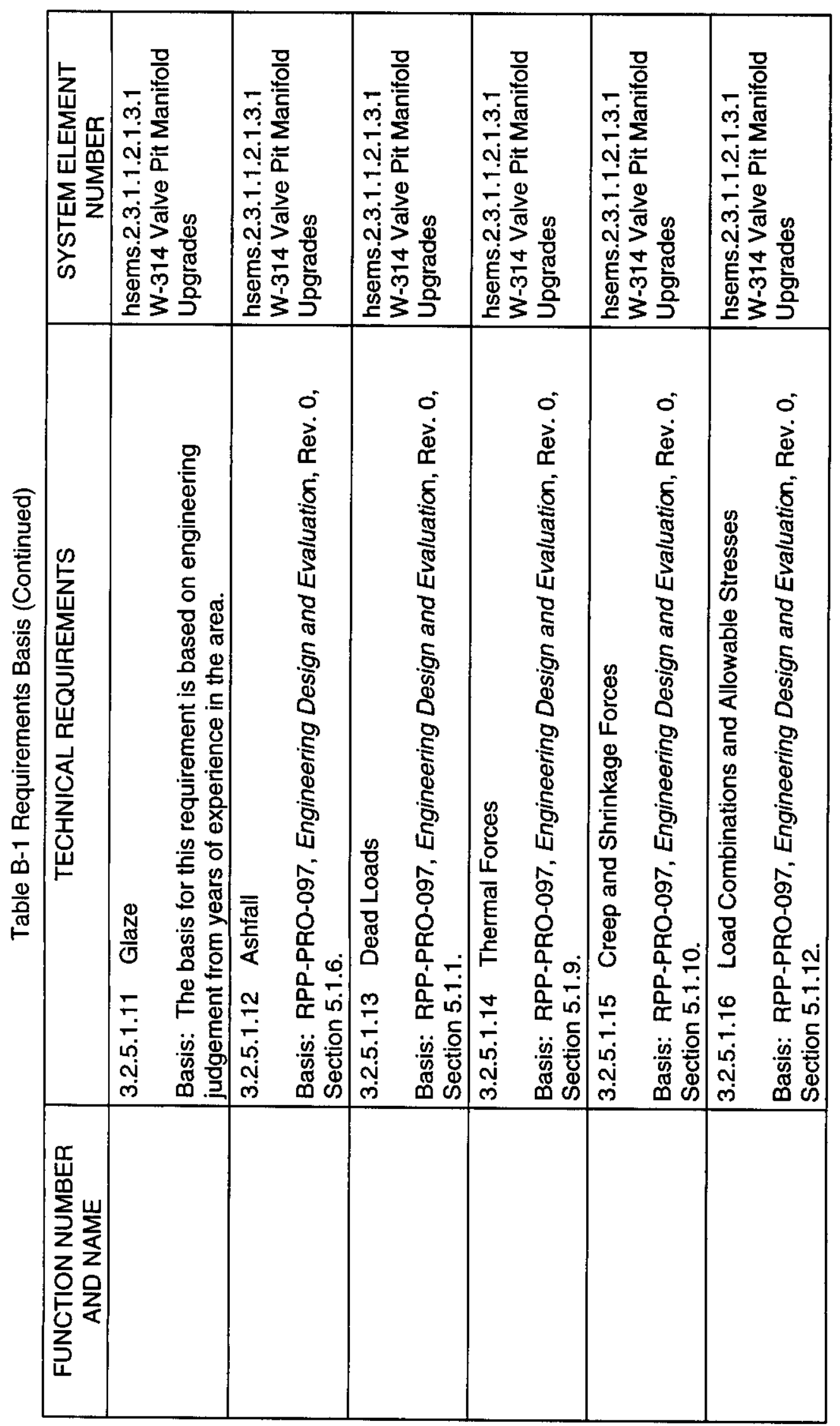




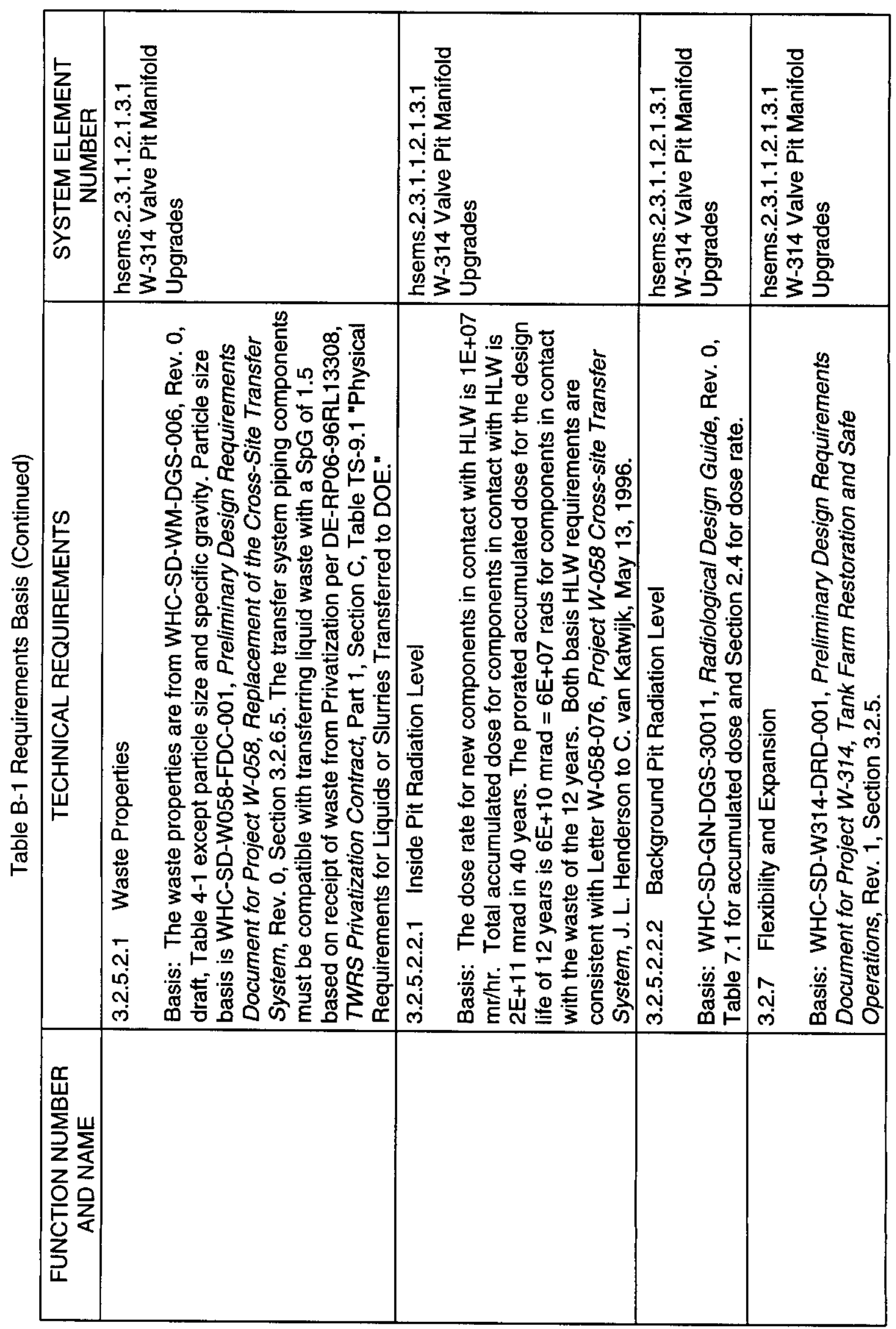


HNF-SD-W314-PDS-002, Rev. 3

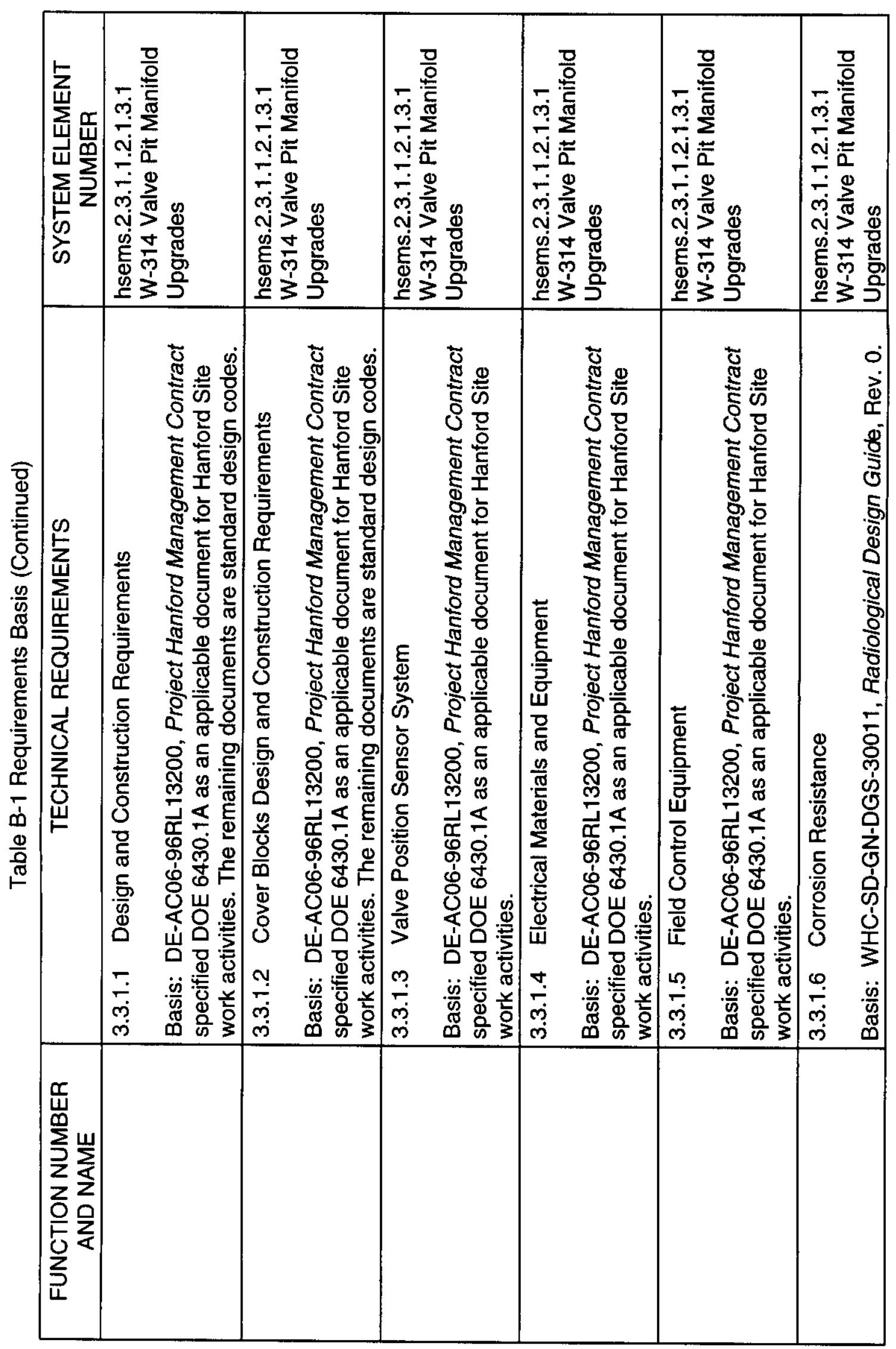


HNF-SD-W314-PDS-002, Rev. 3

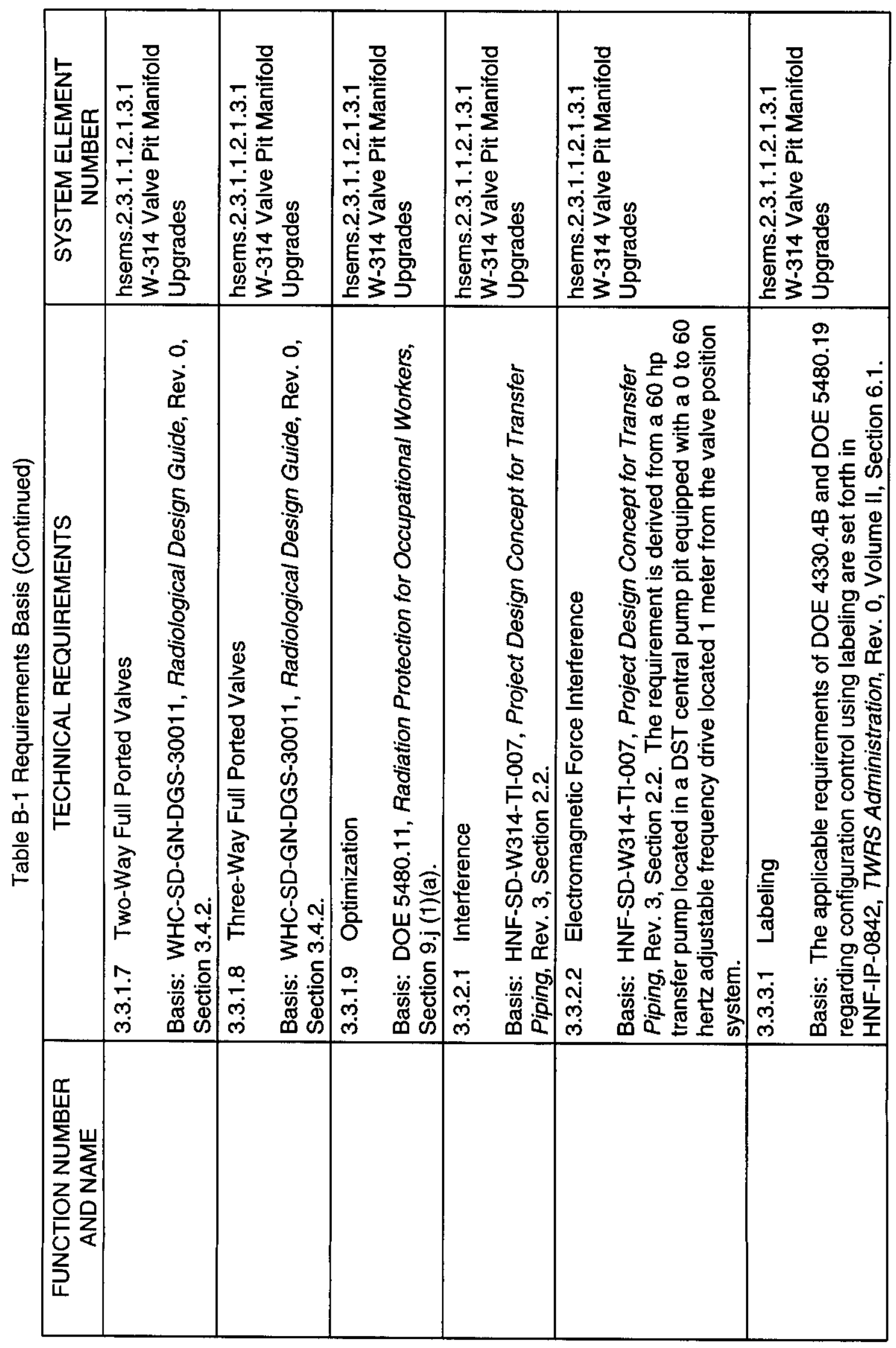


HNF-SD-W314-PDS-002, Rev. 3

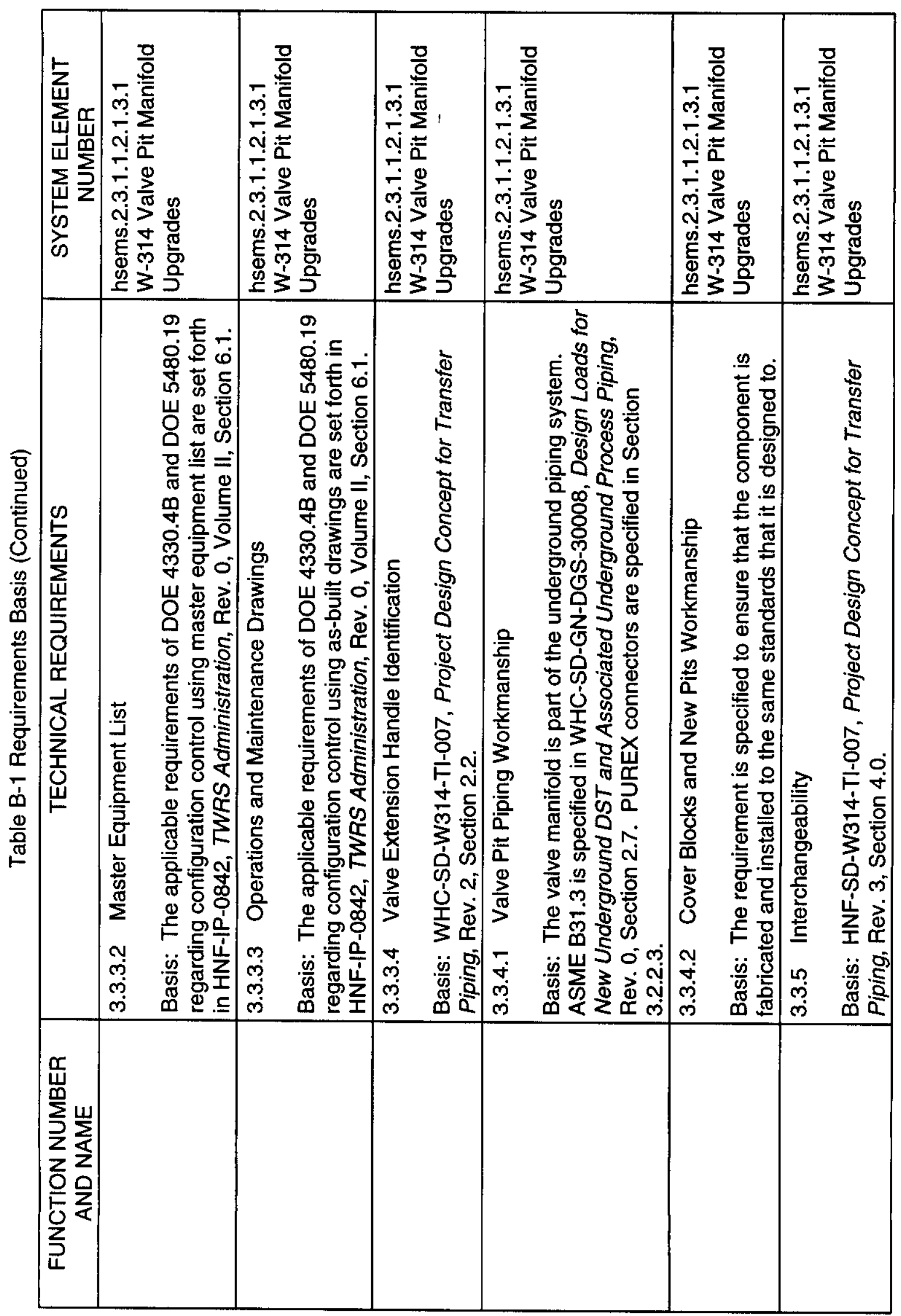


HNF-SD-W314-PDS-002, Rev. 3

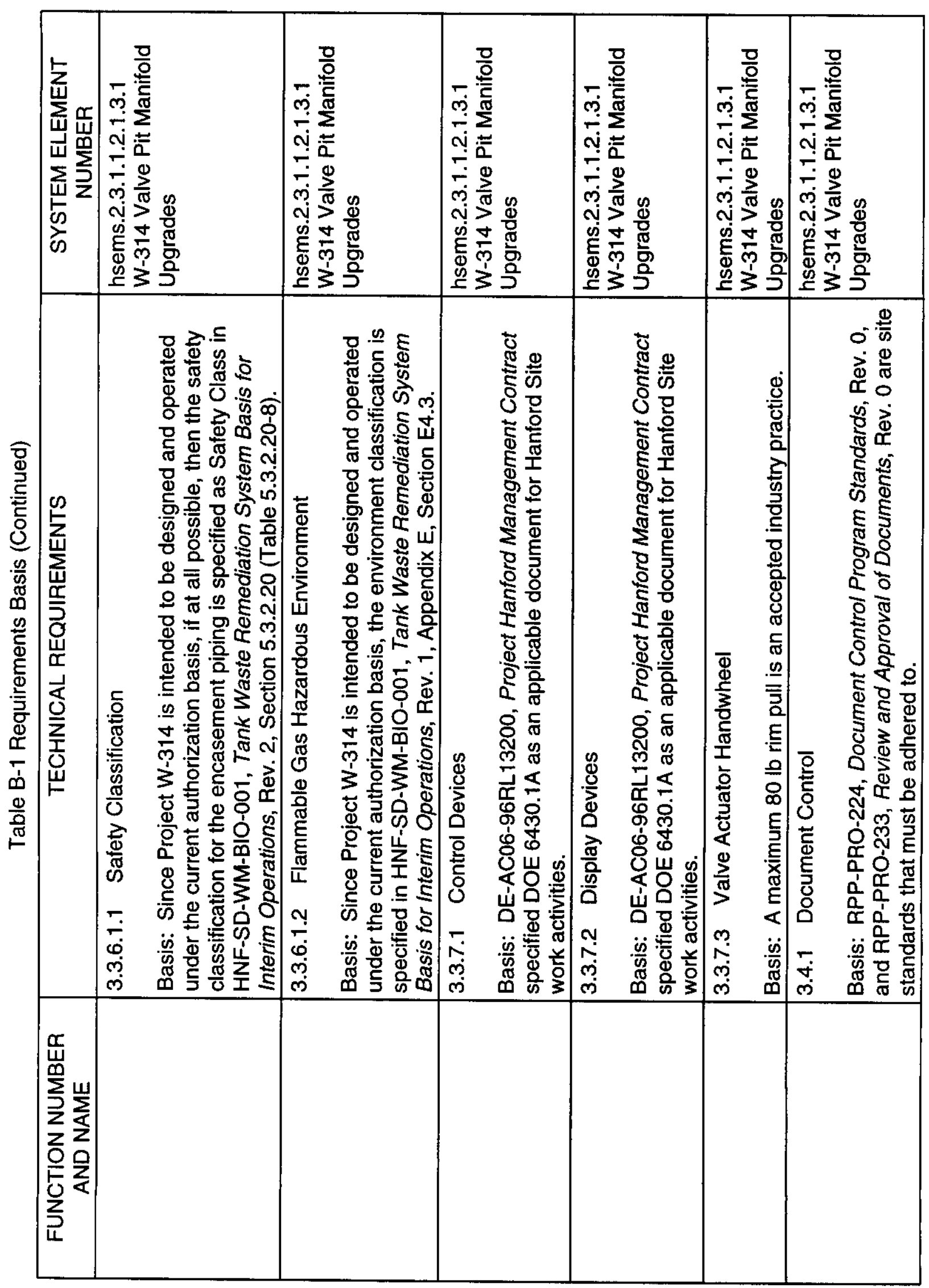


HNF-SD-W314-PDS-002, Rev. 3

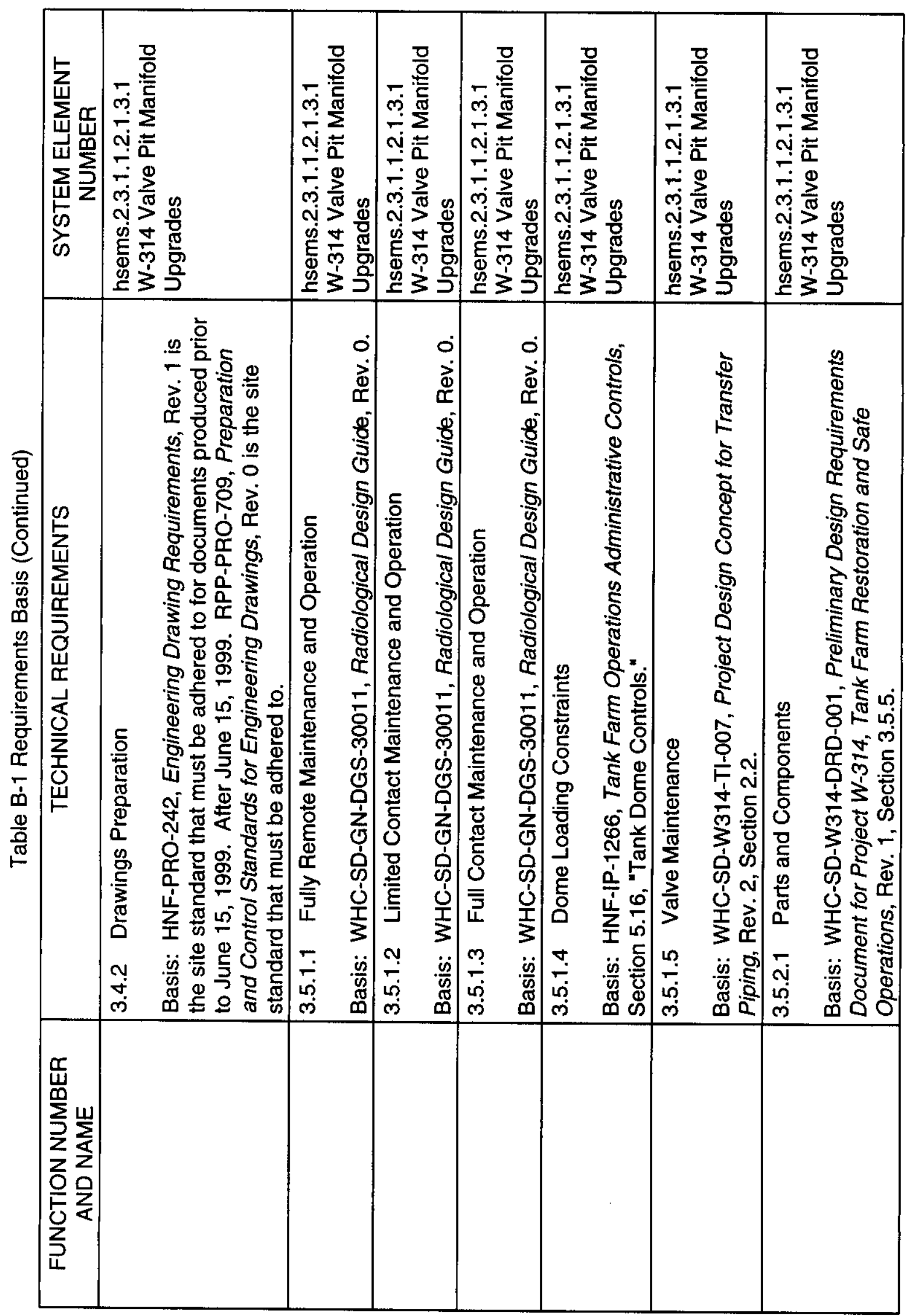


HNF-SD-W314-PDS-002, Rev. 3

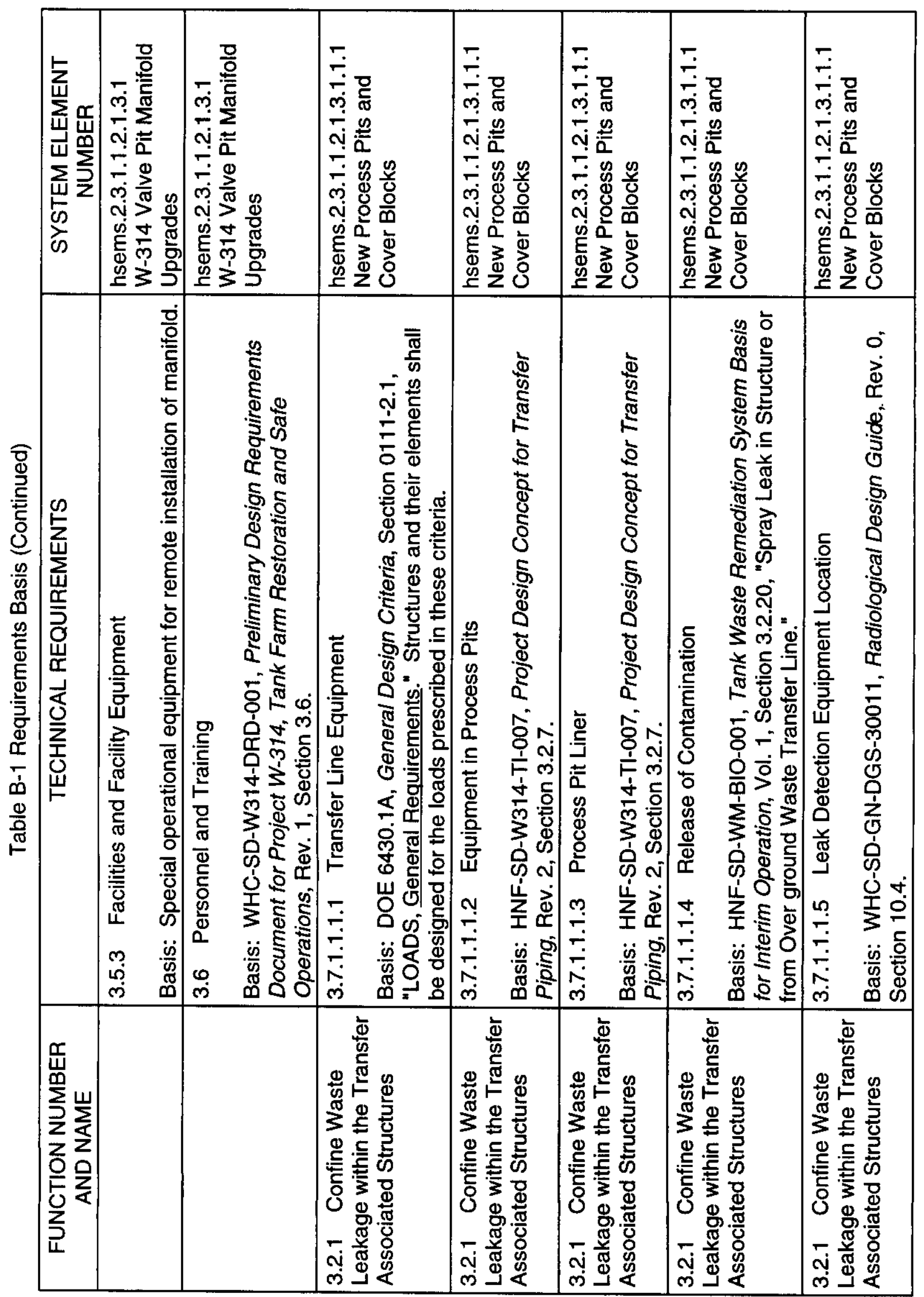


HNF-SD-W314-PDS-002, Rev. 3

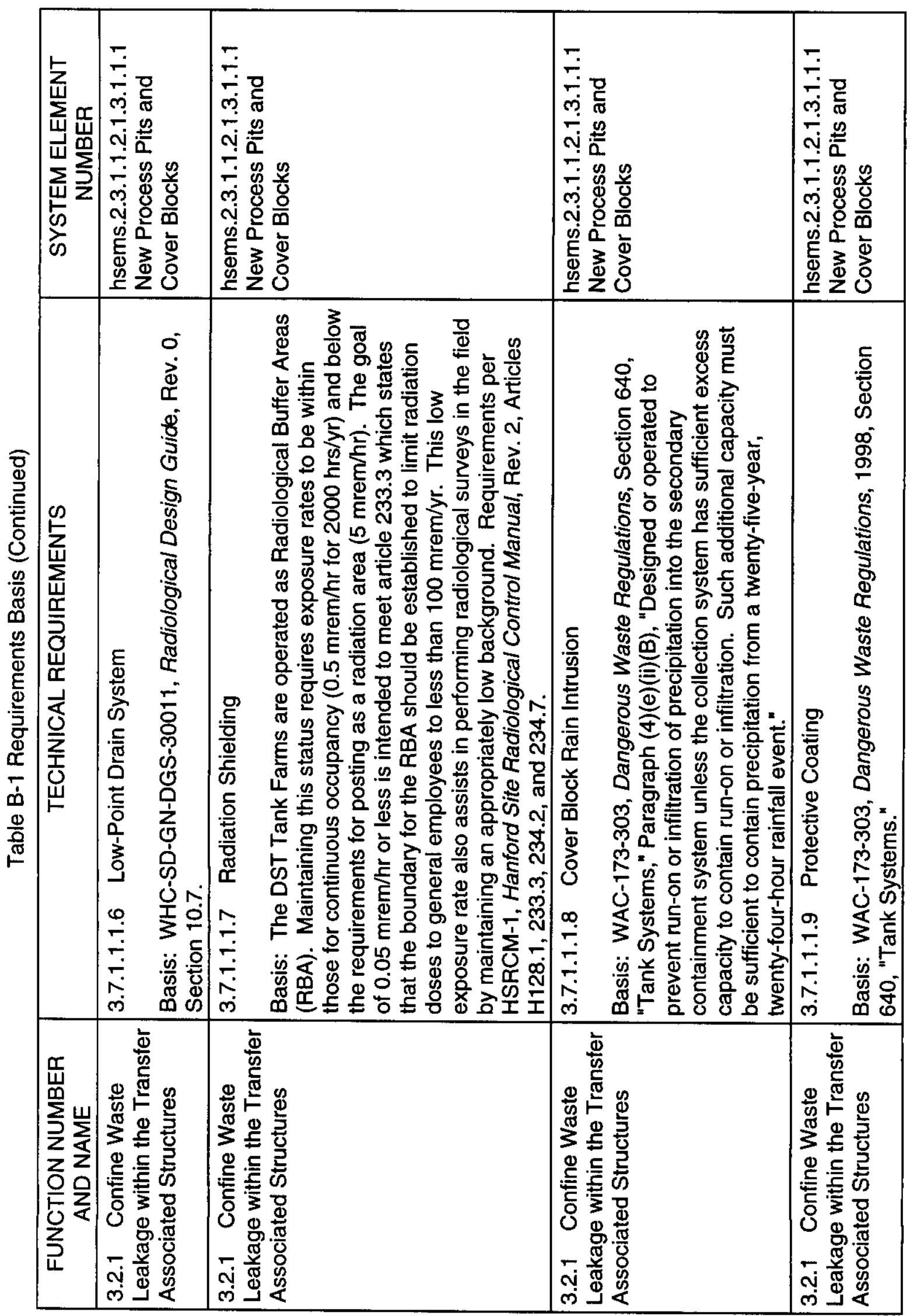


HNF-SD-W314-PDS-002, Rev. 3

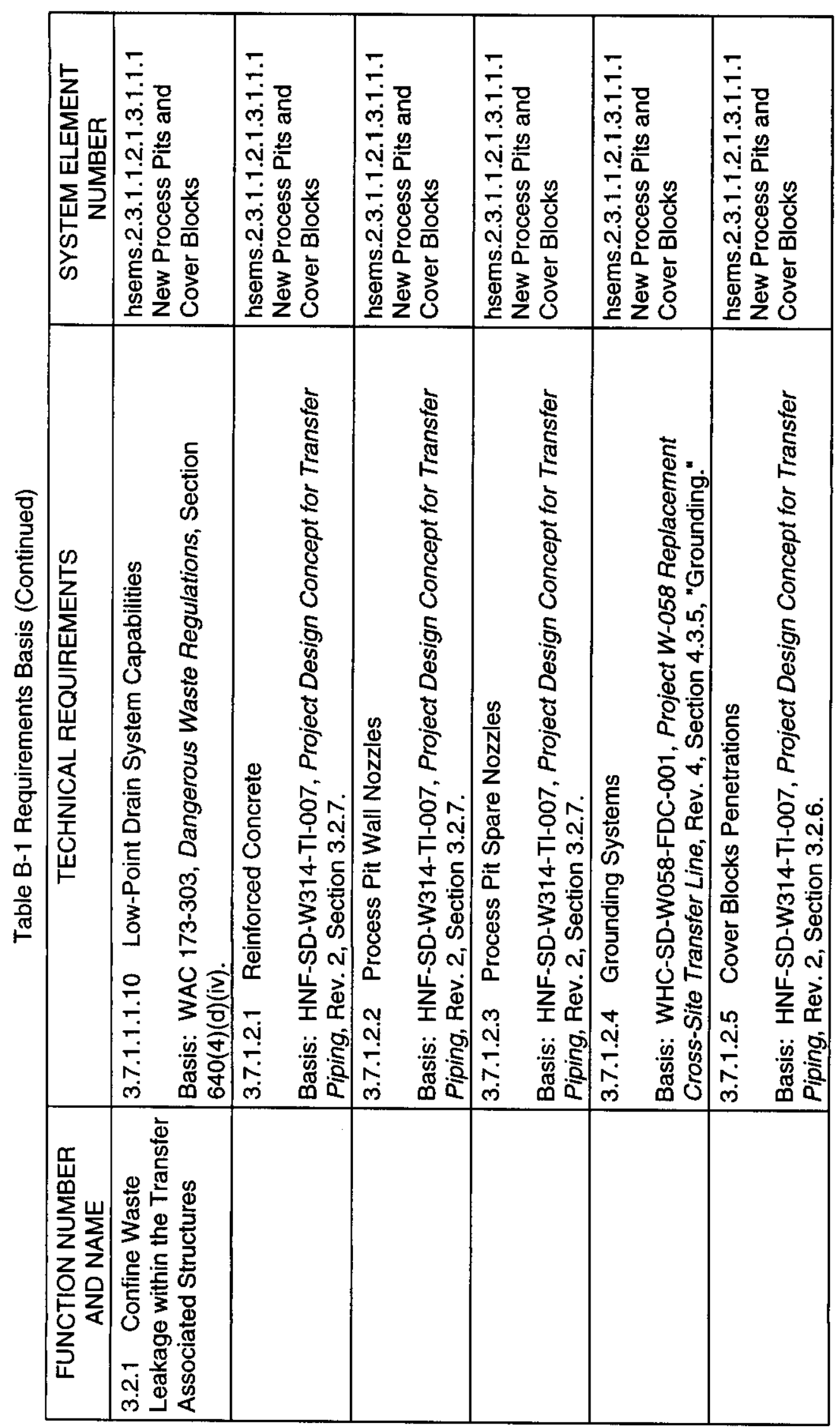


HNF-SD-W314-PDS-002, Rev. 3

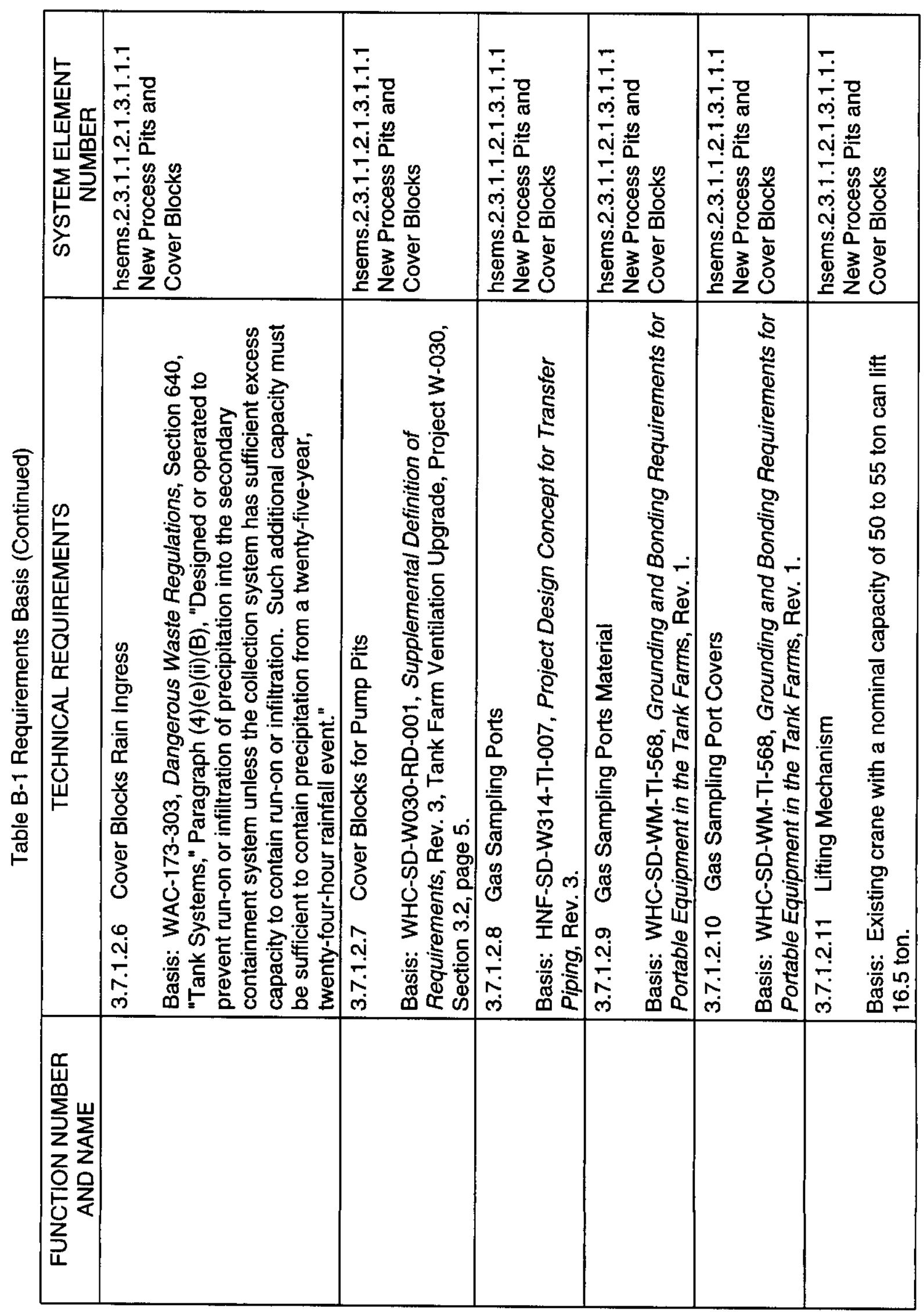


HNF-SD-W314-PDS-002, Rev. 3

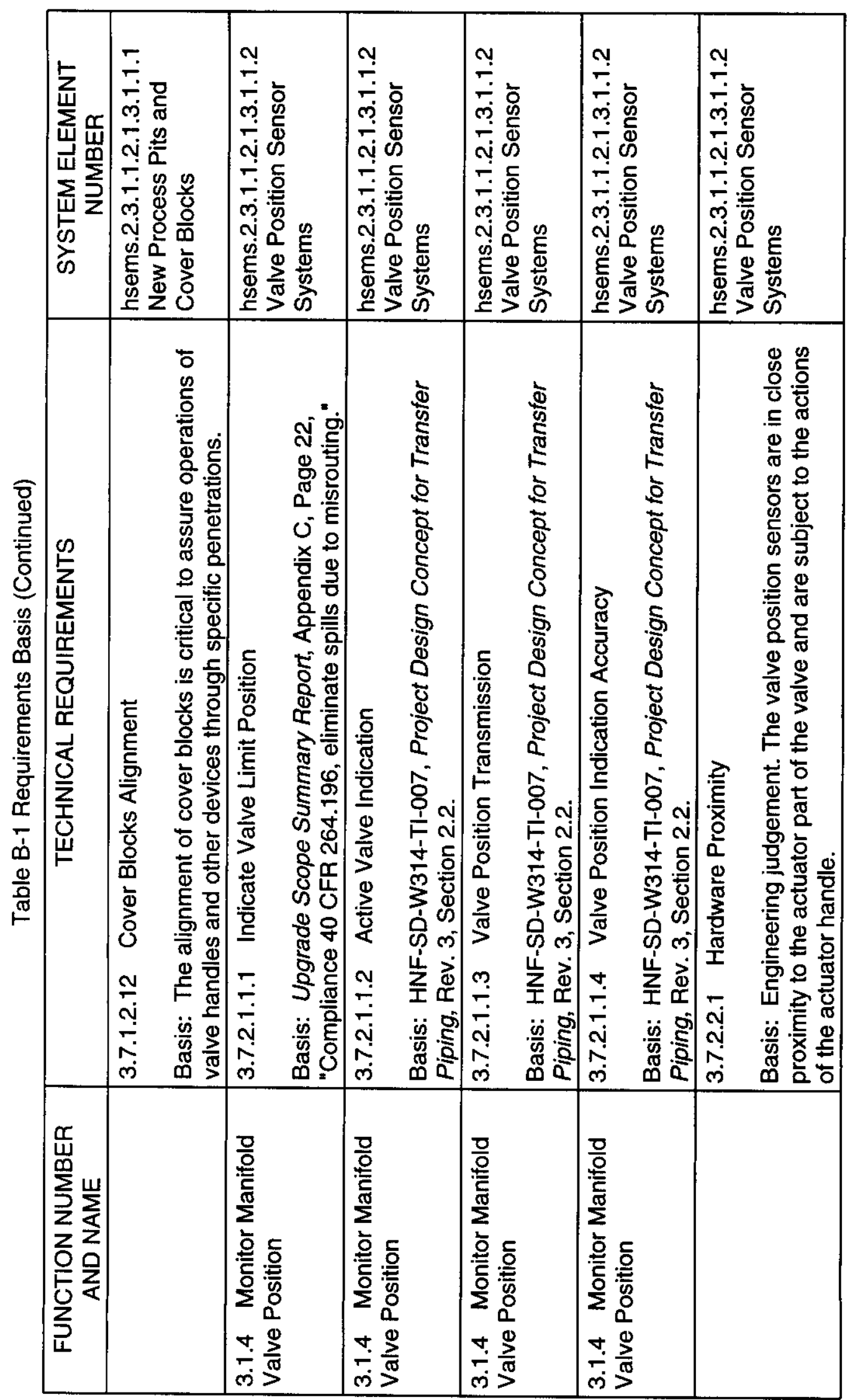


HNF-SD-W314-PDS-002, Rev. 3

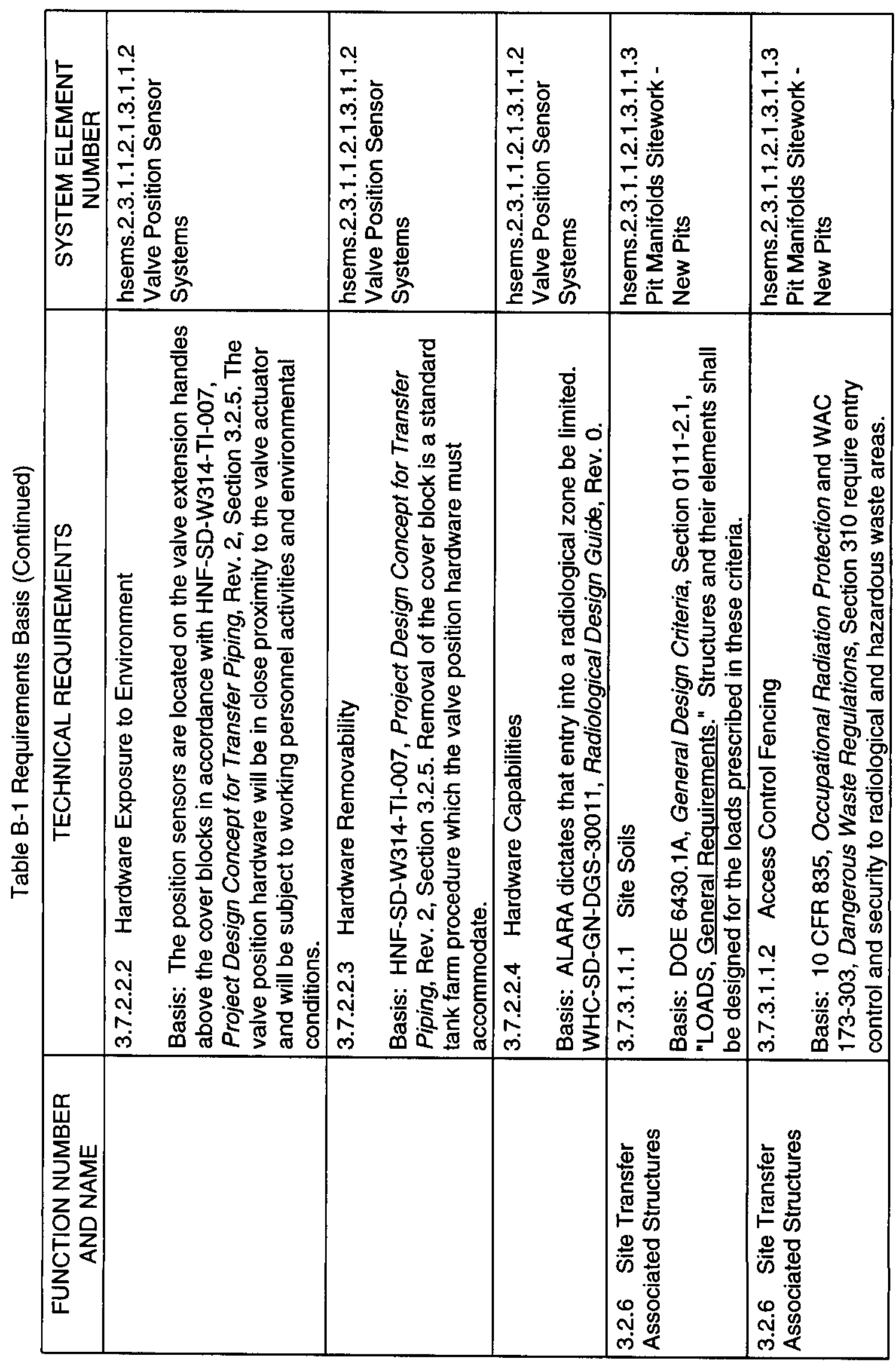


HNF-SD-W314-PDS-002, Rev. 3

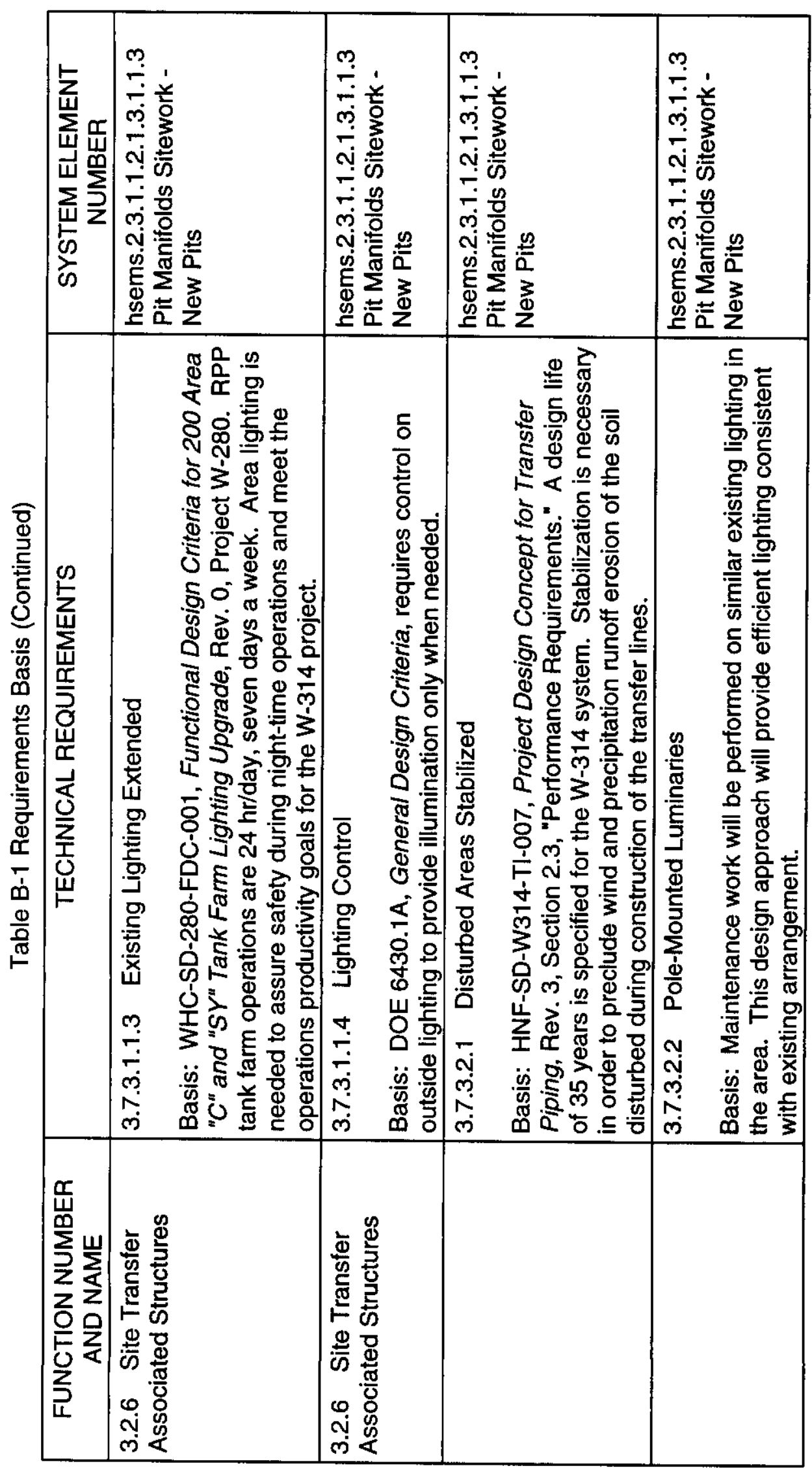


HNF-SD-W314-PDS-002, Rev. 3

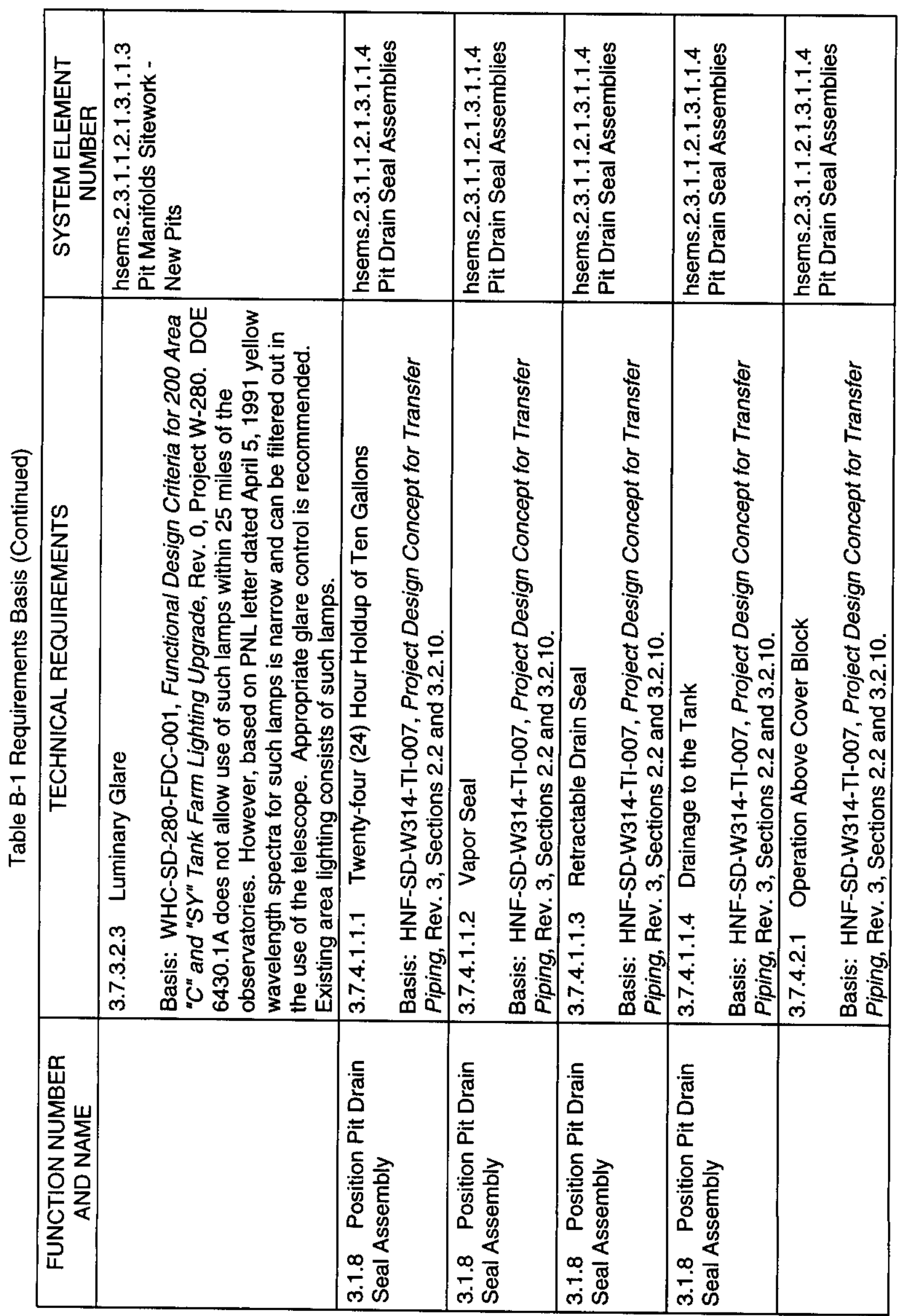


HNF-SD-W314-PDS-002, Rev. 3

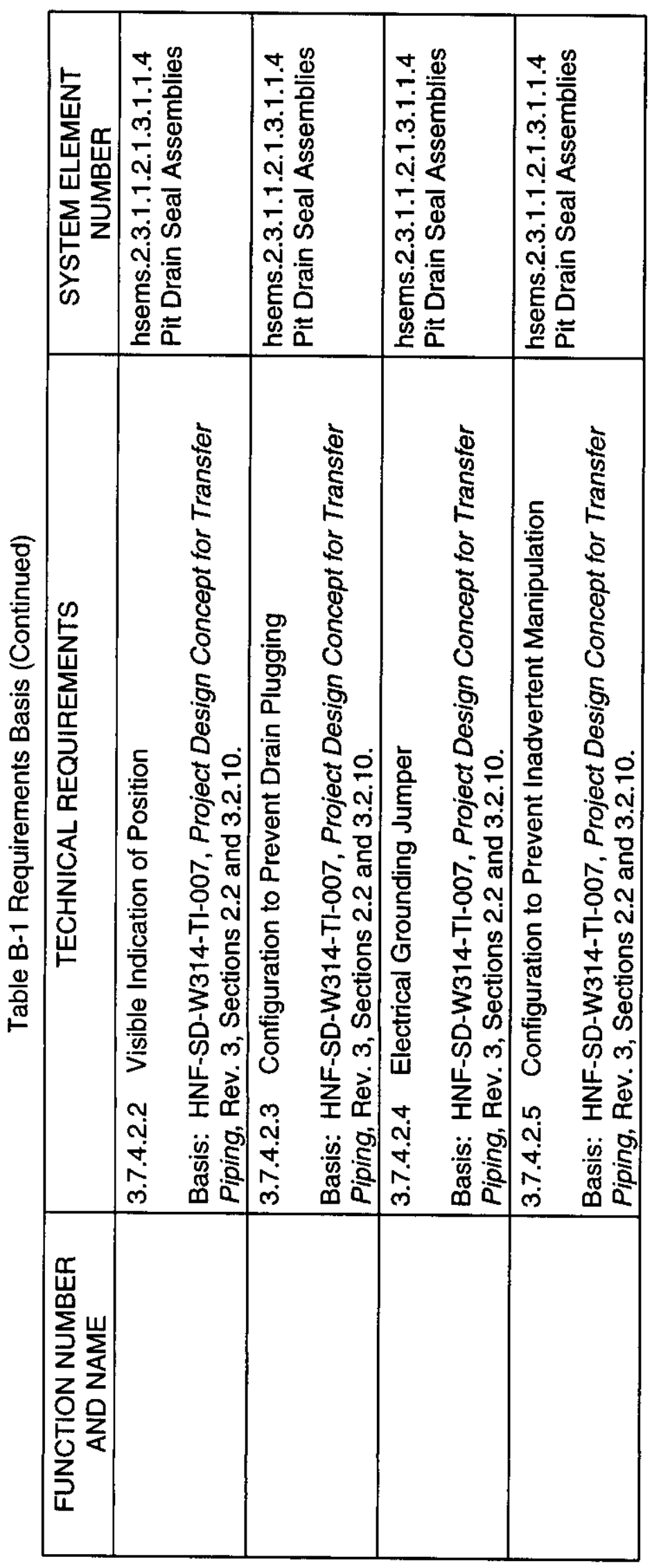

